\title{
Tackling Complexity in Green Contractor Selection for Mega Infrastructure Projects: A Hesitant Fuzzy Linguistic MADM Approach with considering Group Attitudinal Character and Attributes' Interdependency
}

\author{
Junling Zhang $\mathbb{D},{ }^{1}$ Xiaowen $Q i \mathbb{D}^{2},{ }^{2}$ and Changyong Liang ${ }^{3}$ \\ ${ }^{1}$ School of Economics and Management, Zhejiang Normal University, Jinhua 321004, China \\ ${ }^{2}$ School of Business Administration, Zhejiang University of Finance \& Economics, Hangzhou 310018, Zhejiang, China \\ ${ }^{3}$ School of Management, Hefei University of Technology, Hefei 230009, China \\ Correspondence should be addressed to Junling Zhang; zhangjunling@zjnu.cn
}

Received 25 May 2018; Revised 3 October 2018; Accepted 18 October 2018; Published 2 December 2018

Academic Editor: Changzhi Wu

Copyright (c) 2018 Junling Zhang et al. This is an open access article distributed under the Creative Commons Attribution License, which permits unrestricted use, distribution, and reproduction in any medium, provided the original work is properly cited.

Continuous environmental concerns regarding construction industry have been driving general constructors of mega infrastructure projects to incorporate green contractors. Although conventional multiple attributes decision-making (MADM) methodologies have provided feasible ways to select contractor, high complexity in scenarios of megaprojects still challenges existing MADM methods in concurrently accommodating three key issues of decision hesitancy, attributes interdependency, and group attitudinal character. To elicit decision-makers' hesitant fuzzy assessments more objectively and comprehensively, we define an expression tool called interval-valued dual hesitant fuzzy uncertain unbalanced linguistic set (IVDHF_UUBLS) and develop aggregation operators through its operations. To exploit attributes interdependency, we establish a synthesized attributes' weighting model to fuse an attributes interdependency-based weighting vector and an argument-dependent weighting vector, which are, respectively, derived through Decision-Making and Trial Evaluation Laboratory (DEMATEL) technique and maximizing deviation method. To effectively utilize decision-makers' group attitudinal characters, we also develop a TOPSIS-based method to rationally transform group ideal attitudes into order-inducing vectors. On the strength of the above methods, an integrated MADM approach is then constructed. Finally, illustrative case study and experiments are conducted to validate our approach.

\section{Introduction}

In comparison with other industries, the industry of construction has been observed as exerting major contribution to environmental pollution [1], including those related to noise, air, solid waste, and water pollution. $[2,3]$. The problems of construction pollution and wastes have been more critical in countries that are undertaking accelerated urbanization construction with high growth rate of economics. As can be seen, the environmental concerns especially stand out for mega infrastructure construction projects because those megaprojects are basically one-off and sustaining in a relatively very long period $[4,5]$. As a matter of fact, mega infrastructure construction projects are assumed to take responsibility to minimize their negative impact on environments when maximizing contribution to economics and society. To that end, not only high level of project management sophistication is needed but a nexus of green contractors also should be incorporated in project organization [6]. As a result, green contractor selection plays a strategically important role in achieving success of megaprojects.

Due to self-evident complexity in large-scale projects like mega infrastructure, understanding scenarios of work packages and selecting appropriate contractors are intrinsically complicate $[7,8]$. To confront the complexity in contractor selection, Safa et al. [5] suggested utilizing results of competitive intelligence (CI) systems as favorable inputs 
to multiple attributes decision-making (MADM) methodologies. However, during contractor selection activities at the front end planning phase [9], decision-makers' assessments inevitably characterize with vagueness and uncertainty due to limitedness in their experience, knowledge and expertise $[10,11]$. To help decision-makers decrease complexity under different scenarios of green contractor selection, pioneering researches have introduced effective MADM approaches that integrate uncertainty expression tools, such as fuzzy sets [12] and linguistic sets [13-15]. BuiThiNuong et al. [16] put forward a fuzzy AHP method to accommodate contractor selection with imprecise information. Kusi-Sarpong et al. [17] developed a joint rough sets and fuzzy TOPSIS method. Zavadskas et al. [18] presented a weighted aggregated sum product assessment method which uses grey values for contractor selection. When facing more complicate contractor selection problems with ill-structured definition, Vahdani et al. [19] devised an ideal solutions-based MADM approach to contractor selection by employing linguistic variables to describe uncertain assessments. Alhumaidi [20] utilized linguistic variables to construct a fuzzy-logic based multiple attributes group decision-making method. Most recently, in viewing of common phenomena of decision hesitancy in complex decision-making processes [21, 22], Borujeni and Gitinavard [23] studied a group decision-making approach based on hesitant fuzzy preference relations.

Actually, advocating practicality of hesitant fuzzy set (HFS) [21], one of popular research directions in fuzzy MADM literature has also focused on tackling complicate situations with decision hesitancy based on HFS [40]. Such as the MADM approach based on Shapley-Choquet integral operators to consider interdependencies among attributes [41], the hesitant fuzzy TOPSIS method [42], the MADM approach based on hesitant fuzzy Hamacher aggregation [43], the hesitant fuzzy QUALIFLEX approach [44], among others. Following the idea of HFS, Zhu et al. [45] extended HFS to the dual hesitant fuzzy set (DHFS) by adding nonmembership degrees in depicting hesitancy. Since then, DHFS has been studied with a deep extent, such as those listed in [4650]. Regarding decision-making situations where linguistic variables gain feasibility and adaptability, on the basic ideas of HFS, Rodríguez et al. [51] firstly introduced the hesitant fuzzy linguistic term sets to allow decision hesitancy of possible linguistic terms. However, in practical cases, decision-makers are capable of reaching the most favorite linguistic label but generally holding decision hesitancy to that label; or under the decision-making situations where voting and majority rule apply, group opinions will locate at a linguistic label or a linguistic interval [52] but obviously there exists decision hesitancy to the voted label. As a result, compound expression tools of hesitant fuzzy linguistic sets and their MADM approaches have attracted widespread interests. Such as, hesitant fuzzy linguistic set $[38,53]$ and dual hesitant fuzzy linguistic set $[39,54]$, among others. Comparatively, compound hesitant fuzzy linguistic expression tools attain more adaptability and feasibility in practical complex decision environments.

Although researches in both contractor selection literature and MADM literature have presented rich approaches for application to green contractor selection problems, there is still a gap with three facets to be further investigated concurrently. First, most of existing hesitant fuzzy linguistic expression tools were based on rigidly uniform or symmetrical linguistic label sets $[55,56]$; however, practical studies $[57,58]$ have revealed that decision-makers are inclined to express their complicate assessments more precisely and objectively by use of non-uniform or asymmetric linguistic term set, that is, the unbalanced linguistic term set (ULTS) [59]. Second, under complicate decision-making environments, interdependency among attributes is a serious issue that needs to be addressed [60]. However, Choquet integral incorporated by Lin et al. [38] can only capture interactions between adjacent coalitions, while the prioritized aggregation operator in $[53,54]$ requires linear ordering of evaluative attributes that is often difficult for decision-makers to deduce up front in complex practices. Third, in order to deal with real-world complex problems, various degrees of optimism (degree of orness) of decision-makers should be coordinated as complex attitudinal characters and taken into account during decision-making [61]. Merigó and Casanovas [61] suggested to use order-inducing variables for reflecting complex attitudinal characters and integrate them into information aggregation operators, in which the order-inducing variables are usually not directly defined and need to be derived appropriately [62]. But to our best knowledge, thus far, there is scarcely any attention has been paid to the complex attitudinal characters when tackling complicate contractor selection problems.

Therefore, to bridge the above-discussed gap, we first introduce an interval-valued dual hesitant fuzzy uncertain unbalanced linguistic set (IVDHF_UUBLS) whose elements hold a hybrid structure of $\langle x, \widetilde{s}, \widetilde{h}(x), \widetilde{g}(x)\rangle$, in which two interval-valued fuzzy sets $\widetilde{h}(x)$ and $\widetilde{g}(x)$ denote possible membership and nonmembership degrees to the uncertain unbalanced linguistic term $\widetilde{s}$. IVDHF_UUBLS can depict fuzzy properties of an object more comprehensively and flexibly. We also develop a series of generalized aggregation operators for IVDHF_UUBLS. In order to exploit interdependency relations among evaluative attributes more effectively, we next employ the Decision-Making and Trial Evaluation Laboratory (DEMATEL) $[63,64]$ to capture both direct and indirect influences between attributes, thereby constructing a more rational attributes weighting model. To include decision-maker's complex attitudinal characters, instead of assuming subjective values, we propose a robust TOPSISbased method to identify the order-inducing variables based on positive and negative ideal attitudes. On the strength of above methods, we subsequently propose an effective MADM approach for resolving the complex problems of green contractor selection.

The rest of this paper is organized as follows. In Section 2, research problem is described. Section 3 defines the new hybrid hesitant fuzzy linguistic expression tool, i.e., IVDHF_UUBLS, and studies basic operations and distance measure for IVDHF_UUBLS. Section 4 investigates several fundamental generalized aggregation operators for 
IVDHF_UUBLS. In Section 5, We detail the DEMATELbased model for weighting evaluative attributes and the TOPSIS-based method for deriving order-inducing variables, based on which we then construct an effective approach for complex MADM. In Section 6, illustrative case study and experiments are further conducted to verify the proposed MADM approach. Finally, conclusions and future research are presented in Section 7.

\section{Description of Research Problem}

The significant impacts of construction activities on the environment have triggered serious alarms and governments worldwide have introduced various policies, regulations and industrial evaluation systems for controlling them [65], for instance, the cleaner production promotion law and the pollution prevention law in China and the green building rating systems in the US, UK, and China [66]. To meet the requirements of environmental concerns, construction projects have to select contractors in operations by simultaneously considering potential contractors' green characteristics [6]. Actually, the practical needs of selecting green contractors are especially indispensable for mega infrastructure construction projects as they generally are one-off and nearly ever-lasting in the environment.

2.1. Evaluative Attributes for Green Contractor Selection. Typically, when construction organizations seek to develop appropriate approaches to contractor selection, the organizations' specific requirements are firstly introduced. Therefore, different sets of evaluative attributes for contractor selection with different scenarios are needed. During last two decades, many efforts have been paid to identify selection attributes and construct comprehensive contractor selection methods with different applications $[8,10,18,19,23,24,26,28,67,68]$.

One basic observation from existing literature for considering evaluative attributes is that, being analogous to selecting suppliers in supply chain management [69], decision-makers are supposed to not only consider competitive capability that distinguishes contractors from each other by business operations, but also examine contractor's cooperation capacity with other partner contractors that indeed influences the project success $[8,70]$. More importantly, since the construction industry has great impact on environment and the governments worldwide have introduced various policies and regulations for controlling them [6], green practices thus has become a crucial facet for evaluating green contractors $[68,71]$. As a result, three aspects of business competiveness, cooperation, and green practices thus become indispensible to derive evaluative attributes for green contractor selection.

Many earlier studies put their emphasis on competitive attributes to evaluate comprehensive performance of contractors. Hatush and Skitmore [26] suggested five competitive attributes to assess contractors, including financial soundness, technical ability, management capability, health and safety, and reputation. Fong and Choi [24] studied contractor selection problem for Hong Kong scenario and derived a set of eight evaluative attributes according to a questionnaire survey, among which seven are competitive attributes including price, financial capability, past performance, past experience, resource, current workload, and safety performance. In their developed computer-aided decision support system, Shen et al. [28] employed a parameter system to evaluate contractor's competitiveness in the Chinese construction industry, in which six competitive attributes were included as social influence, technical ability, financing ability and accounting status, marketing ability, management skills, and organizational structure and operations. Darvish et al. [25] developed a graph theory-based decision-making method to cope with contractor prequalification problem under Iranian scenarios; they introduced the Iranian domestic prequalification criteria system that included nine main indicators: work experience, technology \& equipments, management, experience and knowledge of the operation team, financial stability, quality, being familiar with the area or being domestic, reputation, and creativity and innovation. NietoMorote and Ruz-Vila [10] also investigated a fuzzy decisionmaking model based on TOPSIS to accommodate construction contractor prequalification problem; they summarized the most common factors for comprehensively considering contractors during the prequalification that fall into following main aspects: technical capacity, experience, management capability, financial stability, past performance, past relationship, reputation, and occupational health \& safety. Focusing on contractor selection problems in Lithuania construction industry, Zavadskas et al. [18] proposed an effective multiple attributes decision-making approach called WASPAS-G, in which main attributes are identified to cover: bid amount, capability \& skill, occupational health and security, technical capacity, managerial capacity, past performance, and past experience. As seen from the above representative literature, quotation, technical strength and resource strength are the three main factors which are widely accepted and adopted; we thus include these factors as main evaluative attributes in this paper. Another finding from the above reviewed literature is that nearly all of them emphasized the inclusion of indicators to examine credibility of contractors in their managing external and internal challenges [72, 73], such as financial capability [24], safety performance [24], and reputation [10, 25, 26]; therefore, we introduce the 'Credibility' as another main attribute in this paper.

Indeed, cooperation attributes must be taken into consideration because cooperation among selected contractor influences the project success [74]. Actually, some of earlier pioneering studies also noticed and took consideration of cooperation attribute in their parameter systems, such as 'client/contractor relationship' in references [10, 24]. Recently, increasing attentions have been paid to investigate appropriate evaluative cooperation attributes for contractor selection. Representatively, based on the conceptual model of partnering and alliancing in construction project management, Liang et al. [8] elaborated a set of cooperation attributes for selecting joint venture contractors in largescale infrastructure projects, including compatible culture, contract, communication, collaboration, cooperation ability and cooperation satisfaction. To provide a deeper insights 
on factors that affect contractors' cooperation under international construction joint ventures in construction projects, Hwang et al. [27] conducted a survey and reported 'sharing of project risks' as the top attractive factor and 'differences in culture and working style' as the top negative factor among others. Furthermore, aiming at finding risks that most affect contractors' cooperation within project networks, Hwang and Han [29] conducted a survey from the viewpoint of contractors and sub-contractors in Singapore construction environment; they identified ten top critical network risks, such as 'different cultural norms', 'inaccurate information delivery,' 'occurrence of dispute,' and 'lack of risk management knowledge.' Obviously, Hwang, et al. [27] and Hwang and Han [29] contributed a feasible way to deduce more reasonable evaluative attributes on contractor's cooperation capability; however, to our best knowledge, there is still a lack of thorough investigation on a consensus set of evaluative attributes on contractor's cooperation capability for referencing. Therefore, in the light of Liang et al. [8], Hwang, et al. [27], and Hwang and Han [29], we here take 'Cooperation management capability' as one of the main evaluative attributes to assess contractors.

To reduce the significant environmental footprint [6], internal and external factors (such as government regulations and managerial concerns) are driving the entire construction industry to enforce green practices all over the world [6]. Green practices can be considered as an outcome of strategic processes through cooperation within the nexus of contractors [75]. Therefore, it is intrinsically crucial to take into account evaluative attributes on green practices in contractor selection, especially for those nearly ever-lasting mega infrastructure construction projects. Since environmental regulations of different scales (i.e., domestic, governmental, and international [76]) have increasingly become compulsory in various industrial markets, contractors should have the capability of manage and keep track of the compliance [35]. And the widely accepted way [77] to attain the compliance capability by building environment management systems $[32,34,78]$, whose most-cited components [77] comprise of ISO-14001 certification [32, 34, 36, 37], eco-labeling [31, 32,37 , environment policies [33], environment planning [33], and environmental management information system (continuous monitoring and regulatory compliance) [31, 32]. Due to the reason that environmental impacts occur at every stage of the construction cycle, Rwelamila et al. [79] strongly suggested to contractors should implement green design \& procurement to improve their green practices. Construction life-cycle analysis is critical in both green design for environment [33] and pollution reduction through green procurement $[31,32,34]$. Measurements of green design for environment include tracking all material and reverse flow of a project from the retrieval of raw materials out of the environment to the disposal of the product back into the environment, generally including recycle [31], reuse $[32,33]$, remanufacture $[32,33]$, disassembly and disposal [33]. In the green procurement aspect, Tan, et al. [68] suggested to reduce environmental footprints throughout the whole construction supply chain by addressing issues such as waste reduction [32, 34], environment material substitution [31, 33], hazardous material minimization [35], and clean technology availability [33]. Furthermore, from the stance of strategic management, Fergusson and Langford [80] pointed out environmental performance of green practices positively contributes to comprehensive competitive advantages of contractors. Environmental performance has thus been recognized as one of the indispensible evaluative attributes to contractor selection [34, 78]. Sharma and Vredenburg [81] referred to environmental performance as the environmental effects that corporation's activities have on the natural milieu. To make environmental performance more measurable, many efforts have been paid to establish effective assessing approaches, such as the approach to examination on waste flows and control on construction sites $[3,82]$. As for indicators of environmental performance, according to the thorough literature review by Govindan, et al. [77], the commonly adopted ones include [32-34, 36]: solid waste, chemical waste, air emission, waste water disposal and energy. In sum, we here include 'Design \& procurement,' 'Compliance with green legislation,' and 'Environmental performance' as three other main evaluative attributes to comprehensively assess green practices of alternative contractors.

For more clarity, based on the above literature review, we listed all eight main evaluative attributes and present their definitions/principles. And the optional subdimensions of these main attributes have been also listed in Table 1 for various comprehensive considerations according to specific nature in practical problems.

$\left(A_{1}\right)$ Quotation: Project value of reviewed. The lowest tender price tends to attract a client's interest as superior to other criteria.

$\left(A_{2}\right)$ Credibility: Comprehensive evaluation on trustworthiness of contractors in developmental dynamics and risks from both internal and external environments, focusing on financial soundness, health and safety, past performance, work experience, etc.

$\left(\mathrm{A}_{3}\right)$ Technical strength: Comprehensive evaluation on contractors' capability of technology and innovation that tackling forthcoming complicate tasks, generally based on quality rank, technical ability, experience and knowledge of operation team, creativity \& innovation, etc.

$\left(\mathrm{A}_{4}\right)$ Resource strength: Comprehensive evaluation on contractors' competitive strength on productive resources that indispensable for construction needs, generally based on technical human resource, current workload, construction machinery \& equipment, fixed assets \& liquidity, etc.

$\left(\mathrm{A}_{5}\right)$ Cooperation management capacity: Comprehensive evaluation on contractors' cooperation practices with participators in projects, focusing on client/contractor relationship, organizational structure and operations, compatible culture, communication and information delivery, knowledge sharing of risk management, among others.

$\left(\mathrm{A}_{6}\right)$ Design \& procurement: Comprehensive evaluation on contractors' practices that improve project's whole life value by using green design, environmental friendly materials and green production processes, which promote best practices of green construction procurement throughout the supply chain. 


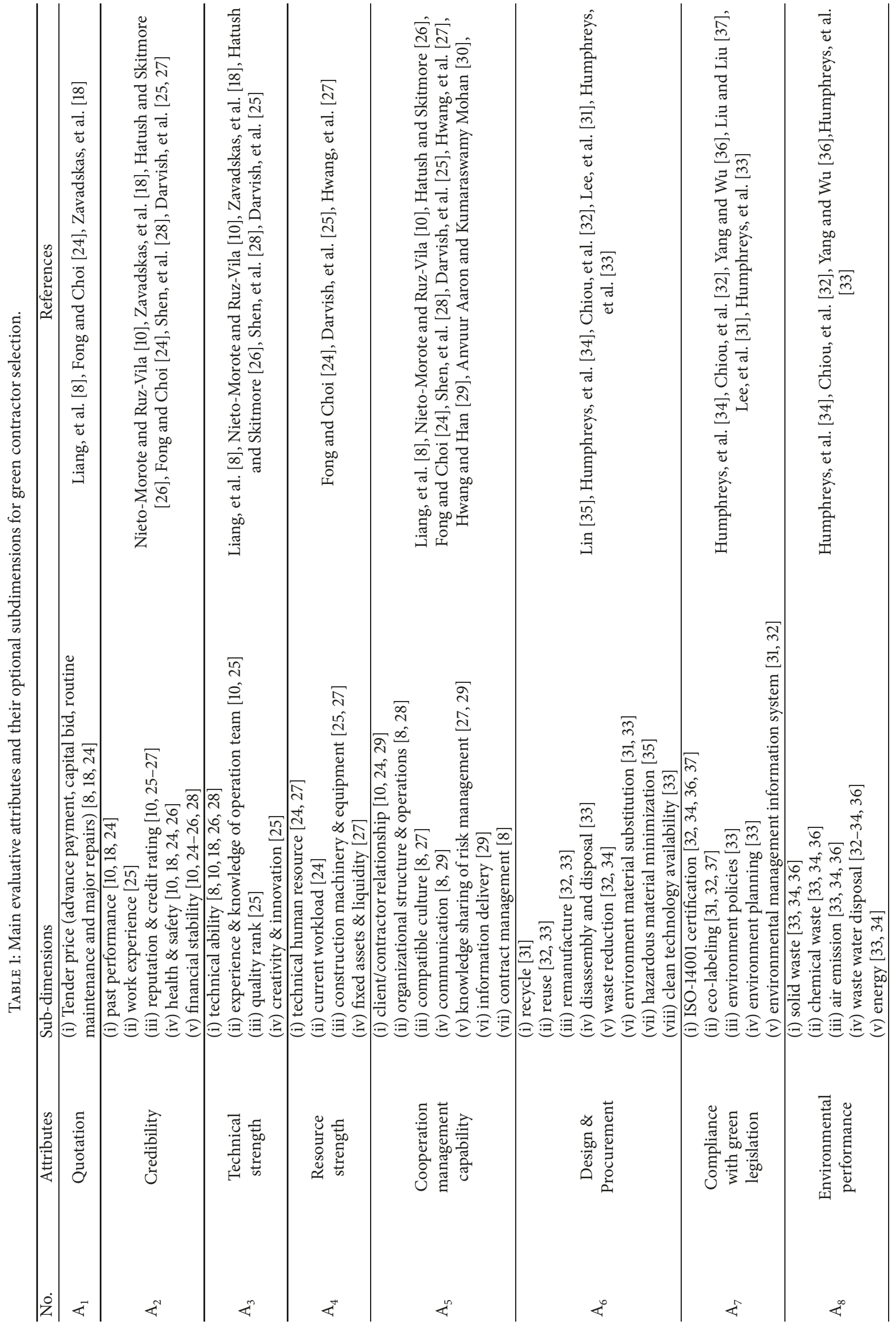


$\left(A_{7}\right)$ Compliance with green legislation: Comprehensive evaluation on the extent to which contractors' practices satisfy different governmental green or sustainability legislations, according to the aspects of ISO-14001 certification, eco-labeling, environment policies, environment planning, environmental management information system, etc.

$\left(A_{8}\right)$ Environmental performance: Comprehensive evaluation on environmental effects that the corporation's activities have on the natural milieu. Environmental performance can commonly be measured through operative performance indicators (i.e. energy/resource utilization, emission reduction, and waste disposal).

2.2. Problem Definition. The contractor selection as well as many other multicriteria decisions impacting the overall project should be made during the front end planning stage of a project, the point at which a group of designated decisionmakers have the power to accept or reject a contractor for a specific project or its work packages $[5,9]$. When using MADM mechanism to cope with complicate contractor selection problems [5], normally few nominated contractors will be ready for the decision-makers to vote on. Due to complexity of the problems and limitedness of knowledge, decision-makers usually feel confident in expressing their opinions by use of interval numbers [83] or uncertain linguistic terms [52]. Although widely-accepted majority rule will quickly help the group of decision-makers arrive at a decision on an uncertain linguistic term (e.g., $\left[s_{4}, s_{5}\right]$ ), to which obviously there exists decision hesitancy because different opinions exist. Therefore, in this paper, we define the intervalvalued dual hesitant fuzzy uncertain unbalanced linguistic set (IVDHF_UUBLS) to help the decision-making panel elicit their assessments more objectively and completely.

From another point of view, various attitudinal characters (degree of orness) commonly exist because individual expert holds specific backgrounds, and decision process that involves the attitudinal character of group decisionmakers must coordinate those various attitudinal characters into one complex attitudinal character [61]. Therefore, we adopt the concept of order-inducing vector [62] to reflect group complex attitudinal character and develop a TOPSISbased method to rationally determine the order-inducing vector. Besides, as Tan and Chen [84] pointed out, for real-world sophisticated MADM problems, the independency axiom [85] cannot generally satisfied. For example, upgrading in green performance will raise the quotation and intrinsically result in requirements for high-standard collaboration between contractors and general constructor. In viewing of this common phenomenon, we take attributes' interdependency as a third indispensible characteristic in tackling complexity in green contractor selection in mega infrastructure projects. In sum, we take three characteristics of complexity to model the practical problems of complicate green contractor selection, i.e., (i) compound structure of hesitant fuzzy linguistic assessments, (ii) group attitudinal characters, and (iii) attributes' interdependency. To produce greater clarity, Figure 1 demonstrates the conceptual MADM model for green contractor selection.
Now, we can give the symbolized description of the targeted complex green contractor problems. Given a mega infrastructure project, there are a set of alternative green contractors, i.e., $X=\left\{x_{1}, x_{2}, \ldots, x_{n}\right\}$, for its subprojects. Let $A=\left\{A_{1}, A_{2}, \ldots, A_{m}\right\}$ be the evaluative attributes according to which decision-makers consider each green contractor. Due to high complexity in the sophisticated problem scenarios, there exists interdependency relations among the evaluative attribute.. A panel of decision-makers $E=\left\{E_{1}, E_{2}, \ldots, E_{t}\right\}$ have been organized to give their assessments to each alternative contractor $x_{i}(i=1, \ldots, n)$ under every attribute $A_{j}(j=1, . ., m)$. In order to reflect the complicate group assessments of all alternative contactors, the hybrid expression tool of IVDHF_UUBLS that will be detailed in Section 3 is adopted to depict the assessments more effectively and comprehensively. As a result, a specific decision matrix $R=\left(r_{i j}\right)_{m \times n}$ whose elements are in the form of IVDHF_UUBLS is obtained. According to Merigó and Casanovas [61], suppose that an order-inducing vector $\varepsilon$ for denoting group attitudinal characters has been reasonably obtained. Then, effective MADM approaches must be developed to determine the most appropriate green contractor(s).

\section{Interval-Valued Dual Hesitant Fuzzy Uncertain Unbalanced Linguistic Set}

As demonstrated in Figure 1, after the panel of decisionmakers votes on an alternative contractor under certain attribute by use of specific uncertain unbalanced linguistic term set, the uncertain linguistic term $\left[s_{4}, s_{5}\right]$ stands out because of the majority rule while different opinions should also be included and considered in MADM processes. To that end, based on interval-valued dual hesitant fuzzy set (IVDHFS) [47] and the unbalanced linguistic term set (ULTS) [59], we here first introduce an interval-valued dual hesitant fuzzy uncertain unbalanced linguistic set (IVDHF_UUBLS), which incorporate different opinions of decision-makers as membership degrees or nonmembership degrees to the majority-voted $\left[s_{4}, s_{5}\right]$. Then we develop operational rules as well as distance measure for the IVDHF_UUBLS. Regarding definitions of the IVDHFS and ULTS, one can refer to Appendix A.

\subsection{Definition of IVDHF_UUBLS}

Definition 1. Let $X$ be a fixed set and $S$ be a finite and continuous unbalanced linguistic label set. Then an IVDHF_UUBLS $S D$ on $X$ is defined as

$$
S D=\left\{\left\langle x, \widetilde{s}_{\vartheta(x)}, \widetilde{h}(x), \widetilde{g}(x)\right\rangle \mid x \in X\right\},
$$

where $\widetilde{s}_{\theta(x)}=\left[s_{\alpha}, s_{\beta}\right]$ represents judgment to object $x$, $s_{\alpha}$ and $s_{\beta}$ are two unbalanced linguistic variables from predefined unbalanced linguistic label set $S$ which represents decision-makers' judgments to an evaluated object $X . \tilde{h}(x)=\bigcup\{\widetilde{\mu}\}=\bigcup_{\left[\mu^{L}, \mu^{U}\right] \in \tilde{h}(x)}\left\{\left[\mu^{L}, \mu^{U}\right]\right\}$ and $\widetilde{g}(x)=$ $\bigcup\{\tilde{\nu}\}=\bigcup_{\left[\nu^{L}, \nu^{U}\right] \in \tilde{g}(x)}\left\{\left[\nu^{L}, \nu^{U}\right]\right\}$ are two sets of closed intervals 


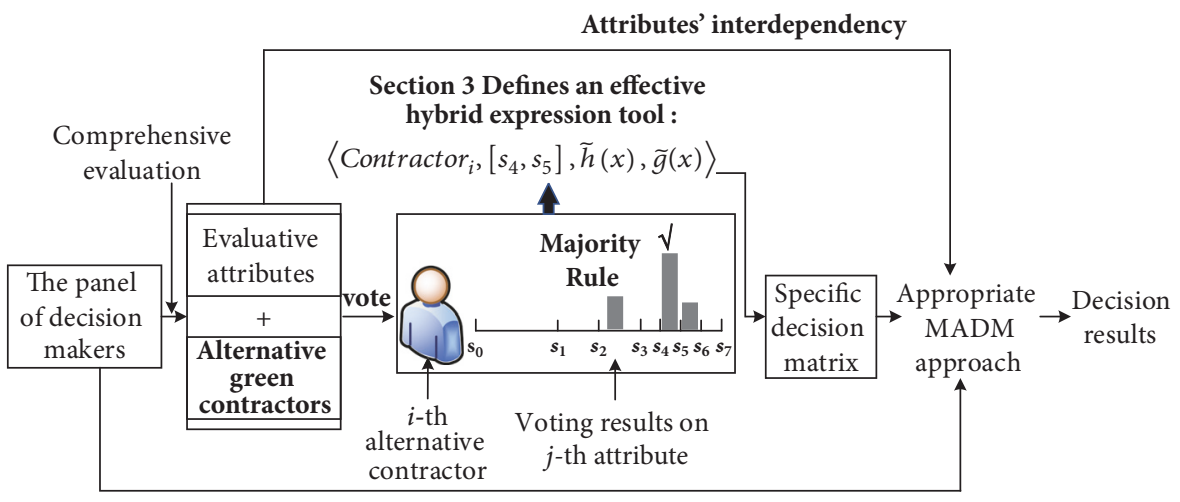

Group attitudinal characters (i.e., complex degrees of orness)

FIGURE 1: Conceptual MADM model for tackling complexity in green contractor selection.

in $[0,1] . \widetilde{h}(x)$ denotes possible membership degrees that $x$ belongs to $\widetilde{s}_{\theta(x)}$, and $\widetilde{g}(x)$ represents possible nonmembership degrees of $x$ to $\widetilde{s}_{\theta(x)} . \widetilde{h}(x)$ and $\widetilde{g}(x)$ hold conditions: $\widetilde{\mu}, \widetilde{v} \in$ $[0,1]$ and $0 \leq\left(\mu^{U}\right)^{+}+\left(\nu^{U}\right)^{+} \leq 1$, where $\left(\mu^{U}\right)^{+} \epsilon$ $\widetilde{h}^{+}(x)=\bigcup_{\left[\mu^{L}, \mu^{U}\right] \in \widetilde{h}(x)} \max \left\{\mu^{U}\right\}$ and $\left(\nu^{U}\right)^{+} \in \tilde{g}^{+}(x)=$ $\bigcup_{\left[\nu^{L}, \nu^{U}\right] \in \tilde{g}(x)} \max \left\{\nu^{U}\right\}$ for all $x \in X$. When $X=\left\{x_{1}, x_{2}, \ldots, x_{n}\right\}$ has only one element, $S D$ reduces to $\left(\widetilde{\mathrm{s}_{9}}, \widetilde{h}, \widetilde{g}\right)$, which is called an interval-valued dual hesitant fuzzy uncertain unbalanced linguistic (IVDHF_UUBL) number (IVDHF_UUBLN).
3.2. Operational Rules for IVDHF_UUBLS. On the strength of operational rules for uncertain linguistic set [52], unbalanced linguistic set [59] and interval-valued dual hesitant fuzzy set [47], we get the following operations for IVDHF_UUBLS.

Definition 2. Let $s d=\left(\widetilde{s}_{\vartheta}, \widetilde{h}, \widetilde{g}\right)=\left(\left[s_{\alpha}, s_{\beta}\right], \widetilde{h}, \widetilde{g}\right), s d_{1}=$ $\left(\widetilde{s}_{\vartheta_{1}}, \widetilde{h}_{1}, \widetilde{g}_{1}\right)=\left(\left[s_{\alpha_{1}}, s_{\beta_{1}}\right], \widetilde{h}_{1}, \widetilde{g}_{1}\right)$ and $s d_{2}=\left(\widetilde{s}_{\vartheta_{2}}, \widetilde{h}_{2}, \widetilde{g}_{2}\right)=$ $\left(\left[s_{\alpha_{2}}, s_{\beta_{2}}\right], \widetilde{h}_{2}, \widetilde{g}_{2}\right)$ be any three IVDHF_UUBLNs, $\lambda \in[0,1]$, operations on these IVDHF_UUBLNs are defined as:

(1) $\quad \lambda s d=\bigcup_{\left(\widetilde{s}_{g}, \tilde{h}, \tilde{g}\right) \in s d}\left(\left[s_{\lambda \Delta_{t_{0}}^{-1}\left(T F_{t_{0}}^{t_{k_{1}}}\left(\psi\left(s_{\alpha}\right)\right)\right)}, s_{\lambda \Delta_{t_{0}}^{-1}\left(T t_{t_{0}}^{t_{k 2}}\left(\psi\left(s_{\beta}\right)\right)\right)}\right]\right.$,

$\left.\bigcup_{\left[\mu^{L}, \mu^{U}\right] \in \widetilde{h},\left[\nu^{L}, \nu^{U}\right] \in \tilde{g}}\left\{\left\{\left[1-\left(1-\mu^{L}\right)^{\lambda}, 1-\left(1-\mu^{U}\right)^{\lambda}\right]\right\},\left\{\left[\left(\nu^{L}\right)^{\lambda},\left(\nu^{U}\right)^{\lambda}\right]\right\}\right\}\right)$

(2) $\quad s d^{\lambda}=\bigcup_{\left(\tilde{s_{g}}, \widetilde{h}, \tilde{g}\right) \in s d}\left(\left[s_{\left(\Delta_{t_{0}}^{-1}\left(T F_{t_{0}}^{t_{k_{1}}}\left(\psi\left(s_{\alpha}\right)\right)\right)\right)^{\lambda}}, s_{\left(\Delta_{t_{0}}^{-1}\left(T F_{t_{0}}^{t_{2}}\left(\psi\left(s_{\beta}\right)\right)\right)\right)^{\lambda}}\right]\right)$

$\left.\bigcup_{\left[\mu^{L}, \mu^{U}\right] \in \widetilde{h},\left[\nu^{L}, \nu^{U}\right] \in \tilde{g}}\left\{\left\{\left[\left(\mu^{L}\right)^{\lambda},\left(\mu^{U}\right)^{\lambda}\right]\right\},\left\{\left[1-\left(1-\nu^{L}\right)^{\lambda}, 1-\left(1-\nu^{U}\right)^{\lambda}\right]\right\}\right\}\right) ;$

(3) $s d_{1} \oplus s d_{2}=\bigcup_{\left(\widetilde{s}_{s_{1}}, \widetilde{h}_{1}, \widetilde{g}_{1}\right) \in s d_{1},\left(\widetilde{s}_{s_{2}}, \widetilde{h}_{2}, \widetilde{g}_{2}\right) \in s d_{2}}\left(\left[s_{\Delta_{t_{0}}^{-1}\left(T_{t_{0}}^{t_{1}}\left(\psi\left(s_{\alpha_{1}}\right)\right)\right)+\Delta_{t_{0}}^{-1}\left(T F_{t_{0}}^{t_{1}}\left(\psi\left(s_{\alpha_{2}}\right)\right)\right)}, s_{\Delta_{t_{0}}^{-1}\left(T F_{t_{0}}^{t_{2}}\left(\psi\left(s_{\beta_{1}}\right)\right)\right)+\Delta_{t_{0}}^{-1}\left(T F_{t_{0}}^{t_{j_{2}}}\left(\psi\left(s_{\beta_{2}}\right)\right)\right)}\right]\right.$,

$\left.\bigcup_{\left[\mu_{1}^{L}, \mu_{1}^{U}\right] \in \widetilde{h}_{1},\left[\mu_{2}^{L}, \mu_{2}^{U}\right] \in \widetilde{h}_{2},\left[\nu_{1}^{L}, \nu_{1}^{U}\right] \in \widetilde{g}_{1},\left[\nu_{2}^{L}, \nu_{2}^{U}\right] \in \widetilde{g}_{2}}\left\{\left\{\left[\mu_{1}^{L}+\mu_{2}^{L}-\mu_{1}^{L} \mu_{2}^{L}, \mu_{1}^{U}+\mu_{2}^{U}-\mu_{1}^{U} \mu_{2}^{U}\right]\right\},\left\{\left[\nu_{1}^{L} v_{2}^{L}, v_{1}^{U} v_{2}^{U}\right]\right\}\right\}\right) ;$

(4) $s d_{1} \otimes s d_{2}=\bigcup_{\left(\widetilde{s}_{1_{1}}, \widetilde{h}_{1}, \widetilde{g}_{1}\right) \in s d_{1},\left(\widetilde{s}_{s_{2}}, \widetilde{h}_{2}, \widetilde{g}_{2}\right) \in s d_{2}}\left(\left[s_{\Delta_{t_{0}}^{-1}\left(T F_{t_{0}}^{t_{1}}\left(\psi\left(s_{\alpha_{1}}\right)\right)\right) \times \Delta_{t_{0}}^{-1}\left(T F_{t_{0}}^{t_{1}}\left(\psi\left(s_{\alpha_{2}}\right)\right)\right)}, s_{\Delta_{t_{0}}^{-1}\left(T F_{t_{0}}^{t_{t_{2}}}\left(\psi\left(s_{\beta_{1}}\right)\right)\right) \times \Delta_{t_{0}}^{-1}\left(T F_{t_{0}}^{j_{2}}\left(\psi\left(s_{\beta_{2}}\right)\right)\right)}\right]\right.$,

$\left.\bigcup_{\left[\mu_{1}^{L}, \mu_{1}^{U}\right] \in \widetilde{h}_{1},\left[\mu_{2}^{L}, \mu_{2}^{U}\right] \in \widetilde{h}_{2},\left[v_{1}^{L}, \nu_{1}^{U}\right] \in \tilde{g}_{1},\left[v_{2}^{L}, \nu_{2}^{U}\right] \in \widetilde{g}_{2}}\left\{\left\{\left[\mu_{1}^{L} \mu_{2}^{L}, \mu_{1}^{U} \mu_{2}^{U}\right]\right\},\left\{\left[\nu_{1}^{L}+v_{2}^{L}-v_{1}^{L} v_{2}^{L}, v_{1}^{U}+v_{2}^{U}-v_{1}^{U} v_{2}^{U}\right]\right\}\right\}\right)$. 
In above, details about the transformation functions of $T F$ and $\Delta$, linguistic hierarchies $(L H)$ as well as the transformation procedures for unbalanced linguistic term sets are shown in Appendix A.

Theorem 3. Letting $s d=\left(\widetilde{s}_{\vartheta}, \widetilde{h}, \widetilde{g}\right), s d_{1}=\left(\widetilde{s}_{\vartheta_{1}}, \widetilde{h}_{1}, \widetilde{g}_{1}\right)$, and $s d_{2}=\left(\widetilde{s}_{\vartheta_{2}}, \tilde{h}_{2}, \widetilde{g}_{2}\right)$ be any three IVDHF_UUBLNs, $\lambda, \lambda_{1}, \lambda_{2} \in$ $[0,1]$, then following properties are true:

(1) $s d_{1} \oplus s d_{2}=s d_{2} \oplus s d_{1} ;(2) s d_{1} \otimes s d_{2}=s d_{2} \otimes s d_{1}$; (3) $\lambda\left(s d_{1} \oplus s d_{2}\right)=\lambda s d_{1} \oplus \lambda s d_{2}$;

(4) $s d_{1}^{\lambda} \otimes s d_{2}^{\lambda}=\left(s d_{1} \otimes s d_{2}\right)^{\lambda}$; (5) $\lambda_{1} s d \oplus \lambda_{2} s d=\left(\lambda_{1}+\right.$ $\left.\lambda_{2}\right) s d ;(6) s d^{\lambda_{1}} \otimes s d^{\lambda_{2}}=s d^{\lambda_{1}+\lambda_{2}}$.

Proof. Omitted

In Definition 2 and Theorem 3, $t_{k_{1}}, t_{k_{2}}, t_{i_{1}}, t_{i_{2}}, t_{j_{1}}$, and $t_{j_{2}}$ are corresponding levels of $s_{\alpha}, s_{\beta}, s_{\alpha_{1}}, s_{\beta_{1}}, s_{\alpha_{2}}$, and $s_{\alpha_{2}}$ in $L H$, respectively. $t_{0}$ is the maximum level of $s_{\alpha}, s_{\beta}, s_{\alpha_{1}}$, $s_{\beta_{1}}, s_{\alpha_{2}}$, and $s_{\alpha_{2}}$ in $L H$. Furthermore, to compare any two IVDHF_UUBLNs, we also have following definitions.

Definition 4. Let $s d=\left(\widetilde{s_{9}}, \tilde{h}, \widetilde{g}\right)$ be an IVDHF_UUBLN, $\widetilde{s}_{\vartheta}=\left[s_{\alpha}, s_{\beta}\right]$; then score function $S(s d)$ and accuracy function $P(s d)$ can be represented by

$$
\begin{aligned}
& S(s d)=\frac{\Delta_{t_{0}}^{-1}\left(T F_{t_{0}}^{t_{k_{1}}}\left(\psi\left(s_{\alpha}\right)\right)\right)+\Delta_{t_{0}}^{-1}\left(T F_{t_{0}}^{t_{k_{2}}}\left(\psi\left(s_{\beta}\right)\right)\right)}{2}, \\
& \times \frac{1}{2}\left(\frac{1}{l(\widetilde{h})} \sum_{\left[\mu^{L}, \mu^{U}\right] \in \widetilde{h}} \mu^{L}-\frac{1}{l(\widetilde{g})} \sum_{\left[\nu^{L}, \nu^{U}\right] \in \widetilde{g}} \nu^{L}\right. \\
& \left.+\frac{1}{l(\widetilde{h})} \sum_{\left[\mu^{L}, \mu^{U}\right] \in \tilde{h}} \mu^{U}-\frac{1}{l(\widetilde{g})} \sum_{\left[\nu^{L}, \nu^{U}\right] \in \widetilde{g}} \nu^{U}\right)
\end{aligned}
$$

$P(s d)$

$$
\begin{aligned}
& =\frac{\Delta_{t_{0}}^{-1}\left(T F_{t_{0}}^{t_{k_{1}}}\left(\psi\left(s_{\alpha}\right)\right)\right)+\Delta_{t_{0}}^{-1}\left(T F_{t_{0}}^{t_{k_{2}}}\left(\psi\left(s_{\beta}\right)\right)\right)}{2}, \\
& \times \frac{1}{2}\left(\frac{1}{l(\widetilde{h})} \sum_{\left[\mu^{L}, \mu^{U}\right] \in \widetilde{h}} \mu^{L}+\frac{1}{l(\widetilde{g})} \sum_{\left[\nu^{L}, \nu^{U}\right] \in \widetilde{g}} v^{L}\right. \\
& \left.+\frac{1}{l(\widetilde{h})} \sum_{\left[\mu^{L}, \mu^{U}\right] \in \tilde{h}} \mu^{U}+\frac{1}{l(\widetilde{g})} \sum_{\left[\nu^{L}, \nu^{U}\right] \in \tilde{g}} \nu^{U}\right)
\end{aligned}
$$

where $l(\tilde{h})$ and $l(\widetilde{g})$ are numbers of values in $\tilde{h}$ and $\widetilde{g}$, respectively, $t_{k_{1}}$ and $t_{k_{2}}$ are the corresponding levels of $s_{\alpha}$ and $s_{\beta}$ in $L H$, and $t_{0}$ is the maximum level of $t_{k}$ in $L H$.

Definition 5. Given any IVDHF_UUBLNs $s d_{1}=\left(\widetilde{s}_{\vartheta_{1}}, \widetilde{h}_{1}, \widetilde{g}_{1}\right)$, $s d_{2}=\left(\widetilde{s}_{\vartheta_{2}}, \widetilde{h}_{2}, \widetilde{g}_{2}\right)$, then

(1) If $S\left(s d_{1}\right)<S\left(s d_{2}\right)$, then $s d_{1}<s d_{2}$.

(2) If $S\left(s d_{1}\right)=S\left(s d_{2}\right)$, then (a) If $P\left(s d_{1}\right)=P\left(s d_{2}\right)$, then $s d_{1}=s d_{2}$;

(b) If $P\left(s d_{1}\right)<P\left(s d_{2}\right)$, then $s d_{1}<s d_{2}$.

3.3. Distance Measure for IVDHF_UUBLS. When $l(\widetilde{h})$ or $l(\widetilde{g})$ of two IVDHF_UUBLNs are unequal, the complementing method [86] is normally adopted to design distance measures. Note that artificially adding values to shorter ones in the complementing method will cause information distortion. To avoid this limitation, we provide the following distance measure.

Definition 6. Let two IVDHF_UUBLNs $s d_{1}=\left(\widetilde{s}_{\vartheta_{1}}, \widetilde{h}_{1}, \widetilde{g}_{1}\right)$ and $s d_{2}=\left(\widetilde{s}_{\vartheta_{2}}, \widetilde{h}_{2}, \widetilde{g}_{2}\right)$, where $\widetilde{s}_{\vartheta_{1}}=\left[s_{\alpha_{1}}, s_{\beta_{1}}\right], \widetilde{s}_{\vartheta_{2}}=\left[s_{\alpha_{2}}, s_{\beta_{2}}\right]$. $l_{\widetilde{h}_{1}}, l_{\widetilde{h}_{2}}, l_{\widetilde{g}_{1}}$ and $l_{\widetilde{g}_{2}}$ are lengths of $\widetilde{h}_{1}, \widetilde{h}_{2}, \widetilde{g}_{1}$, and $\widetilde{g}_{2}$, respectively, denoting number of elements in $\tilde{h}_{1}, \tilde{h}_{2}, \widetilde{g}_{1}$, and $\widetilde{g}_{2}$. Suppose $I_{1}=\left(1 /\left(n\left(t_{i_{1}}\right)-1\right)\right) \Delta_{t_{0}}^{-1}\left(T F_{t_{0}}^{t_{i_{1}}}\left(\psi\left(s_{\alpha_{1}}\right)\right)\right), I_{2}=\left(1 /\left(n\left(t_{i_{2}}\right)-\right.\right.$ $1)) \Delta_{t_{0}}^{-1}\left(T F_{t_{0}}^{t_{i_{2}}}\left(\psi\left(s_{\beta_{1}}\right)\right)\right), I_{3}=\left(1 /\left(n\left(t_{j_{1}}\right)-1\right)\right) \Delta_{t_{0}}^{-1}\left(T F_{t_{0}}^{t_{j_{1}}}\left(\psi\left(s_{\alpha_{2}}\right)\right)\right)$, $I_{4}=\left(1 /\left(n\left(t_{j_{2}}\right)-1\right)\right) \Delta_{t_{0}}^{-1}\left(T F_{t_{0}}^{t_{j_{2}}}\left(\psi\left(s_{\beta_{2}}\right)\right)\right)$, where $t_{i_{1}}, t_{i_{2}}, t_{j_{1}}$, and $t_{j_{2}}$ are the corresponding levels of unbalanced linguistic terms $s_{\alpha_{1}}, s_{\beta_{1}}, s_{\alpha_{2}}$, and $s_{\beta_{2}}$ in the linguistic hierarchy $L H$ and $t_{0}$ is the maximum level of $s_{\alpha_{1}}, s_{\beta_{1}}, s_{\alpha_{2}}$, and $s_{\beta_{2}}$ in $L H$. Then by use of normalized Euclidean distance, we define a distance measure $d$ for IVDHF_UUBLNs as follows.

Situation 7. When $l_{\widetilde{h}_{1}}=l_{\widetilde{h}_{2}}=l_{1}$ and $l_{\widetilde{g}_{1}}=l_{\widetilde{g}_{2}}=l_{2}$, then

$$
\begin{aligned}
& d\left(s d_{1}, s d_{2}\right)=\left(\frac { 1 } { 2 } \left(\frac{1}{l_{1}}\right.\right. \\
& \cdot \sum_{k=1}^{l_{1}}\left(\left|I_{1} \mu_{\tilde{h}_{1}}^{L_{j}}-I_{3} \mu_{\tilde{h}_{2}}^{L_{k}}\right|^{2}+\left|I_{2} \mu_{\tilde{h}_{1}}^{U_{j}}-I_{4} \mu_{\tilde{h}_{2}}^{U_{k}}\right|^{2}\right)+\frac{1}{l_{2}} \\
& \left.\left.\quad \cdot \sum_{k=1}^{l_{2}}\left(\left|I_{1} \nu_{\widetilde{g}_{1}}^{L_{j}}-I_{3} \nu_{\tilde{g}_{2}}^{L_{k}}\right|^{2}+\left|I_{2} \nu_{\widetilde{g}_{1}}^{U_{j}}-I_{4} \nu_{\tilde{g}_{2}}^{U_{k}}\right|^{2}\right)\right)\right)^{1 / 2} .
\end{aligned}
$$

Situation 8. When $l_{\widetilde{h}_{1}} \neq l_{\widetilde{h}_{2}}$ or $l_{\widetilde{g}_{1}} \neq l_{\widetilde{g}_{2}}$, then

$$
\begin{aligned}
& d\left(s d_{1}, s d_{2}\right)=\left(\frac { 1 } { 2 } \left(\frac{1}{l_{\widetilde{h}_{1}}{\widetilde{h_{2}}}_{2}}\right.\right. \\
& \quad \cdot \sum_{j=1}^{l_{\tilde{h}_{1}}} \sum_{k=1}^{l_{\tilde{h}_{2}}}\left(\left|I_{1} \mu_{\widetilde{h}_{1}}^{L_{j}}-I_{3} \mu_{\widetilde{h}_{2}}^{L_{k}}\right|^{2}+\left|I_{2} \mu_{\widetilde{h}_{1}}^{U_{j}}-I_{4} \mu_{\widetilde{h}_{2}}^{U_{k}}\right|^{2}\right) \\
& +\frac{1}{l_{\widetilde{g}_{1}} l_{\widetilde{g}_{2}}} \\
& \left.\quad \cdot \sum_{j=1}^{l_{\widetilde{g}_{1}}} \sum_{k=1}^{l_{\widetilde{g}_{2}}}\left(\left|I_{1} \nu_{\widetilde{g}_{1}}^{L_{j}}-I_{3} \nu_{\widetilde{g}_{2}}^{L_{k}}\right|^{2}+\left|I_{2} \nu_{\widetilde{g}_{1}}^{U_{j}}-I_{4} \nu_{\widetilde{g}_{2}}^{U_{k}}\right|^{2}\right)\right)^{1 / 2} .
\end{aligned}
$$

Theorem 9. The distance measure $d$ defined in Definition 6 satisfies following properties:

(1) $0 \leq d\left(s d_{1}, s d_{2}\right) \leq 1$; 
(2) $d\left(s d_{1}, s d_{2}\right)=0$ if and only if $s d_{1}$ and $s d_{2}$ are perfectly consistent;

(3) $d\left(s d_{1}, s d_{2}\right)=d\left(s d_{2}, s d_{1}\right)$.

\section{Generalized Aggregation Operators for IVDHF_UUBLS}

4.1. Definitions of Operators. Based on the generalized operators firstly introduced by Yager [87], we here develop some fundamental generalized aggregation operators for the newly defined IVDHF_UUBLS.

Definition 10. Given a collection of IVDHF_UUBLNs $s d_{j}(j=$ $1, \ldots, n)$, their weighting vector $\omega=\left(\omega_{1}, \omega_{2}, \ldots, \omega_{n}\right)^{T}, \omega_{j} \in$ $[0,1], \sum_{j=1}^{n} \omega_{j}=1$. $\lambda$ be a parameter, $\lambda \in(0,+\infty)$.

(1) Generalized IVDHFUUBL Weighted Average (GIVDHFUUBLWA) Operator

$$
\begin{aligned}
& \text { GIVDHFUUBLWA } \omega_{\omega, \lambda}\left(s d_{1}, s d_{2}, \ldots, s d_{n}\right)=\left(\bigoplus_{j=1}^{n}\left(\omega_{j} s d_{j}^{\lambda}\right)\right)^{1 / \lambda} \\
& =\bigcup_{\left(\tilde{s}_{s_{j}}, \tilde{h}_{j}, \tilde{g}_{j}\right) \in s d_{j}}\left(\left[s_{\left(\sum_{j=1}^{n} \omega_{j}\left(\Delta_{t_{0}}^{-1}\left(T t_{t_{0}}^{t_{j_{1}}}\left(\psi\left(s_{\alpha_{j}}\right)\right)\right)^{\lambda}\right)^{1 / \lambda}, s_{\left(\sum_{j=1}^{n} \omega_{j}\left(\Delta_{t_{0}}^{-1}\left(T F_{t_{0}}^{t_{2}}\left(\psi\left(s_{\beta_{j}}\right)\right)\right)\right)^{\lambda}\right)^{1 / \lambda}}\right],}\right.\right. \\
& \bigcup_{\left[\mu_{j}^{L}, \mu_{j}^{U}\right] \in \widetilde{h}_{j},\left[\nu_{j}^{L}, \nu_{j}^{U}\right] \in \tilde{g}_{j}}\left\{\left\{\left[\left(1-\prod_{j=1}^{n}\left(1-\left(\mu_{j}^{L}\right)^{\lambda}\right)^{\omega_{j}}\right)^{1 / \lambda},\left(1-\prod_{j=1}^{n}\left(1-\left(\mu_{j}^{U}\right)^{\lambda}\right)^{\omega_{j}}\right)^{1 / \lambda}\right]\right\},\right. \\
& \left.\left.\left\{\left[1-\left(1-\prod_{j=1}^{n}\left(1-\left(1-v_{j}^{L}\right)^{\lambda}\right)^{\omega_{j}}\right)^{1 / \lambda}, 1-\left(1-\prod_{j=1}^{n}\left(1-\left(1-v_{j}^{U}\right)^{\lambda}\right)^{\omega_{j}}\right)^{1 / \lambda}\right]\right\}\right\}\right),
\end{aligned}
$$

(2) Generalized IVDHFUUBL Weighted Geometric (GIVDHFUUBLWG) Operator

$$
\begin{aligned}
& \text { GIVDHFUUBLWG } G_{\omega, \lambda}\left(s d_{1}, s d_{2}, \ldots, s d_{n}\right)=\frac{1}{\lambda}\left(\bigotimes_{j=1}^{n}\left(\lambda s d_{j}\right)^{\omega_{j}}\right) \\
& =\bigcup_{\left(\tilde{s}_{g_{j}}, \widetilde{h}_{j}, \tilde{g}_{j}\right) \in s d_{j}}\left(\left[s_{\left.(1 / \lambda) \prod_{j=1}^{n}\left(\lambda \Delta_{t_{0}}^{-1}\left(T F_{t_{0}}^{t_{j_{1}}}\left(\psi\left(s_{\alpha_{j}}\right)\right)\right)\right)^{\omega_{j}}, s_{(1 / \lambda) \prod_{j=1}^{n}\left(\lambda \Delta_{t_{0}}^{-1}\left(T F_{t_{0}}^{t_{2}}\left(\psi\left(s_{\beta_{j}}\right)\right)\right)\right)^{\omega_{j}}}\right],}\right.\right. \\
& \bigcup_{\left[\mu_{j}^{L}, \mu_{j}^{U}\right] \in \widetilde{h}_{j},\left[\nu_{j}^{L}, \nu_{j}^{U}\right] \in \tilde{g}_{j}}\left\{\left\{\left[1-\left(1-\prod_{j=1}^{n}\left(1-\left(1-\mu_{j}^{L}\right)^{\lambda}\right)^{\omega_{j}}\right)^{1 / \lambda}, 1-\left(1-\prod_{j=1}^{n}\left(1-\left(1-\mu_{j}^{U}\right)^{\lambda}\right)^{\omega_{j}}\right)^{1 / \lambda}\right]\right\},\right. \\
& \left.\left.\left\{\left[\left(1-\prod_{j=1}^{n}\left(1-\left(v_{j}^{L}\right)^{\lambda}\right)^{\omega_{j}}\right)^{1 / \lambda},\left(1-\prod_{j=1}^{n}\left(1-\left(\nu_{j}^{U}\right)^{\lambda}\right)^{\omega_{j}}\right)^{1 / \lambda}\right]\right\}\right\}\right) .
\end{aligned}
$$

Definition 11. For a collection of IVDHF_UUBLNs $s d_{j}(j=1$, $\ldots, n), s d_{\sigma(j)}$ be the $j$ th largest, $w=\left(w_{1}, w_{2}, \ldots, w_{n}\right)^{T}$ be the aggregation-associated weighting vector, $w_{j} \in[0,1], \sum_{j=1}^{n} w_{j}$ $=1, \lambda$ is a parameter such that $\lambda \in(0,+\infty), S^{n} \longrightarrow S$. Then, 
(1) Generalized IVDHFUUBL Ordered Weighted Average (GIVDHFUUBLOWA) Operator

$$
\begin{aligned}
& \text { GIVDHFUUBLOWA } A_{\omega, \lambda}\left(s d_{1}, s d_{2}, \ldots, s d_{n}\right)=\left(\bigoplus_{j=1}^{n}\left(w_{j} s d_{\sigma(j)}{ }^{\lambda}\right)\right)^{1 / \lambda} \\
& =\bigcup_{\left(\tilde{s}_{\sigma_{\sigma(j)}}, \tilde{h}_{\sigma(j)}, \tilde{g}_{\sigma(j)}\right) \in s d_{\sigma(j)}}\left(\left[s_{\left(\sum_{j=1}^{n} w_{j}\left(\Delta_{t_{0}}^{-1}\left(T F_{t_{0}}^{t_{j}}\left(\psi\left(s_{\alpha_{\sigma(j)}}\right)\right)\right)^{\lambda}\right)^{1 / \lambda}\right.}, s_{\left(\sum_{j=1}^{n} w_{j}\left(\Delta_{t_{0}}^{-1}\left(T F_{t_{0}}^{t_{j}}\left(\psi\left(s_{\beta_{\sigma(j)}}\right)\right)\right)^{\lambda}\right)^{1 / \lambda}\right.}\right],\right. \\
& \bigcup_{\left.\left[\mu_{\sigma(j)}^{L}, \mu_{\sigma(j)}^{U}\right] \in \tilde{h}_{\sigma(j),}, \nu_{\sigma(j)}^{L}, \nu_{\sigma(j)}^{U}\right] \in \tilde{g}_{\sigma(j)}}\left\{\left\{\left[\left(1-\prod_{j=1}^{n}\left(1-\left(\mu_{\sigma(j)}^{L}\right)^{\lambda}\right)^{w_{j}}\right)^{1 / \lambda},\left(1-\prod_{j=1}^{n}\left(1-\left(\mu_{\sigma(j)}^{U}\right)^{\lambda}\right)^{w_{j}}\right)^{1 / \lambda}\right]\right\},\right. \\
& \left.\left.\left\{\left[1-\left(1-\prod_{j=1}^{n}\left(1-\left(1-v_{\sigma(j)}^{L}\right)^{\lambda}\right)^{w_{j}}\right)^{1 / \lambda}, 1-\left(1-\prod_{j=1}^{n}\left(1-\left(1-v_{\sigma(j)}^{U}\right)^{\lambda}\right)^{w_{j}}\right)^{1 / \lambda}\right]\right\}\right\}\right)
\end{aligned}
$$

(2) Generalized IVDHFUUBL Ordered Weighted Geometric (GIVDHFUUBLOWG) Operator

$$
\begin{aligned}
& \operatorname{GIVDHFUUBLOWG}_{w, \lambda}\left(s d_{1}, s d_{2}, \ldots, s d_{n}\right)=\frac{1}{\lambda}\left(\bigotimes_{j=1}^{n}\left(\lambda s d_{\sigma(j)}\right)^{w_{j}}\right)
\end{aligned}
$$

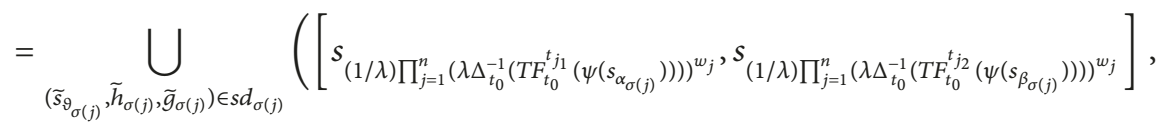

$$
\begin{aligned}
& \bigcup_{\left[\mu_{\sigma(j)}^{L}, \mu_{\sigma(j)}^{U}\right] \in \tilde{h}_{\sigma(j)},\left[\nu_{\sigma(j)}^{L}, \nu_{\sigma(j)}^{U}\right] \in \tilde{g}_{\sigma(j)}}\left\{\left\{\left[1-\left(1-\prod_{j=1}^{n}\left(1-\left(1-\mu_{\sigma(j)}^{L}\right)^{\lambda}\right)^{w_{j}}\right)^{1 / \lambda}, 1-\left(1-\prod_{j=1}^{n}\left(1-\left(1-\mu_{\sigma(j)}^{U}\right)^{\lambda}\right)^{w_{j}}\right)^{1 / \lambda}\right]\right\},\right. \\
& \left.\left.\left\{\left[\left(1-\prod_{j=1}^{n}\left(1-\left(v_{\sigma(j)}^{L}\right)^{\lambda}\right)^{\omega_{j}}\right)^{1 / \lambda},\left(1-\prod_{j=1}^{n}\left(1-\left(v_{\sigma(j)}^{U}\right)^{\lambda}\right)^{\omega_{j}}\right)^{1 / \lambda}\right]\right\}\right\}\right)
\end{aligned}
$$

Definition 12. For a collection of IVDHF_UUBLNs $s d_{j}(j=$ $1, \ldots, n), \omega=\left(\omega_{1}, \omega_{2}, \ldots, \omega_{n}\right)^{T}$ is the weighting vector, $\omega_{j} \in[0,1], \sum_{j=1}^{n} \omega_{j}=1 . n$ is a balancing coefficient. $w=\left(w_{1}, w_{2}, \ldots, w_{n}\right)^{T}$ is the aggregation-associated weighting vector with $w_{j} \in[0,1]$ and $\sum_{j=1}^{n} w_{j}=1$.
(1) Generalized IVDHFUUBL Hybrid Average (GIVDHFUUBLHA) Operator

$$
\begin{aligned}
& \text { GIVDHFUUBLHA } A_{\omega, w, \lambda}\left(s d_{1}, s d_{2}, \ldots, s d_{n}\right)=\left(\bigoplus_{j=1}^{n}\left(w_{j} \overline{s d}_{\sigma(j)}^{\lambda}\right)\right)^{1 / \lambda}
\end{aligned}
$$

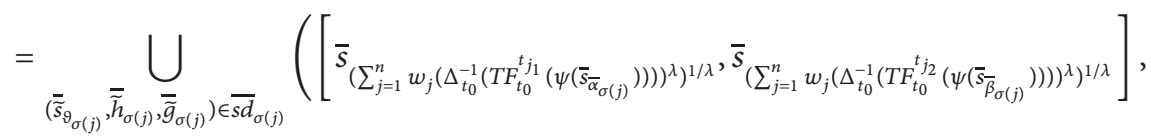




$$
\begin{gathered}
\bigcup_{\left.\left[\bar{\mu}_{\sigma(j)}^{L}, \bar{\mu}_{\sigma(j)}^{U}\right] \in \overline{\tilde{h}}_{\sigma(j)}, \bar{\nu}_{\sigma(j)}^{L}, \vec{v}_{\sigma(j)}^{U}\right] \in \overline{\tilde{g}}_{\sigma(j)}}\left\{\left\{\left[\left(1-\prod_{j=1}^{n}\left(1-\left(\bar{\mu}_{\sigma(j)}^{L}\right)^{\lambda}\right)^{w_{j}}\right)^{1 / \lambda},\left(1-\prod_{j=1}^{n}\left(1-\left(\bar{\mu}_{\sigma(j)}^{U}\right)^{\lambda}\right)^{w_{j}}\right)^{1 / \lambda}\right]\right\}\right. \\
\left.\left.\left\{\left[1-\left(1-\prod_{j=1}^{n}\left(1-\left(1-\bar{v}_{\sigma(j)}^{L}\right)^{\lambda}\right)^{w_{j}}\right)^{1 / \lambda}, 1-\left(1-\prod_{j=1}^{n}\left(1-\left(1-\bar{\nu}_{\sigma(j)}^{U}\right)^{\lambda}\right)^{w_{j}}\right)^{1 / \lambda}\right]\right\}\right\}\right)
\end{gathered}
$$

where $\overline{s d}_{\sigma(j)}$ is the $j$ th largest and

$$
\begin{gathered}
\overline{s d}_{j}=n \omega_{j} s d_{j}=\bigcup_{\left(\widetilde{s}_{\vartheta_{j}}, \widetilde{h}_{j}, \widetilde{g}_{j}\right) \in s d_{j}}\left(\left[s_{n \omega_{j}\left(\Delta_{t_{0}}^{-1}\left(T F_{t_{0}}^{t_{j_{1}}}\left(\psi\left(s_{\alpha_{j}}\right)\right)\right)\right.}, s_{n \omega_{j}\left(\Delta_{t_{0}}^{-1}\left(T F_{t_{0}}^{t_{j_{2}}}\left(\psi\left(s_{\beta_{j}}\right)\right)\right)\right)}\right],\right. \\
\left.\bigcup_{\left[\mu_{j}^{L}, \mu_{j}^{U}\right] \in \widetilde{h}_{j},\left[v_{j}^{L}, v_{j}^{U}\right] \in \widetilde{g}_{j}}\left\{\left\{\left[1-\left(1-\mu_{j}^{L}\right)^{n \omega_{j}}, 1-\left(1-\mu_{j}^{U}\right)^{n \omega_{j}}\right]\right\},\left\{\left[\left(\nu_{j}^{L}\right)^{n \omega_{j}},\left(\nu_{j}^{U}\right)^{n \omega_{j}}\right]\right\}\right\}\right) .
\end{gathered}
$$

\section{(2) Generalized IVDHFUUBL Hybrid Geometric}

\section{(GIVDHFUUBLHG) Operator}

$$
\begin{aligned}
& \operatorname{GIVDHFUUBLHG}_{w, \omega, \lambda}\left(s d_{1}, s d_{2}, \ldots, s d_{n}\right)=\frac{1}{\lambda}\left(\bigotimes_{j=1}^{n}\left(\lambda \overline{\overline{s d}}_{\sigma(j)}\right)^{w_{j}}\right)
\end{aligned}
$$

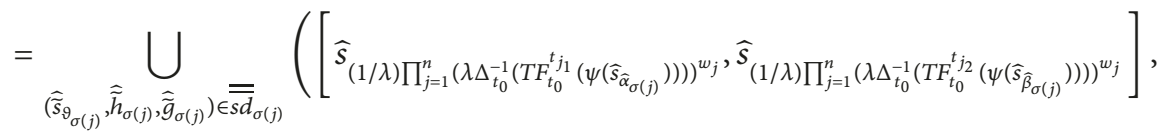

$$
\begin{aligned}
& \underset{\left[\hat{\mu}_{\sigma(j)}^{L}, \widehat{\mu}_{\sigma(j)}^{U}\right] \in \hat{\tilde{h}}_{\sigma(j)},\left[\hat{\nu}_{\sigma(j)}^{L}, \hat{\nu}_{\sigma(j)}^{U}\right] \in \hat{\tilde{g}}_{\sigma(j)}}{ }\left\{\left\{\left[1-\left(1-\prod_{j=1}^{n}\left(1-\left(1-\widehat{\mu}_{\sigma(j)}^{L}\right)^{\lambda}\right)^{w_{j}}\right)^{1 / \lambda}, 1-\left(1-\prod_{j=1}^{n}\left(1-\left(1-\widehat{\mu}_{\sigma(j)}^{U}\right)^{\lambda}\right)^{w_{j}}\right)^{1 / \lambda}\right]\right\},\right. \\
& \left.\left\{\left[\left(1-\prod_{j=1}^{n}\left(1-\left(\hat{v}_{\sigma(j)}^{L}\right)^{\lambda}\right)^{w_{j}}\right)^{1 / \lambda},\left(1-\prod_{j=1}^{n}\left(1-\left(\hat{v}_{\sigma(j)}^{U}\right)^{\lambda}\right)^{w_{j}}\right)^{1 / \lambda}\right]\right\}\right\}
\end{aligned}
$$

where $\overline{\overline{s d}}_{\sigma(j)}$ is the $j$ th largest and

$$
\begin{gathered}
\overline{\overline{s d}}_{j}=s d_{j}^{n \omega_{j}}=\bigcup_{\left(\widetilde{s}_{\vartheta_{j}}, \widetilde{h}_{j}, \widetilde{g}_{j}\right) \in s d_{j}}\left(\left[s_{\left(\Delta_{t_{0}}^{-1}\left(T T_{t_{0}}^{t_{j_{1}}}\left(\psi\left(s_{\alpha_{j}}\right)\right)\right)\right)^{n \omega_{j}}, s_{\left.\left(\Delta_{t_{0}}^{-1}\left(T F_{t_{0}}^{t_{j_{2}}}\left(\psi\left(s_{\beta_{j}}\right)\right)\right)\right)^{n \omega_{j}}\right],}}\right.\right. \\
\left.\bigcup_{\left[\mu_{j}^{L}, \mu_{j}^{U}\right] \in \widetilde{h}_{j},\left[v_{j}^{L}, v_{j}^{U}\right] \in \widetilde{g}_{j}}\left\{\left\{\left[\left(\mu_{j}^{L}\right)^{n \omega_{j}},\left(\mu_{j}^{U}\right)^{n \omega_{j}}\right]\right\},\left\{\left[1-\left(1-v_{j}^{L}\right)^{n \omega_{j}}, 1-\left(1-v_{j}^{U}\right)^{n \omega_{j}}\right]\right\}\right\}\right) .
\end{gathered}
$$


When confronted with ill-structured situations where decision-maker's complex attitudinal characters need to be included, order-inducing vectors provide an effective way [88-90]. Thus, we further define following induced operators for IVDHF_UUBLNs.

Definition 13. For a collection of IVDHF_UUBLNs $s d_{j}(j=$ $1, \ldots, n), \omega=\left(\omega_{1}, \omega_{2}, \ldots, \omega_{n}\right)^{T}$ is the weighting vector, $\omega_{j} \in[0,1], \sum_{j=1}^{n} \omega_{j}=1 . n$ is a balancing coefficient. $w=\left(w_{1}, w_{2}, \ldots, w_{n}\right)^{T}$ is the aggregation-associated weighting vector, $w_{j} \in[0,1]$ and $\sum_{j=1}^{n} w_{j}=1$. $\varepsilon_{j}$ denote a set of order inducing vectors.

(1) Induced Generalized IVDHFUUBL Hybrid Average (IGIVDHFUUBLHA) Operator

$$
\begin{aligned}
& \operatorname{I-GIVDHFUUBLHA} A_{\omega, w, \lambda}\left(<\varepsilon_{1}, s d_{1}>,<\varepsilon_{2}, s d_{2}>, \ldots,<\varepsilon_{n}, s d_{n}>\right)=\left(\bigoplus_{j=1}^{n}\left(w_{j} \bar{s}_{\pi(j)}^{\lambda}\right)\right)^{1 / \lambda} \\
& =\underbrace{}_{\left(\overline{\widetilde{s}}_{g_{\pi(j)}}\right.} \bigcup_{\left.\overline{\tilde{h}}_{\pi(j)}, \overline{\tilde{g}}_{\pi(j)}\right) \in \overline{s d}_{\pi(j)}}\left(\left[\bar{s}_{\left(\sum_{j=1}^{n} w_{j}\left(\Delta_{t_{0}}^{-1}\left(T T_{t_{0}}^{t_{j_{1}}}\left(\psi\left(\bar{s}_{\bar{\alpha}_{\pi(j)}}\right)\right)\right)\right)^{\lambda}\right)^{1 / \lambda}}, \bar{s}_{\left(\sum_{j=1}^{n} w_{j}\left(\Delta_{t_{0}}^{-1}\left(T F_{t_{0}}^{t}\left(\psi\left(\bar{s}_{\bar{\beta}_{\pi(j)}}\right)\right)\right)\right)^{\lambda}\right)^{1 / \lambda}}\right],\right. \\
& \bigcup_{\left[\bar{\mu}_{\pi(j)}^{L}, \bar{\mu}_{\pi(j)}^{U}\right] \in \overline{\tilde{h}}_{\pi(j)},\left[\bar{v}_{\pi(j)}^{L}, \bar{v}_{\pi(j)}^{U}\right] \in \overline{\tilde{g}}_{\pi(j)}}\left\{\left\{\left[\left(1-\prod_{j=1}^{n}\left(1-\left(\bar{\mu}_{\pi(j)}^{L}\right)^{\lambda}\right)^{w_{j}}\right)^{1 / \lambda},\left(1-\prod_{j=1}^{n}\left(1-\left(\bar{\mu}_{\pi(j)}^{U}\right)^{\lambda}\right)^{w_{j}}\right)^{1 / \lambda}\right]\right\},\right. \\
& \left.\left.\left\{\left[1-\left(1-\prod_{j=1}^{n}\left(1-\left(1-\bar{v}_{\pi(j)}^{L}\right)^{\lambda}\right)^{w_{j}}\right)^{1 / \lambda}, 1-\left(1-\prod_{j=1}^{n}\left(1-\left(1-\bar{v}_{\pi(j)}^{U}\right)^{\lambda}\right)^{w_{j}}\right)^{1 / \lambda}\right]\right\}\right\}\right),
\end{aligned}
$$

where $\left(\overline{s d}_{\pi(1)}, \overline{s d}_{\pi(2)}, \ldots, \overline{s d}_{\pi(n)}\right)$ is decreasing order of $\left(\overline{s d}_{1}\right.$, $\left.\overline{s d}_{2}, \ldots, \overline{s d}_{n}\right)$ according to the order inducing vector $\varepsilon_{j}$, and

$$
\begin{gathered}
\overline{s d}_{j}=n \omega_{j} s d_{j}=\bigcup_{\left(\widetilde{s}_{\vartheta_{j}}, \widetilde{h}_{j}, \widetilde{g}_{j}\right) \in s d_{j}}\left(\left[s_{n \omega_{j}\left(\Delta_{t_{0}}^{-1}\left(T F_{t_{0}}^{t_{j_{1}}}\left(\psi\left(s_{\alpha_{j}}\right)\right)\right)\right.}, s_{n \omega_{j}\left(\Delta_{t_{0}}^{-1}\left(T F_{t_{0}}^{t_{j}}\left(\psi\left(s_{\beta_{j}}\right)\right)\right)\right)}\right],\right. \\
\left.\bigcup_{\left[\mu_{j}^{L}, \mu_{j}^{U}\right] \in \widetilde{h}_{j},\left[\nu_{j}^{L}, \nu_{j}^{U}\right] \in \widetilde{g}_{j}}\left\{\left\{\left[1-\left(1-\mu_{j}^{L}\right)^{n \omega_{j}}, 1-\left(1-\mu_{j}^{U}\right)^{n \omega_{j}}\right]\right\},\left\{\left[\left(v_{j}^{L}\right)^{n \omega_{j}},\left(\nu_{j}^{U}\right)^{n \omega_{j}}\right]\right\}\right\}\right) .
\end{gathered}
$$

(2) Induced Generalized IVDHFUUBL Hybrid Geometric (I-GIVDHFUUBLHG) Operator

$$
\begin{aligned}
& I-G I V D H F U U B L H G_{w, \omega, \lambda}\left(<\varepsilon_{1}, s d_{1}>,<\varepsilon_{2}, s d_{2}>, \ldots,<\varepsilon_{n}, s d_{n}>\right)=\frac{1}{\lambda}\left(\bigotimes_{j=1}^{n}\left(\lambda \overline{\overline{s d}}_{\pi(j)}\right)^{w_{j}}\right)
\end{aligned}
$$

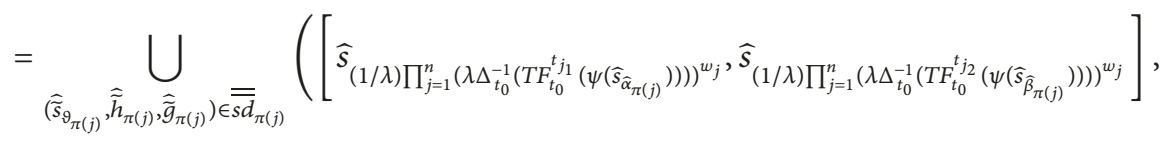

$$
\begin{aligned}
& \bigcup_{\left[\hat{\mu}_{\pi(j)}^{L}, \widehat{\mu}_{\pi(j)}^{U}\right] \in \widehat{\tilde{h}}_{\pi(j)},\left[\hat{\nu}_{\pi(j)}^{L}, \hat{\nu}_{\pi(j)}^{U}\right] \in \tilde{\tilde{g}}_{\pi(j)}}\left\{\left\{\left[1-\left(1-\prod_{j=1}^{n}\left(1-\left(1-\widehat{\mu}_{\pi(j)}^{L}\right)^{\lambda}\right)^{w_{j}}\right)^{1 / \lambda}, 1-\left(1-\prod_{j=1}^{n}\left(1-\left(1-\widehat{\mu}_{\pi(j)}^{U}\right)^{\lambda}\right)^{w_{j}}\right)^{1 / \lambda}\right]\right\},\right. \\
& \left.\left\{\left[\left(1-\prod_{j=1}^{n}\left(1-\left(\hat{v}_{\pi(j)}^{L}\right)^{\lambda}\right)^{w_{j}}\right)^{1 / \lambda},\left(1-\prod_{j=1}^{n}\left(1-\left(\widehat{v}_{\pi(j)}^{U}\right)^{\lambda}\right)^{w_{j}}\right)^{1 / \lambda}\right]\right\}\right\}
\end{aligned}
$$




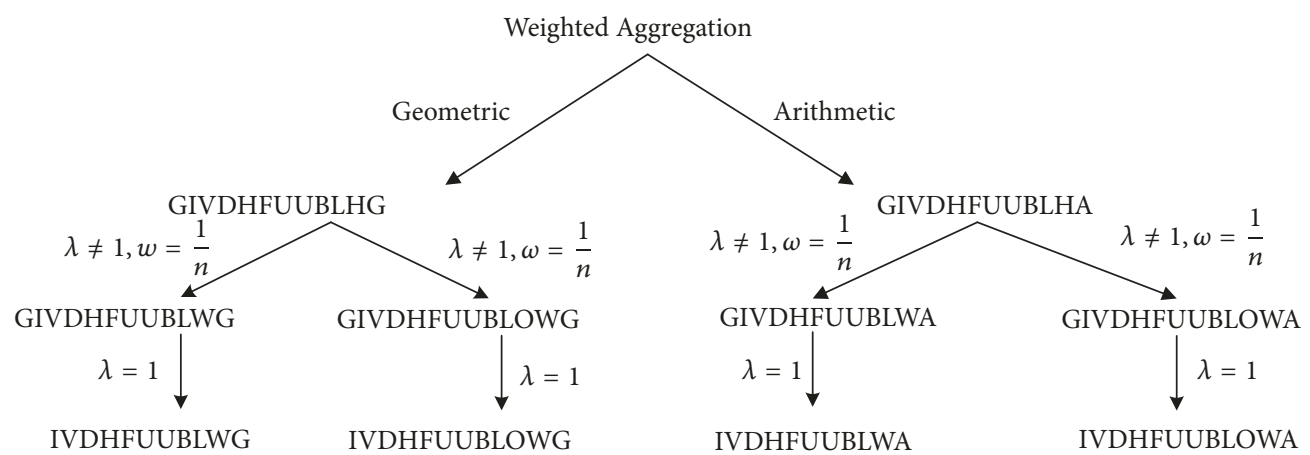

Figure 2

where $\left(\overline{\overline{s d}}_{\pi(1)}, \overline{\overline{s d}}_{\pi(2)}, \ldots, \overline{\overline{s d}}_{\pi(n)}\right)$ is decreasing order of $\left(\overline{\overline{s d}}_{1}\right.$, $\overline{\overline{s d}}_{2}, \ldots, \overline{\overline{s d}}_{n}$ ) according to the order inducing variables $\varepsilon_{j}$ and

$$
\begin{aligned}
& \overline{\overline{s d}}_{j}=s d_{j}^{n \omega_{j}}=\bigcup_{\left(\widetilde{s}_{g_{j}}, \widetilde{h}_{j}, \tilde{g}_{j}\right) \in s d_{j}}\left(\left[s_{\left(\Delta_{t_{0}}^{-1}\left(T_{t_{0}}^{t_{j_{1}}}\left(\psi\left(s_{\alpha_{j}}\right)\right)\right)^{n \omega_{j}}, s_{\left(\Delta_{t_{0}}^{-1}\left(T F_{t_{0}}^{t_{j}}\left(\psi\left(s_{\beta_{j}}\right)\right)\right)\right)^{n \omega_{j}}}\right],}\right.\right. \\
& \left.\bigcup_{\left[\mu_{j}^{L}, \mu_{j}^{U}\right] \in \widetilde{h}_{j},\left[\nu_{j}^{L}, \nu_{j}^{U}\right] \in \widetilde{g}_{j}}\left\{\left\{\left[\left(\mu_{j}^{L}\right)^{n \omega_{j}},\left(\mu_{j}^{U}\right)^{n \omega_{j}}\right]\right\},\left\{\left[1-\left(1-v_{j}^{L}\right)^{n \omega_{j}}, 1-\left(1-v_{j}^{U}\right)^{n \omega_{j}}\right]\right\}\right\}\right) .
\end{aligned}
$$

\subsection{Properties of the Proposed Generalized} Aggregation Operators

Theorem 14. With special values of $\lambda, w$ and $\omega$, the operators GIVDHFUUBLHA and GIVDHFUUBLHG can include a series of traditional aggregation operators as special cases and their relationship can be depicted in Figure 2.

Proof. See Appendix B.

As for the induced hybrid aggregation operators, we also have following theorem.

Theorem 15. If $\left(\overline{s d}_{\pi(1)}, \overline{s d}_{\pi(2)}, \ldots, \overline{s d}_{\pi(n)}\right)=\left(\overline{s d}_{\sigma(1)}, \overline{s d}_{\sigma(2)}\right.$, $\left.\ldots, \overline{s d}_{\sigma(n)}\right)$, I-GIVDHFUUBLHA reduces to GIVDHFUUBLHA; If $\left(\overline{\overline{s d}}_{\pi(1)}, \overline{\overline{s d}}_{\pi(2)}, \ldots, \overline{\overline{s d}}_{\pi(n)}\right)=\left(\overline{\overline{s d}}_{\sigma(1)}, \overline{\overline{s d}}_{\sigma(2)}, \ldots, \overline{\overline{s d}}_{\sigma(n)}\right)$, then I-GIVDHFUUBLHG reduces to the GIVDHFUUBLHG operator.

Theorem 16. All the proposed generalized operators GIVDHFUUBLWA, GIVDHFUUBLWG, GIVDHFUUBLOWA, GIVDHFUUBLOWG, GIVDHFUUBLHA, GIVDHFUUBLHG, I-GIVDHFUUBLHA, and I-GIVDHFUUBLHG hold the following properties: (1) Commutativity; (2) Idempotency; (3) Boundedness.

Based on above theorems, following properties can also be derived.
Theorem 17. For a collection of IVDHF_UUBLNs $s d_{j}=$ $\left(\widetilde{s}_{\vartheta_{j}}, \widetilde{h}_{j}, \widetilde{g}_{j}\right), \omega=\left(\omega_{1}, \omega_{2}, \ldots, \omega_{n}\right)^{T}$ is the weight vector of $s d_{j}$ with $\omega_{j} \in[0,1]$ and $\sum_{j=1}^{n} \omega_{j}=1, \lambda>0$; then we have

(1) IVDHFUUBLWG $G_{\omega}\left(s d_{1}, s d_{2}, \ldots, s d_{n}\right) \leq$ IVDHFUUBLWA ${ }_{\omega}\left(s d_{1}, s d_{2}, \ldots, s d_{n}\right)$;

(2) IVDHFUUBLWG $G_{\omega}\left(s d_{1}, s d_{2}, \ldots, s d_{n}\right) \leq$ GIVDHFUUBLWA $A_{\omega, \lambda}\left(s d_{1}, s d_{2}, \ldots, s d_{n}\right)$;

(3) GIVDHFUUBLWG $G_{\omega, \lambda}\left(s d_{1}, s d_{2}, \ldots, s d_{n}\right) \leq$ IVDHFUUBLWA $A_{\omega}\left(s d_{1}, s d_{2}, \ldots, s d_{n}\right)$.

Proof. Omitted for concision.

Theorem 18. For a collection of IVDHF_UUBLNs $s d_{j}=\left(\widetilde{s}_{\vartheta_{j}}\right.$, $\left.\widetilde{h}_{j}, \widetilde{g}_{j}\right), w=\left(w_{1}, w_{2}, \ldots, w_{n}\right)^{T}$ is the weighting vector of $s d_{j}$ with $w_{j} \in[0,1]$ and $\sum_{j=1}^{n} w_{j}=1, \lambda>0$. Then,

(1) IVDHFUUBLOWG $G_{w}\left(s d_{1}, s d_{2}, \ldots, s d_{n}\right) \leq$ IVDHFUUBLOWA $A_{w}\left(s d_{1}, s d_{2}, \ldots, s d_{n}\right)$;

(2) IVDHFUUBLOWG $G_{w}\left(s d_{1}, s d_{2}, \ldots, s d_{n}\right) \leq$ $\operatorname{GIVDHFUUBLOWA}_{w, \lambda}\left(s d_{1}, s d_{2}, \ldots, s d_{n}\right)$;

(3) GIVDHFUUBLOWG $G_{w, \lambda}\left(s d_{1}, s d_{2}, \ldots, s d_{n}\right) \leq$ IVDHFUUBLOWA $A_{w}\left(s d_{1}, s d_{2}, \ldots, s d_{n}\right)$.

Proof. Omitted for concision. 


\section{An Integrated MADM Approach for Tackling Complex Green Contractor Selection Problems}

As described in Section 2, we take the green contractor selection as a special type of complicate MADM problems that synthesizes three characteristics of decision hesitancy $[21,22]$, attributes interdependency [84], and group attitudinal characters [61]. Therefore, in this section, we construct an integrated MADM approach to tackle the complex green contractor selection problems. Suppose $X=$ $\left\{x_{1}, x_{2}, \ldots, x_{m}\right\}$ is the set of alternative green contractors and $A=\left\{A_{1}, A_{2}, \ldots, A_{n}\right\}$ is the set of evaluative attributes. $\omega=$ $\left(\omega_{1}, \omega_{2}, \ldots, \omega_{n}\right)^{T}$ is weighting vector for the attributes, $\omega_{i} \geq 0$, $\sum_{i=1}^{n} \omega_{i}=1$. Let $\widetilde{R}=\left(\widetilde{r}_{i j}\right)_{n \times m}$ denote the decision matrix in which $\widetilde{r}_{i j}=\left(\left[s_{\alpha_{i j}}, s_{\beta_{i j}}\right], \widetilde{h}_{i j}, \widetilde{g}_{i j}\right)$ is an IVDHF_UUBLN given by decision-makers for alternative contractor $x_{j}$ with respect to attribute $A_{i}$. According to the mechanism of pair-wise comparisons among attributes in the DEMATEL method [63, 64], the interdependency among attributes can be obtained as a matrix $\widetilde{Z}=\left(z_{i, k}\right)_{n \times n}$, where $z_{i, k}$ indicates the degree to which $A_{i}$ affects $A_{k}$. Subsequently, based on the IVDHFUUBLS and its operations, we now present detailed steps of our MADM approach as shown in following Algorithm I.

Algorithm I. Hesitant fuzzy linguistic MADM with attributes interdependency and decision-makers' group attitudinal characters.
Step 1. Determine argument-dependent weighting vector $\omega^{A D}$ according to attribute values by programming model developed in the following Section 5.1.

Step 2. Obtain the attribute-interdependences based weighting vector $\omega^{A I}$ by use of DEMATEL method described in the following Section 5.2.

Step 3. Calculate synthesized attribute weighting vector according to

$$
\omega=\alpha \omega^{A I}+\beta \omega^{A D}
$$

where $\alpha$ and $\beta$ are parameters to reflect decision characteristics of decision organizations, $0 \leq \alpha, \beta \leq 1, \alpha+\beta=$ 1.

Step 4. Check requirements for order inducing. If no additional order inducing required, then go to Step 5; otherwise go to Step 6.

Step 5. Utilize generalized aggregation operators to get the overall IVDHF_UUBLNs $\widetilde{r}_{j}(j=1, \ldots, m)$ for each alternative $x_{j}$. Here we take GIVDHFUUBLHA operator for example because it can include other traditional operators as its special cases. Therefore, we have

$$
\begin{aligned}
& \operatorname{GIVDHFUUBLHA}\left(\widetilde{r}_{1 j}, \widetilde{r}_{2 j}, \ldots, \widetilde{r}_{n j}\right)=\left(\bigoplus_{i=1}^{n}\left(w_{i}\left(\overline{\widetilde{r}}_{\sigma_{i} j}\right)^{\lambda}\right)\right)^{1 / \lambda}
\end{aligned}
$$

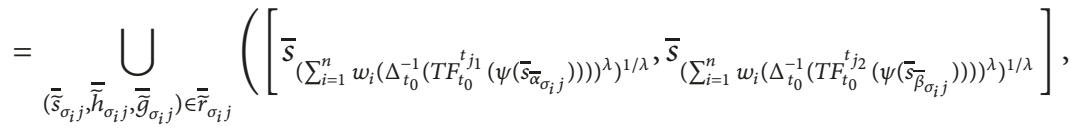

$$
\begin{aligned}
& \bigcup_{\left[\bar{\mu}_{\sigma_{i}}^{L}, \bar{\mu}_{\sigma_{i} j}^{U}\right] \in \overline{\tilde{h}}_{\sigma_{i} j},\left[\bar{v}_{\sigma_{i}}^{L}, \bar{\nu}_{\sigma_{i} j}^{U}\right] \in \overline{\tilde{g}}_{\sigma_{i} j}}\left\{\left\{\left[\left(1-\prod_{i=1}^{n}\left(1-\left(\bar{\mu}_{\sigma_{i} j}^{L}\right)^{\lambda}\right)^{w_{i}}\right)^{1 / \lambda},\left(1-\prod_{i=1}^{n}\left(1-\left(\bar{\mu}_{\sigma_{i} j}^{U}\right)^{\lambda}\right)^{w_{i}}\right)^{1 / \lambda}\right]\right\},\right. \\
& \left.\left.\left\{\left[1-\left(1-\prod_{i=1}^{n}\left(1-\left(1-\bar{\nu}_{\sigma_{i} j}^{L}\right)^{\lambda}\right)^{w_{i}}\right)^{1 / \lambda}, 1-\left(1-\prod_{i=1}^{n}\left(1-\left(1-\bar{\nu}_{\sigma_{i} j}^{U}\right)^{\lambda}\right)^{w_{i}}\right)^{1 / \lambda}\right]\right\}\right\}\right)
\end{aligned}
$$

where $\omega=\left(\omega_{1}, \omega_{2}, \ldots, \omega_{n}\right)^{T}$ is the weighting vector of $\widetilde{r}_{i j}$, $w=\left(w_{1}, w_{2}, \ldots, w_{n}\right)^{T}$ is the aggregation-associated weight vector, $\overline{\widetilde{r}}_{\sigma_{i} j}$ is the $i$ th largest of $\overline{\widetilde{r}}_{i j}$, and

$$
\begin{aligned}
& \overline{\widetilde{r}}_{i j}=n \omega_{i} \widetilde{r}_{i j}=\bigcup_{\left(\widetilde{s}_{i j}, \widetilde{h}_{i j}, \widetilde{g}_{i j}\right) \in \widetilde{r}_{i j}}\left(\left[s_{n \omega_{i}\left(\Delta_{t_{0}}^{-1}\left(T F_{t_{0}}^{t_{j 1}}\left(\psi\left(s_{\alpha_{i j}}\right)\right)\right)\right)}, s_{n \omega_{i}\left(\Delta_{t_{0}}^{-1}\left(T F_{t_{0}}^{t j_{2}}\left(\psi\left(s_{\beta_{i j}}\right)\right)\right)\right.}\right],\right. \\
& \left.\qquad \bigcup_{\left[\mu_{i j}^{L}, \mu_{i j}^{U}\right] \in \widetilde{h}_{i j},\left[\nu_{i j}^{L}, v_{i j}^{U}\right] \in \widetilde{\mathfrak{g}}_{i j}}\left\{\left\{\left[1-\left(1-\mu_{i j}^{L}\right)^{n \omega_{i}}, 1-\left(1-\mu_{i j}^{U}\right)^{n \omega_{i}}\right]\right\},\left\{\left[\left(v_{i j}^{L}\right)^{n \omega_{i}},\left(v_{i j}^{U}\right)^{n \omega_{i}}\right]\right\}\right\}\right) .
\end{aligned}
$$


Then go to Step 8.

Step 6. By use of the TOPSIS-based method developed in the following Section 5.3, we determine order-inducing vectors $\varepsilon$ to reflect decision-makers' group attitudinal characters.
Step 7. Utilizing induced generalized aggregation operators to get the overall IVDHF_UUBLNs $\tilde{r}_{j}(j=1,2, \ldots, m)$ for each alternative $x_{j}$, we have

$$
\begin{aligned}
& \operatorname{I-GIVDHFULHA}\left(\widetilde{r}_{1 j}, \widetilde{r}_{2 j}, \ldots, \widetilde{r}_{n m}\right)=\left(\bigoplus_{i=1}^{n}\left(w_{i}\left(\overline{\widetilde{r}}_{\pi_{i} j}\right)^{\lambda}\right)\right)^{1 / \lambda}
\end{aligned}
$$

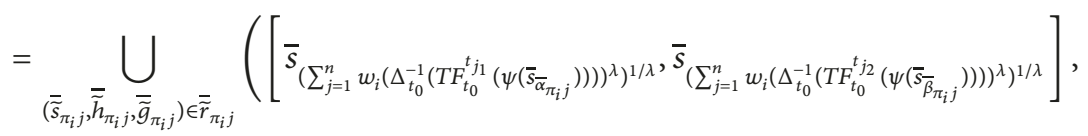

$$
\begin{aligned}
& \bigcup_{\left.\left[\bar{\mu}_{\pi_{i}}^{L}, \bar{\mu}_{\pi_{i} j}^{U}\right] \in \overline{\bar{h}}_{\pi_{i} j}, \bar{\nu} \bar{\nu}_{\pi_{i}}^{L}, \bar{\nu}_{\pi_{i} j}^{U}\right] \in \overline{\tilde{g}}_{\pi_{i} j}}\left\{\left\{\left[\left(1-\prod_{i=1}^{n}\left(1-\left(\bar{\mu}_{\pi_{i j}}^{L}\right)^{\lambda}\right)^{w_{i}}\right)^{1 / \lambda},\left(1-\prod_{i=1}^{n}\left(1-\left(\bar{\mu}_{\pi_{i} j}^{U}\right)^{\lambda}\right)^{w_{i}}\right)^{1 / \lambda}\right]\right\}\right. \text {, } \\
& \left.\left.\left\{\left[1-\left(1-\prod_{i=1}^{n}\left(1-\left(1-\bar{v}_{\pi_{i} j}^{L}\right)^{\lambda}\right)^{w_{i}}\right)^{1 / \lambda}, 1-\left(1-\prod_{i=1}^{n}\left(1-\left(1-\bar{\nu}_{\pi_{i} j}^{U}\right)^{\lambda}\right)^{w_{i}}\right)^{1 / \lambda}\right]\right\}\right\}\right),
\end{aligned}
$$

where $\omega=\left(\omega_{1}, \omega_{2}, \ldots, \omega_{n}\right)^{T}$ is the weighting vector of $\tilde{r}_{i j}, w=\left(w_{1}, w_{2}, \ldots, w_{n}\right)^{T}$ is the aggregation-associated weighting vector, $\overline{\widetilde{r}}_{\pi_{i} j}$ is the $i$ th largest of $\overline{\widetilde{r}}_{i j}$ reordered by the order-inducing vectors $\varepsilon$, and

$$
\begin{aligned}
& \overline{\widetilde{r}}_{i j}=n \omega_{i} \widetilde{r}_{i j}=\bigcup_{\left(\widetilde{s}_{i j}, \widetilde{h}_{i j}, \widetilde{q}_{i j}\right) \in \widetilde{r}_{i j}}\left(\left[s_{n \omega_{i}\left(\Delta_{t_{0}}^{-1}\left(T t_{t_{0}}^{t_{j 1}}\left(\psi\left(s_{\alpha_{i j}}\right)\right)\right) n \omega_{i} \alpha_{i j}\right.}, s_{n \omega_{i}\left(\Delta_{t_{0}}^{-1}\left(T F_{t_{0}}^{t_{j 1}}\left(\psi\left(s_{\beta_{i j}}\right)\right)\right)\right)}\right],\right. \\
& \left.\bigcup_{\left.\left[\mu_{i j}^{L}, \mu_{i j}^{U}\right] \in \tilde{h}_{i j}, \nu_{i j}^{L}, v_{i j}^{U}\right] \in \tilde{g}_{i j}}\left\{\left\{\left[1-\left(1-\mu_{i j}^{L}\right)^{n \omega_{i}}, 1-\left(1-\mu_{i j}^{U}\right)^{n \omega_{i}}\right]\right\},\left\{\left[\left(v_{i j}^{L}\right)^{n \omega_{i}},\left(v_{i j}^{U}\right)^{n \omega_{i}}\right]\right\}\right\}\right) .
\end{aligned}
$$

Step 8. Calculate scores $S\left(\widetilde{r}_{j}\right)$ and accuracy degrees $P\left(\widetilde{r}_{j}\right)$ for $\widetilde{r}_{j},(j=1,2, \ldots, m)$;

Step 9. According to Definition 5, output ranking order of alternative contractors.

In Algorithm I, to objectively derive unknown weighting vector for evaluative attributes, a synthesized attribute weighting vector is devised by fusing two parts of concern: (i) argument-dependent weighting vector and (ii) attributeinterdependences based weighting vector. The former is to reflect effects of attribute values on attribute weights, while the latter is to exploit effects of interdependences among attributes on their weights. For clarity, processing flowchart of Algorithm I is depicted in Figure 3.

\subsection{Determining Argument-Dependent Attribute Weighting} Vector $\omega^{A D}$. In order to derive attribute weighting vector appropriately from assessments, maximizing deviation method [91] is adopted here for its distinguishing ability and objectivity [92]. According to Wang [91], if performance values of each alternative have little differences under an attribute, it implicates such an attribute plays a less important role; otherwise, it lives a more important role in decisionmaking. Thus, if one attribute has similar values across alternatives, it should be assigned a smaller weight; contrariwise, it should be a bigger weight.

In light of the idea in maximizing deviation method, we develop a programming model to determine argumentdependent weighting vector $\omega^{A D}$. For attribute $A_{i}$, the standard deviation of alternative $x_{j}$ to all the other alternatives is denoted as

$$
\begin{aligned}
F_{i j}\left(\omega^{A D}\right)=\sum_{l=1}^{m} d\left(\widetilde{r}_{i j}, \widetilde{r}_{i l}\right) \omega_{i}^{A D}, \\
\quad i=1,2, \ldots, n ; j=1,2, \ldots, m .
\end{aligned}
$$

Let $F_{i}\left(\omega^{A D}\right)$ represent deviation value of all alternatives to other alternatives according to attribute $A_{i}$ and be formulated as follows: 


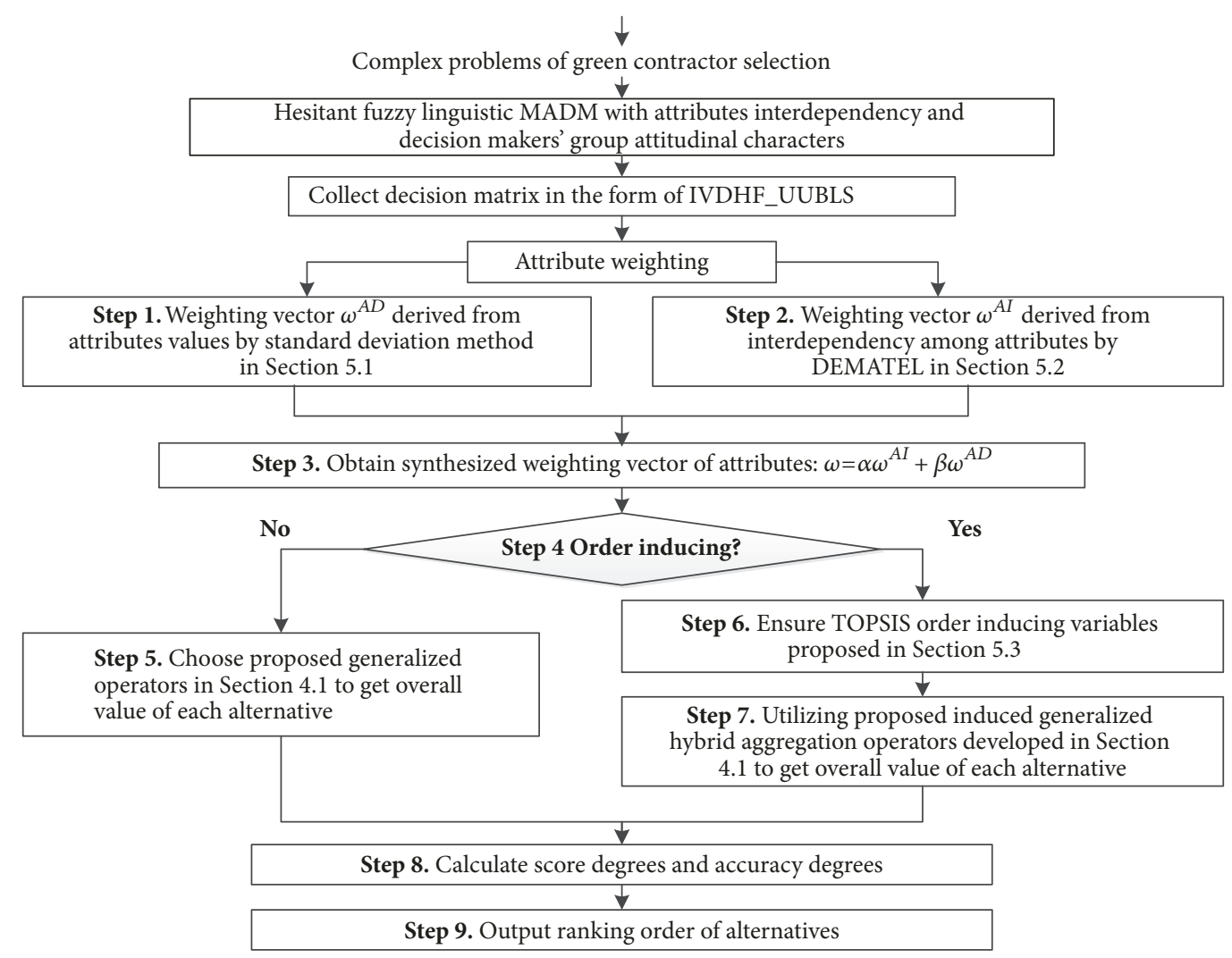

FIGURE 3: Processing flowchart of Algorithm I.

$$
\begin{aligned}
F_{i}\left(\omega^{A D}\right)=\sum_{j=1}^{m} F_{i j}\left(\omega^{A D}\right) & =\sum_{j=1}^{m} \sum_{l=1}^{m} d\left(\widetilde{r}_{i j}, \widetilde{r}_{i l}\right) \omega_{i}^{A D}, \\
i & =1,2, \ldots, n ; j=1,2, \ldots, m .
\end{aligned}
$$

Based on the analysis above, we can get following programming model (M-1) for obtaining optimal attribute weighting vector $\omega^{A D}$ :

$$
(M-1)\left\{\begin{array}{l}
\max \quad F\left(\omega^{A D}\right)=\sum_{i=1}^{n} F_{i}\left(\omega^{A D}\right)=\sum_{i=1}^{n} \sum_{j=1}^{m} \sum_{l=1}^{m} d\left(\widetilde{r}_{i j}, \widetilde{r}_{i l}\right) \omega_{i}^{A D} \\
\text { s.t. } \quad \sum_{i=1}^{n}\left(\omega_{i}^{A D}\right)^{2}=1, \quad \omega_{i}^{A D} \geq 0, i=1,2, \ldots, n,
\end{array}\right.
$$

where $d\left(\widetilde{r}_{i j}, \widetilde{r}_{i l}\right)$ can be calculated by Definition 6 .

To solve model (M-1), we can construct Lagrange function:

$$
\begin{aligned}
L\left(\omega_{i}^{A D}, \zeta\right)= & \sum_{i=1}^{n} \sum_{j=1}^{m} \sum_{l=1}^{m} d\left(\tilde{r}_{i j}, \widetilde{r}_{i l}\right) \omega_{i}^{A D} \\
& +\frac{\zeta}{2}\left(\sum_{i=1}^{n}\left(\omega_{i}^{A D}\right)^{2}-1\right),
\end{aligned}
$$

where $\zeta$ is the Lagrange multiplier.

Differentiate with respect to $\omega_{i}^{A D}(i=1,2, \ldots, m)$ and $\zeta$, and set these partial derivatives equal to zero; the following set of equations can be obtained:

$$
\begin{gathered}
\frac{\partial L}{\partial \omega_{i}^{A D}}=\sum_{j=1}^{m} \sum_{l=1}^{m} d\left(\tilde{r}_{i j}, \widetilde{r}_{i l}\right)+\zeta \omega_{i}^{A D}=0 \\
\frac{\partial L}{\partial \zeta}=\sum_{i=1}^{n}\left(\omega_{i}^{A D}\right)^{2}-1=0 .
\end{gathered}
$$

By normalizing the solutions of above equations, we can get a simple and exact formula for determining the attribute weights as follows:

$$
\omega_{i}^{A D}=\frac{\sum_{j=1}^{m} \sum_{l=1}^{m} d\left(\widetilde{r}_{i j}, \widetilde{r}_{i l}\right)}{\sum_{i=1}^{n} \sum_{j=1}^{m} \sum_{l=1}^{m} d\left(\widetilde{r}_{i j}, \widetilde{r}_{i l}\right)} .
$$
Weighting Vector $\omega^{A I}$. Some efforts [53, 60, 84, 93-95] 
have been made to address interdependencies between attributes in MADM. However, they unanimously cannot reveal the interdependences in a more complete way. While DEMATAEL technique $[63,96]$ gathers collective knowledge to capture influences between attributes more precisely and completely, so that weighting vector for attributes can be rationally determined according to strength measure of those influences $[64,97]$. Therefore, in tackling the complex MADM with interdependent attributes under IVDHF_UUBL environments, we present a DEMATEL-based method as the following Algorithm II to derive attribute weighting vector $\omega^{A I}$ from the viewpoint of attributes interdependency.

Algorithm II. DEMATEL-based method for ensuring weighting vector $\omega^{A I}$.

Step 1. Construct initial direct-influence matrix $Z=\left(z_{i k}\right)_{n \times n}$.

For attributes $A=\left\{A_{1}, A_{2}, \ldots, A_{n}\right\}$, decision-makers are asked to give direct effect that attribute $A_{i}$ has on attribute $A_{k}$, using a five-point scale with scores represented by natural language: (1) 0 - 'absolutely no influence'; (2) 1 - 'low influence'; (3) 2 - 'medium influence'; (4) $3-$ 'high influence'; (5) 4 - 'very high influence.' Then, the initial direct-influence matrix $Z=\left(z_{i k}\right)_{n \times n}$ can be established, with each element being the mean of same factors in different direct matrices.

Step 2. Calculate normalized direct-influence matrix $X=$ $\left(x_{i k}\right)_{n \times n}$. Using matrix $Z$, the normalized direct-influence matrix $X=\left(x_{i k}\right)_{n \times n}$ is calculated according to

$$
x_{i k}=\frac{z_{i k}}{s},
$$

where $s=\max \left\{\max _{1 \leq i \leq n} \sum_{k=1}^{n} z_{i k}, \max _{1 \leq k \leq n} \sum_{j=1}^{n} z_{i k}\right\}$. All elements in matrix $X$ are complying with $0 \leq x_{i k} \leq 1$, $0 \leq \sum_{k=1}^{n} x_{i k} \leq 1$, and $0 \leq \sum_{i=1}^{n} x_{i k} \leq 1$, and at least one row or column of the summation equals 1 .

Step 3. Derive total-influence matrix $T=\left(t_{i k}\right)_{n \times n}$. Based on matrix $X$, the total-influence matrix $T=\left(t_{i k}\right)_{n \times n}$ can be derived by summing the direct effects and all of the indirect effects,

$$
T=X+X^{2}+X^{3}+\cdots+X^{h}=X(I-X)^{-1},
$$

where $I$ represents identity matrix. When $h \longrightarrow \infty, X^{h}=$ $(0)_{n \times n}$.

Step 4. Compute influencing and influenced degrees of each attribute. The sum of rows and the sum of columns within the total-influence matrix $T$ are, respectively, expressed as the vectors $r$ and $c$ :

$$
\begin{aligned}
& r=\left(r_{i}\right)_{n \times 1}=\left(\sum_{k=1}^{n} t_{i k}\right)_{n \times 1}, \\
& c=\left(c_{k}\right)_{n \times 1}=\left(\sum_{j=1}^{n} t_{i k}\right)_{1 \times n}^{T},
\end{aligned}
$$

where $r_{i}$ denotes the sum of the $i$ th row in matrix $T$ and shows the sum of direct and indirect effects that attribute $A_{i}$ has on other attributes. Similarly, $c_{k}$ shows the sum of direct and indirect effects that attribute $A_{k}$ has received.

Step 5. Estimate weights of attributes based on influence degrees, according to

$$
\omega_{i}^{I *}=\left[\left(r_{i}+c_{i}\right)^{2}+\left(r_{i}-c_{i}\right)^{2}\right]^{1 / 2} .
$$

Then, the weighting vector $\omega^{A I}$ can be obtained by normalizing (33):

$$
\omega_{i}^{A I}=\frac{\omega_{i}^{I *}}{\sum_{i=1}^{n} \omega_{i}^{I *}} .
$$

5.3. Obtaining Order Inducing Variables $\varepsilon$ by TOPSIS-Based Method. For ill-defined MADM problems, induced aggregation operators $[89,90,98]$ provide an effective channel for experts to express complex attitudinal characters. The key step in induced aggregation operators is to obtain order-inducing vectors $\mathcal{E}$ which, however, are presumptively assigned in most papers [62]. Therefore, in this section, aiming at deducing the order-inducing vectors $\varepsilon$ rationally, we here devise a TOPSIS-based method in Algorithm III. Obviously, the Algorithm III is of generality for MADM with different forms of linguistic expression.

Algorithm III. TOPSIS-based method for obtaining order inducing vectors $\varepsilon$.

Step 1. Let the decision maker define IVDHF_UUBL ideal solution(s) $\widetilde{r}_{i}^{+}$and IVDHF_UUBL negative ideal solution(s) $\widetilde{r}_{i}$ corresponding to each attribute, in which

$$
\begin{aligned}
& \widetilde{r}_{i}^{+}=\left(\widetilde{r}_{i 1}^{+}, \widetilde{r}_{i 2}^{+}, \ldots, \widetilde{r}_{i p}^{+}\right), \\
& \widetilde{r}_{i}^{-}=\left(\widetilde{r}_{i 1}^{-}, \widetilde{r}_{i 2}^{-}, \ldots, \widetilde{r}_{i q}^{-}\right),
\end{aligned}
$$

where $p$ and $q$ denotes the number of $\widetilde{r}_{i}^{+}$and $\widetilde{r}_{i}^{-}$respectively.

Step 2. Calculate distances from each element in decision matrix to ideal solution(s) and negative solution(s) respectively:

$$
\begin{aligned}
& d_{i j}^{+}=\max _{l=1,2, \ldots, p} d\left(\widetilde{r}_{i j}, \widetilde{r}_{i l}^{+}\right), \\
& d_{i j}^{-}=\min _{l=1,2, \ldots, q} d\left(\widetilde{r}_{i j}, \widetilde{r}_{i l}\right),
\end{aligned}
$$

where $d\left(\widetilde{r}_{i j}, \widetilde{r}_{i l}^{+}\right)$and $d\left(\widetilde{r}_{i j}, \widetilde{r}_{i l}^{-}\right)$are calculated by the distance measure in Definition 6. If $p>1$, we choose the one with maximum distance; if $q>1$, we choose the one with minimum distance.

Step 3. Calculate coefficients according to

$$
c_{i j}=\frac{d_{i j}^{-}}{d_{i j}^{+}+d_{i j}^{-}}, \quad i=1,2, \ldots, n ; j=1,2, \ldots, m .
$$


TABLE 2: Decision matrix $\widetilde{R}$ in the form of IVDHF_UUBLS.

\begin{tabular}{|c|c|c|c|}
\hline & $x_{1}$ & $x_{2}$ & $x_{3}$ \\
\hline$A_{1}$ & $\begin{array}{c}([\mathrm{AH}, \mathrm{VH}],\{[0.2,0.3]\} \\
\{[0.2,0.4],[0.3,0.4]\}) \\
\end{array}$ & $\begin{array}{c}([\mathrm{H}, \mathrm{T}],\{[0.4,0.5] \\
[0.5,0.6]\},\{[0.2,0.3],[0.2,0.4]\})\end{array}$ & $\begin{array}{l}\text { ([AL, AH }],\{[0.1,0.2], \\
[0.1,0.3]\},\{[0.6,0.7]\}) \\
\end{array}$ \\
\hline$A_{2}$ & $\begin{array}{c}([\mathrm{M}, \mathrm{H}],\{[0.5,0.6]\} \\
\quad\{[0.2,0.3]\}) \\
\end{array}$ & $\begin{array}{c}([\mathrm{AH}, \mathrm{T}],\{[0.6,0.7]\} \\
\quad\{[0.1,0.2]\}) \\
\end{array}$ & $\begin{array}{r}([\mathrm{M}, \mathrm{VH}],\{[0.2,0.3]\} \\
\{[0.5,0.6],[0.6,0.7]\})\end{array}$ \\
\hline$A_{3}$ & $\begin{array}{c}([\mathrm{VL}, \mathrm{AL}],\{[0.3,0.4]\} \\
\{[0.4,0.5],[0.5,0.6]\})\end{array}$ & $\begin{array}{c}([\mathrm{M}, \mathrm{VH}],\{[0.6,0.7],[0.7,0.8]\} \\
\{[0.1,0.2]\})\end{array}$ & $\begin{array}{c}([\mathrm{VL}, \mathrm{L}],\{[0.4,0.5]\} \\
\{[0.2,0.3]\})\end{array}$ \\
\hline$A_{4}$ & $\begin{array}{c}([\mathrm{M}, \mathrm{AH}],\{[0.2,0.4]\} \\
\{[0.5,0.6]\})\end{array}$ & $\begin{array}{c}([\mathrm{L}, \mathrm{AL}],\{[0.3,0.5]\} \\
\{[0.2,0.3]\})\end{array}$ & $\begin{array}{l}([\mathrm{AL}, \mathrm{M}],\{[0.6,0.7]\} \\
\{[0.1,0.2],[0.2,0.3]\})\end{array}$ \\
\hline$A_{5}$ & $\begin{array}{l}\text { ([AL, M], }\{[0.4,0.5]\} \\
\{[0.1,0.2],[0.4,0.5]\}) \\
\end{array}$ & $\begin{array}{c}([\mathrm{AH}, \mathrm{H}],\{[0.6,0.7]\} \\
\{[0.1,0.2]\}) \\
\end{array}$ & $\begin{array}{l}([\mathrm{H}, \mathrm{VH}],\{[0.4,0.5] \\
[0.6,0.7]\},\{[0.1,0.3]\}) \\
\end{array}$ \\
\hline$A_{6}$ & $\begin{array}{c}([\mathrm{L}, \mathrm{M}],\{[0.1,0.2] \\
[0.3,0.5]\},\{[0.3,0.5]\})\end{array}$ & $\begin{array}{c}([\mathrm{VL}, \mathrm{L}],\{[0.2,0.4],[0.5,0.6]\} \\
\{[0.2,0.3]\})\end{array}$ & $\begin{array}{c}([\mathrm{AH}, \mathrm{H}],\{[0.3,0.4]\} \\
\{[0.4,0.5]\})\end{array}$ \\
\hline$A_{7}$ & $\begin{array}{c}([\mathrm{VL}, \mathrm{L}],\{[0.4,0.5] \\
[0.5,0.6]\},\{[0.2,0.3],[0.2,0.4]\})\end{array}$ & $\begin{array}{c}([\mathrm{M}, \mathrm{AH}],\{[0.5,0.6],[0.7,0.8]\} \\
\{[0.1,0.2]\})\end{array}$ & $\begin{array}{c}([\mathrm{AL}, \mathrm{M}],\{[0.4,0.5] \\
[0.5,0.6]\},\{[0.3,0.4]\})\end{array}$ \\
\hline$A_{8}$ & $\begin{array}{c}([\mathrm{AL}, \mathrm{AH}],\{[0.1,0.3]\} \\
\{[0.4,0.6]\})\end{array}$ & $\begin{array}{l}([\mathrm{AH}, \mathrm{H}],\{[0.5,0.7]\} \\
\{[0.1,0.2],[0.2,0.3]\})\end{array}$ & $\begin{array}{c}([\mathrm{AH}, \mathrm{H}],\{[0.4,0.6]\} \\
\{[0.3,0.4]\})\end{array}$ \\
\hline
\end{tabular}

TABLE 3: Direct influence matrix Z.

\begin{tabular}{|c|c|c|c|c|c|c|c|c|}
\hline & $A_{1}$ & $A_{2}$ & $A_{3}$ & $A_{4}$ & $A_{5}$ & $A_{6}$ & $A_{7}$ & $A_{8}$ \\
\hline$A_{1}$ & 0 & 3 & 4 & 1 & 3 & 1 & 2 & 4 \\
\hline$A_{2}$ & 1 & 0 & 2 & 4 & 4 & 2 & 3 & 1 \\
\hline$A_{3}$ & 4 & 2 & 0 & 1 & 3 & 2 & 4 & 1 \\
\hline$A_{4}$ & 2 & 3 & 1 & 0 & 3 & 4 & 1 & 2 \\
\hline$A_{5}$ & 4 & 2 & 1 & 3 & 0 & 1 & 4 & 3 \\
\hline$A_{6}$ & 2 & 4 & 3 & 4 & 4 & 0 & 4 & 2 \\
\hline$A_{7}$ & 4 & 1 & 2 & 3 & 3 & 1 & 0 & 4 \\
\hline$A_{8}$ & 1 & 2 & 4 & 3 & 3 & 2 & 4 & 0 \\
\hline
\end{tabular}

Step 4. Derive order inducing variable $\varepsilon_{i j}$ by descending order of $c_{i j}$. The greater the value $c_{i j}$, the bigger the $\varepsilon_{i j}$.

\section{Illustrative Example and Experiments}

6.1. Illustrative Case Study. Suppose a mega infrastructure project is selecting green contractor for one of its subprojects. A panel of decision-makers including managers, professors and competitive intelligence experts has been organized to comprehensively evaluate three alternative contractors (i.e., $x_{1}, x_{2}$ and $x_{3}$ ) under the eight attributes (i.e., $A_{1}-A_{8}$ ) listed in Section 2.

To elicit true decision preferences, we apply the IVDHF_UUBLS to assess the three alternatives $\left(x_{1}-x_{3}\right)$. Firstly, decision-makers are asked to vote on $x_{j}$ under each attribute $A_{i}$ using different unbalanced linguistic term sets $S_{1}$, $S_{2}$ and $S_{3}$, where $S_{1}=\{N, L, A L, M, A H, H, Q H, V H, A T, T\}$, $S_{2}=\{N, A N, V L, Q L, L, A L, M, A H, V H, T\}, S_{3}=\{N, V L$, $L, A M, M, Q M, A H, H, T\}$. In Figure 4, we show the adopted linguistic hierarchies and relationship between $S_{1}, S_{2}$ and $S_{3}$. Attributes $A_{1}, A_{2}, A_{5}$ and $A_{8}$ are evaluated by use of $S_{1}$, attributes $A_{3}, A_{4}$ and $A_{7}$ are evaluated by $S_{2}$, and attribute $A_{6}$ is evaluated by $S_{3}$. The highest-voted uncertain unbalanced linguistic term $\left[s_{m}, s_{n}\right]$ are identified. Secondly, the panel of decision-makers is asked to further express their opinions on membership degrees $\widetilde{h}(x)$ and nonmembership degrees $\widetilde{g}(x)$ to $\left[s_{m}, s_{n}\right]$. Then we can derive compound assessments (i.e., $\left.\left\langle x, \widetilde{s}_{\vartheta(x)}, \widetilde{h}(x), \widetilde{g}(x)\right\rangle\right)$ in the form of IVDHF_UUBLS and obtain the decision matrix $\widetilde{R}$ in Table 2. Additionally, in order to examine the interdependency among attributes, decision-makers are also required to assess the influential relations among the eight attributes, using DEMATEL-based Algorithm II. The direct influence matrix is collected and shown in Table 3.

Next, we apply the proposed Algorithm I to solve this green contractor selection problem. To derive the synthesized attribute weighting vector in Algorithm I, we assign $\alpha=\beta=0.5$. For scenarios with no group attitudinal characters, operator GIVDHFUUBLHA in Definition 12 is chosen. In contrast, for scenarios with group attitudinal characters, operator I-GIVDHFUUBLHA in Definition 13 will be chosen. Further, the panel of decision-makers is asked to determine their positive and negative ideal alternatives that are collected in Table 4, where corresponding orderinducing vectors that are derived from Algorithm III are also listed (see the rightmost column). Thereafter, operator I-GIVDHFUUBLHA in Definition 13 is utilized for aggregation. For GIVDHFUUBLHA and I-GIVDHFUUBLHA, we 
TABLE 4: Ideal alternatives $\left(r^{+}\right.$and $\left.r^{-}\right)$and derived order-inducing vectors $\varepsilon$.

\begin{tabular}{cccccr}
\hline & $r^{+}$ & $r^{-}$ & $x_{1}$ & $x_{2}$ & $x_{3}$ \\
\hline$A_{1}$ & $([\mathrm{VH}, \mathrm{T}],\{1\},\{0\})$ & $([\mathrm{N}, \mathrm{L}],\{1\},\{0\})$ & 5 & 6 & 6 \\
\hline$A_{2}$ & $([\mathrm{M}, \mathrm{AH}],\{[0.7,0.8]\},\{[0.1,0.2]\})$ & $([\mathrm{N}, \mathrm{L}],\{1\},\{0\})$ & 1 & 1 & 4 \\
\hline$A_{3}$ & $(\mathrm{~T},\{1\},\{0\})$ & $(\mathrm{L},\{1\},\{0\})$ & 8 & 7 & 8 \\
\hline$A_{4}$ & $([\mathrm{AL}, \mathrm{AH}],\{0.9\},\{0.1\})$ & $(\mathrm{T},\{1\},\{0\})$ & 2 & 2 & 1 \\
\hline$A_{5}$ & $([\mathrm{H}, \mathrm{T}],\{[0.9,1]\},\{0\})$ & $(\mathrm{N},\{1\},\{0\})$ & 7 & 5 & 5 \\
\hline$A_{6}$ & $(\mathrm{M},\{1\},\{0\})$ & $(\mathrm{N},\{1\},\{0\})$ & 6 & 3 & 3 \\
\hline$A_{7}$ & $(\mathrm{M},\{1\},\{0\})$ & $([\mathrm{N}, \mathrm{L}],\{1\},\{0\})$ & 3 & 8 & 7 \\
\hline$A_{8}$ & $([\mathrm{M}, \mathrm{AH}],\{1\},\{0\})$ & $(\mathrm{N},\{1\},\{0\})$ & 4 & 4 & 2 \\
\hline
\end{tabular}

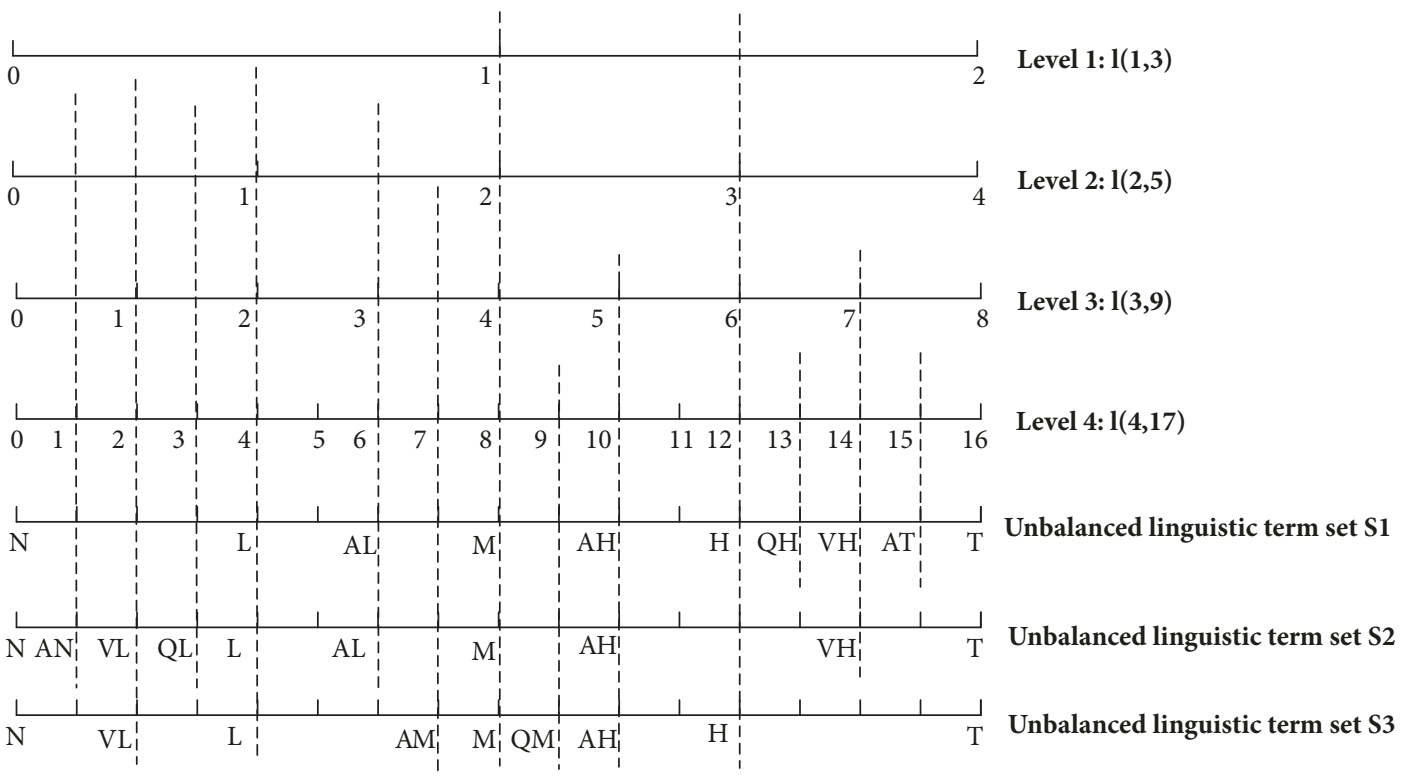

FIgURE 4: Unbalanced linguistic term sets $\left(S_{1}, S_{2}\right.$, and $\left.S_{3}\right)$ and their mapping in linguistic hierarchies.

assume $\lambda=2$, and $w=(0,0,0.15,0.25,0.25,0.25,0.1,0)^{T}$. Note that $w$ is a position weighting vector, which is derived by the fuzzy semantic quantitative operator [99]. After completing all steps in Algorithm I (see Appendix C), we obtain the ranking results and present them in Table 5. For the scores derived from Step 5, we find the ranking order is $x_{2}>x_{3}>x_{1}$, indicating that $x_{2}$ is the best solution. Step 7 gives the ranking order of $x_{2}>x_{1}>x_{3}$, indicating $x_{2}$ is still the most appropriate one, while $x_{1}$ is better than $x_{3}$ under the influence of $\varepsilon$.

6.2. Experiments on Ranking Results along with Various Parameter Configurations. In order to inspect the impacts of various parameter configurations on ranking results, in this section, more numerical examples are carried out. All eight operators that defined in Section 4.1 are applied to the above illustrative case, where the parameter $\lambda$ is configured as integers ranging from 1 to 20 and parameter $\alpha$ is configured to a value set of $\{0,0.3,0.5,0.8,0.9,1\}$.

Firstly, the four basic aggregation operators of GIVDHFUUBLWA, GIVDHFUUBLWG, GIVDHFUUBLOWA and
TABLE 5: Ranking results of operators GIVDHFUUBLHA and IGIVDHFUUBLHA.

\begin{tabular}{lc}
\hline Aggregation operator & Ranking order \\
\hline GIVDHFUUBLHA & $x_{2}>x_{3}>x_{1}$ \\
\hline I-GIVDHFUUBLHA & $x_{2}>x_{1}>x_{3}$ \\
\hline
\end{tabular}

GIVDHFUUBLOWG are applied to the former case. The same configuration $\alpha=\beta=0.5$ is chosen while $\lambda$ is tested as integers ranging from 1 to 20 . The ranking results derived from different parameter combinations have been collected in Table 6. As can be seen, four operators unanimously identify the contractor $x_{2}$ is the best one no matter how $\lambda$ changes. Regarding priority relation between $x_{1}$ and $x_{3}$, GIVDHFUUBLWA and GIVDHFUUBLOWA generate the same result of $x_{3}>x_{1}$; while results from GIVDHFUUBLWG and GIVDHFUUBLOWG got a change to $x_{1}>x_{3}$ at a certain value of parameter $\lambda$.

Actually, GIVDHFUUBLWA and GIVDHFUUBLOWA are generalized version of weighted arithmetic aggregation operators that produce more favorable results by emphasizing 
TABLE 6: Ranking orders obtained by GIVDHFUUBLWA, GIVDHFUUBLOWA, GIVDHFUUBLWG, and GIVDHFUUBLOWG along with $\lambda$.

\begin{tabular}{lcr}
\hline Operators & $\lambda$ (Integer) & Ranking order \\
\hline GIVDHFUUBLWA & {$[1,20]$} & $x_{2}>x_{3}>x_{1}$ \\
\hline GIVDHFUUBLOWA & {$[1,20]$} & $x_{2}>x_{3}>x_{1}$ \\
\hline GIVDHFUUBLWG & {$[1,2]$} & $x_{2}>x_{3}>x_{1}$ \\
& {$[3,20]$} & $x_{2}>x_{1}>x_{3}$ \\
\hline \multirow{2}{*}{ GIVDHFUUBLOWG } & {$[1,8]$} & $x_{2}>x_{3}>x_{1}$ \\
& {$[9,20]$} & $x_{2}>x_{1}>x_{3}$ \\
\hline
\end{tabular}

TABLE 7: Ranking results obtained by GIVDHFUUBLHA and I-GIVDHFUUBLHA along with $\alpha$, $\beta$, and $\lambda$.

\begin{tabular}{lccr}
\hline$\alpha, \beta$ & $\lambda$ (Integer) & GIVDHFUUBLHA & I-GIVDHFUUBLHA \\
\hline$\alpha=0, \beta=1$ & {$[1,20]$} & $x_{2}>x_{3}>x_{1}$ & $x_{2}>x_{1}>x_{3}$ \\
\hline$\alpha=0.3, \beta=0.7$ & {$[1,20]$} & $x_{2}>x_{3}>x_{1}$ & $x_{2}>x_{1}>x_{3}$ \\
\hline$\alpha=0.5, \beta=0.5$ & {$[1,20]$} & $x_{2}>x_{3}>x_{1}$ & $x_{2}>x_{1}>x_{3}$ \\
\hline$\alpha=0.8, \beta=0.2$ & {$[1,20]$} & $x_{2}>x_{3}>x_{1}$ & $x_{2}>x_{1}>x_{3}$ \\
\hline$\alpha=0.9, \beta=0.1$ & {$[1,20]$} & $x_{2}>x_{3}>x_{1}$ & $x_{2}>x_{1}>x_{3}$ \\
\hline$\alpha=1, \beta=0$ & {$[1,20]$} & $x_{2}>x_{3}>x_{1}$ & $x_{2}>x_{1}>x_{3}$ \\
\hline
\end{tabular}

TABLE 8: Ranking results obtained by GIVDHFUUBLHG and I-GIVDHFUUBLHG along with $\alpha$, $\beta$, and $\lambda$.

\begin{tabular}{lccc}
\hline$\alpha, \beta$ & $\lambda$ (Integer) & GIVDHFUUBLHG & I-GIVDHFUUBLHG \\
\hline$\alpha=0, \beta=1$ & {$[1,7]$} & $x_{2}>x_{3}>x_{1}$ & $x_{2}>x_{1}>x_{3}$ \\
& {$[8,20]$} & $x_{2}>x_{1}>x_{3}$ & $x_{2}>x_{1}>x_{3}$ \\
\hline$\alpha=0.3, \beta=0.7$ & {$[1,7]$} & $x_{2}>x_{3}>x_{1}$ & $x_{2}>x_{1}>x_{3}$ \\
& {$[8,20]$} & $x_{2}>x_{1}>x_{3}$ & $x_{2}>x_{1}>x_{3}$ \\
\hline$\alpha=0.5, \beta=0.5$ & {$[1,8]$} & $x_{2}>x_{3}>x_{1}$ & $x_{2}>x_{1}>x_{3}$ \\
\hline$\alpha=0.8, \beta=0.2$ & {$[9,20]$} & $x_{2}>x_{1}>x_{3}$ & $x_{2}>x_{1}>x_{3}$ \\
\hline$\alpha=0.9, \beta=0.1$ & {$[1,17]$} & $x_{2}>x_{3}>x_{1}$ & $x_{2}>x_{1}>x_{3}$ \\
\hline$\alpha=1, \beta=0$ & {$[18,20]$} & $x_{2}>x_{1}>x_{3}$ & $x_{2}>x_{1}>x_{3}$ \\
\hline & {$[1,20]$} & $x_{2}>x_{3}>x_{1}$ & $x_{2}>x_{1}>x_{3}$ \\
\hline
\end{tabular}

membership degrees to a uncertain linguistic term thus can be considered as optimistic operators, while GIVDHFUUBLWG and GIVDHFUUBLOWG are generalized geometric aggregation operators that produce more favorable results by emphasizing nonmembership degrees thus can be considered as pessimistic operators $[100,101]$. Therefore, the ranking results in Table 6 could be perceived as: for optimism-based decision-making, alternative $x_{3}$ always stands out better than $x_{1}$ when $\lambda$ gradually amplifies degree of optimism; but for pessimism-based decision-making, $x_{1}$ turns out better than $x_{3}$ when $\lambda$ gradually amplifies degree of pessimism. $\lambda$ can be considered as the parameter for amplifying degree of optimism or pessimism [101].

Next, to inspect the impacts of various combinations of $\omega^{A I}$ and $\omega^{A D}$ on ranking results, another four aggregation operators of GIVDHFUUBLHA, GIVDHFUUBLHG, IGIVDHFUUBLHA and I-GIVDHFUUBLHG also have been applied to the above illustrative case with varying $\alpha, \beta$ and $\lambda$.
All results have been collected in Tables 7 and 8 for comparison. As can be seen from Table 7, ranking results generated by operator GIVDHFUUBLHA keep the same as $x_{2}>x_{3}>x_{1}$ for all test combinations of parameters. However, as shown in Table 8, although GIVDHFUUBLHG is also capable of identifying $x_{2}$ as the best contractor, the priority relations between $x_{1}$ and $x_{3}$ hold similar changing patterns in ranking results in Table 6 that were output by GIVDHFUUBLWG and GIVDHFUUBLOWG. Regarding decision situations where objective attribute weighting vector $\omega^{A D}$ is only considered ( $\alpha$ $=0$ ) or its dominant importance (such as $\alpha=0.3$ ) is accepted, $x_{1}>x_{3}$ is a clear observation when parameter $\lambda$ is set to an integer in interval $[8,20]$. Contrariwise, under decision scenarios where subjective attribute weighting vector $\omega^{A I}$ is only needed $(\alpha=1)$ or its dominant importance (such as $\alpha=$ $0.9)$ is acknowledged, $x_{3}>x_{1}$ holds all the way $\lambda$ varies.

In order to more clearly show the score trajectories of alternative contractors along variations of parameter $\lambda$, we 


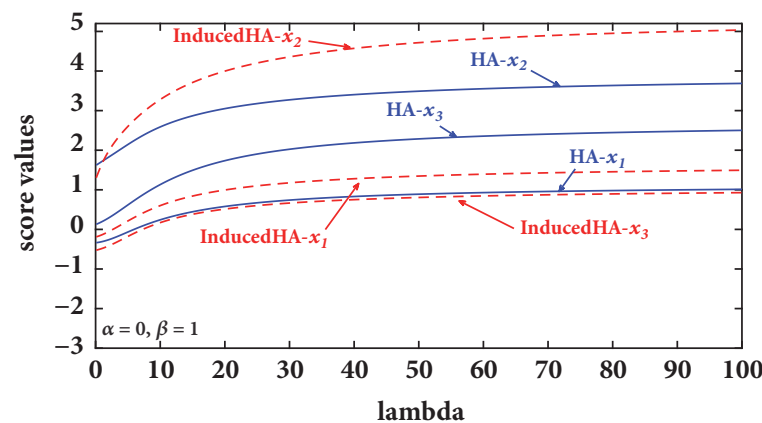

- HA

- - - InducedHA

(a) $\alpha=0$

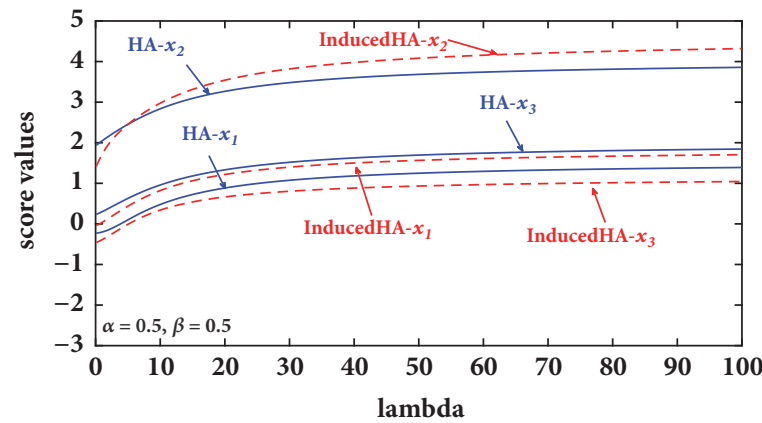

- HA

- - - InducedHA

(c) $\alpha=0.5$

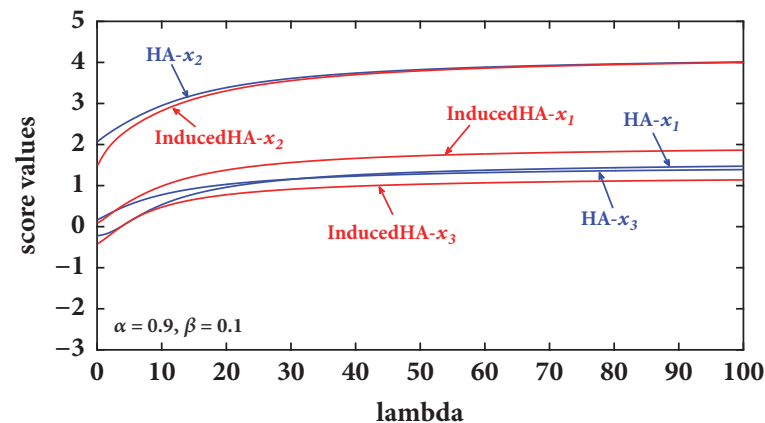

HA

- InducedHA

(e) $\alpha=0.9$

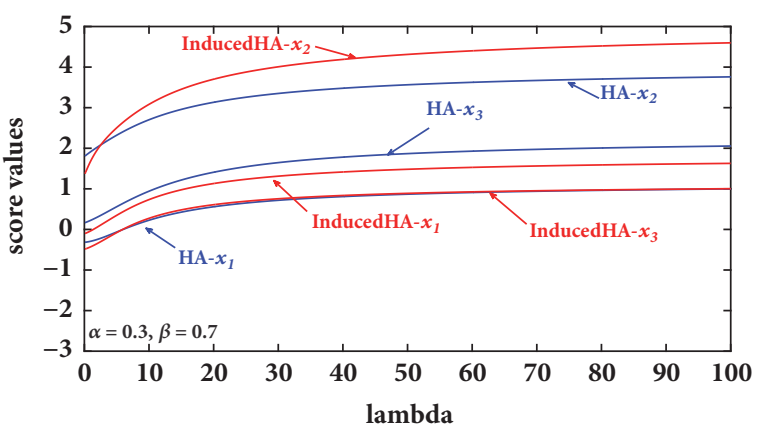

- HA

- - - InducedHA

(b) $\alpha=0.3$

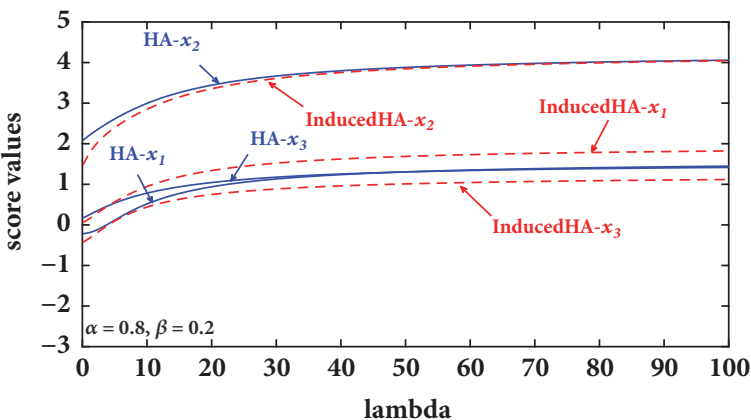

- HA

- - - InducedHA

(d) $\alpha=0.8$

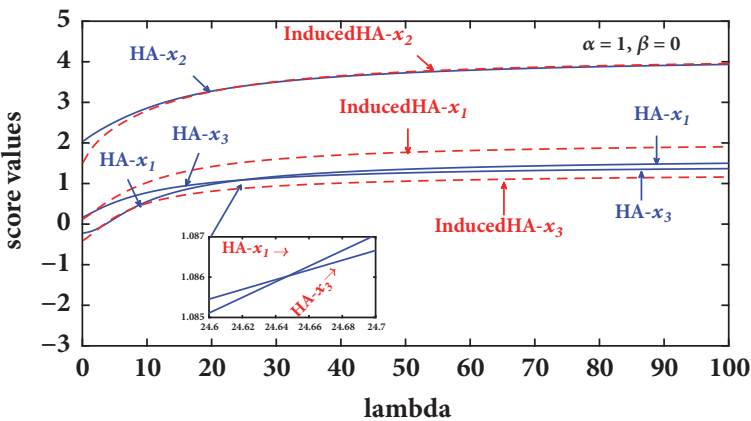

- HA

InducedHA

FIGURE 5: Score trajectories obtained by aggregation operators HA and InducedHA with different configuration of $\alpha$ : (a) $\alpha=0$, (b) $\alpha=0.3$, (c) $\alpha=0.5$, (d) $\alpha=0.7$, (e) $\alpha=0.9$, and (f) $\alpha=1$.

further configure parameter $\lambda$ to the value set of [0: 0.1:100] and conduct computational experiments to collect score values and ranking results (as shown in Figures 5 and 6) by four aggregation operators of GIVDHFUUBLHA, GIVDHFUUBLHG, I-GIVDHFUUBLHA and I-GIVDHFUUBLHG. For more clarity in Figures 5 and 6, please note that we use HA, HG, Induced-HA and Induced HG to respectively denote GIVDHFUUBLHA, GIVDHFUUBLHG, IGIVDHFUUBLHA and I-GIVDHFUUBLHG. In Figure 5, it can be observed that when dominant importance of $\omega^{A D}$ is emphasized ( $\alpha=0, \alpha=0.3, \alpha=0.5$ ), GIVDHFUUBLHA is capable of differentiating the three alternative contractors as same ranking order of $x_{2}>x_{3}>x_{1}$ for all $\lambda$; but with increases in $\alpha$, the margins between scores of $x_{1}$ and $x_{3}$ narrows and the rank order changes to $x_{1}>x_{3}$ when $\alpha$ gets 0.9 and 1. Interestingly in Figure 6, although GIVDHFUUBLHG is able to significantly differentiate alternative contractors $x_{1}$ and $x_{3}$ when dominant importance of $\omega^{A D}$ is emphasized ( $\alpha$ $=0, \alpha=0.3, \alpha=0.5$ ), rank order of $x_{1}$ and $x_{3}$ obviously changes once from $x_{3}>x_{1}$ to $x_{1}>x_{3}$ when $\lambda$ is bigger 


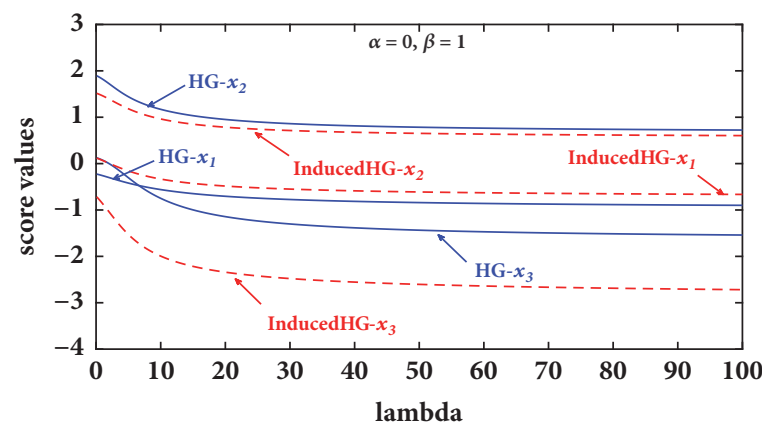

- HG

- - - InducedHG

(a) $\alpha=0$

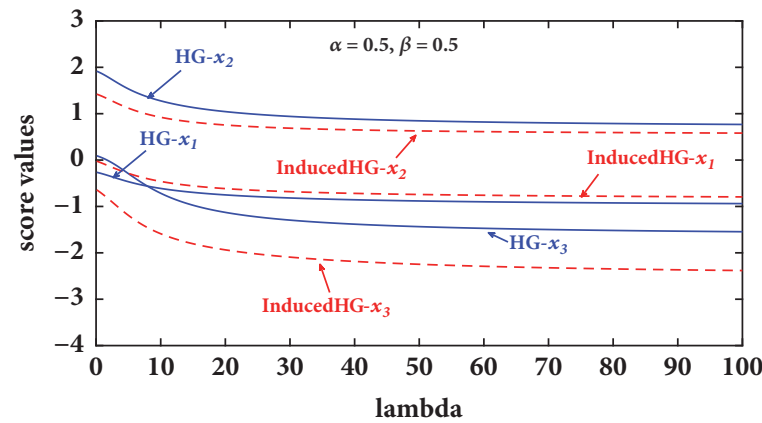

- HG

- - - InducedHG

(c) $\alpha=0.5$

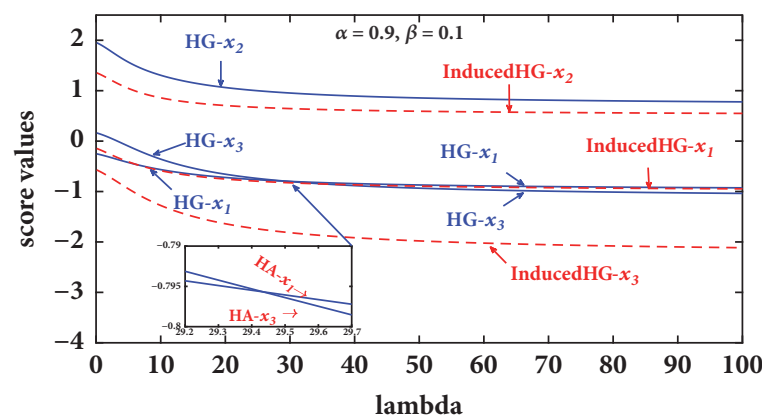

HG

InducedHG

(e) $\alpha=0.9$

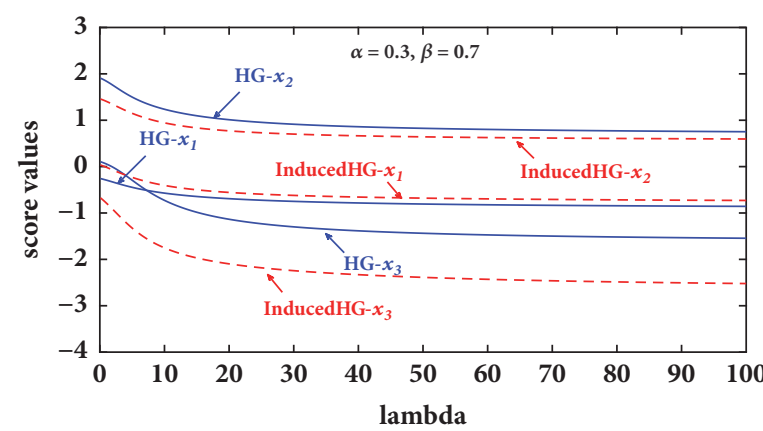

- HG

- - - InducedHG

(b) $\alpha=0.3$

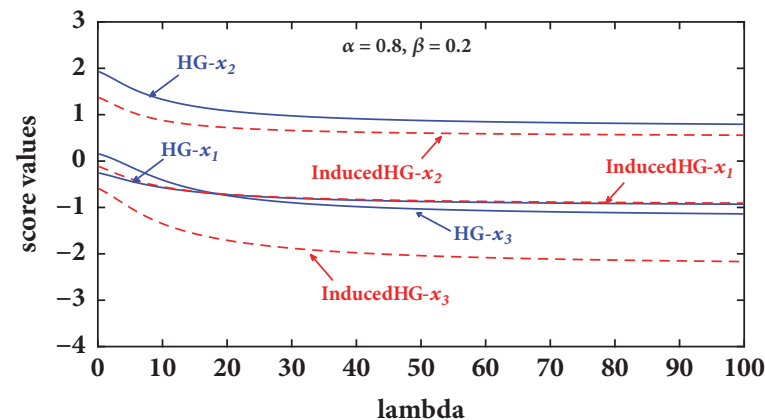

- HG

- - - InducedHG

(d) $\alpha=0.8$

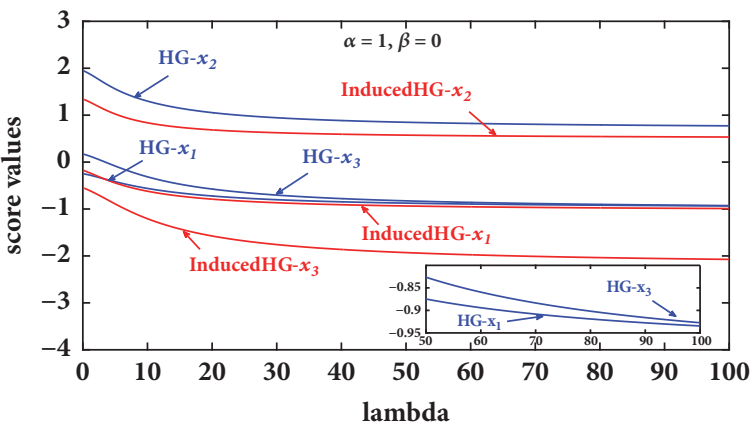

- HG

- . InducedHG

FIGURE 6: Score trajectories obtained by aggregation operators HG and InducedHG with different configuration of $\alpha$ : (a) $\alpha=0$, (b) $\alpha=0.3$, (c) $\alpha=0.5$, (d) $\alpha=0.7$, (e) $\alpha=0.9$, and (f) $\alpha=1$.

than 8 . When $\alpha$ increases to 1, GIVDHFUUBLHG maintains the rank order of $x_{3}>x_{1}$, but their score trajectories walk very close. Comparatively, I-GIVDHFUUBLHA and I-GIVDHFUUBLHG generate consistent ranking results of $x_{2}>x_{1}>x_{3}$ no matter $\lambda$ varies in all the above experiments.

In sum, when parameters are configured with typical values (i.e., $\alpha=0.5$ and $\lambda=1$ ) and no additional attitudinal information is taken into consideration, the proposed aggregation operators unanimously derive the same rank order $x_{2}>$ $x_{3}>x_{1}$ of for the three alternative contractors. However, with the amplification effect of parameter $\lambda$, analysis results from linear combination of $\omega^{A I}$ and $\omega^{A D}$ reveal alternative $x_{2}$ which consistently is the best choice but rank order of $x_{1}$ and $x_{3}$ changes because their score trajectories get close and crossed, which probably indicates that only decisionmaking information derived from the decision matrix is inadequate to significantly differentiate alternatives $x_{1}$ and $x_{3}$. While by integrating additional decision information about attitudinal characters of group experts, I-GIVDHFUUBLHA and I-GIVDHFUUBLHG manage to generate the identical 
TABLE 9: Order-inducing vectors obtained by our TOPSIS-based method for problem in [38].

\begin{tabular}{lcclr}
\hline & $G_{1}$ & $G_{2}$ & $G_{3}$ & $G_{4}$ \\
\hline$A_{1}$ & 0.2522 & 0.1629 & 0.2282 & 0.2714 \\
$A_{2}$ & 0.1 & 0.5243 & 0.1364 & 0.4689 \\
$A_{3}$ & 0.4081 & 0.1756 & 0.5244 & 0.3779 \\
$A_{4}$ & 0.4507 & 0.1409 & 0.1064 & 0.1322 \\
$A_{5}$ & 0.0697 & 0.2203 & 0.3753 & 0.1161 \\
\hline
\end{tabular}

TABLE 10: Order-inducing vectors obtained by our TOPSIS-based method for problem in [39].

\begin{tabular}{lcccc}
\hline & $G_{1}$ & $G_{2}$ & $G_{3}$ & $G_{4}$ \\
\hline$A_{1}$ & 0.4548 & 0.4969 & 0.4923 & 0.4732 \\
$A_{2}$ & 0.5092 & 0.47 & 0.4768 & 0.4645 \\
$A_{3}$ & 0.7192 & 0.4888 & 0.4953 & 0.4915 \\
$A_{4}$ & 0.6722 & 0.6163 & 0.5706 & 0.4929 \\
$A_{5}$ & 0.6104 & 0.4942 & 0.6463 & 0.4946 \\
\hline
\end{tabular}

TABLE 11: Ranking results obtained by applying different order-inducing vectors to $[38,39]$.

\begin{tabular}{lrr}
\hline Problems & Approaches & Ranking results \\
\hline Problem in [38] & IHFLOWA [38] + order-inducing vectors in [38] & $A_{3}>A_{1}>A_{2}>A_{5}>A_{4}$ \\
& IHFLOWA [38] + order-inducing vectors in Table 9 & $A_{3}>A_{1}>A_{2}>A_{4}>A_{5}$ \\
Problem in [39] & I-IVDHFLOWA [39] + order-inducing vectors in [39] & $A_{4}>A_{3}>A_{5}>A_{2}>A_{1}$ \\
& I-IVDHFLOWA [39] + order-inducing vectors in Table 10 & $A_{4}>A_{3}>A_{5}>A_{1}>A_{2}$ \\
\hline
\end{tabular}

permutation of $x_{2}>x_{1}>x_{3}$, which verifies that our proposed TOPSIS-based order-inducing approach is practical and useful in helping work out decision results more reasonably and robustly.

6.3. Application Studies of Proposed TOPSIS-Based OrderInducing Method. When confronting complex decisionmaking situations that need additional attitudinal characters of decision-makers, Yager and Filev [88] and Yager [102] introduced and encouraged to employ order-inducing vector to represent those attitudinal characters. Since then, the idea of order-inducing vector has been extended to various decision-making environments [98, 103], such as those based on intuitionistic fuzzy information [104, 105], linguistic information [106], hesitant fuzzy linguistic information [38], and interval-valued dual hesitant fuzzy uncertain linguistic information [39]. Generally, in existing literature, order-inducing vectors rely on experts to directly give [38, 39, 104-106].

In order to examine the adaptability of our TOPSISbased order-inducing method to linguistic decision-making environments, we respectively apply the method to resolve the same problems in references [38, 39]. Due to fact that there is no descriptions about how the order-inducing vectors were derived in both references [38, 39], we here apply extremums into our TOPSIS-based method to obtain orderinducing vectors, that is, $\left(s_{7},\{1\},\{0\}\right)$ and $\left(s_{1},\{0\},\{1\}\right)$ for [38], $\left(\left[s_{6}, s_{6}\right],\{1\},\{0\}\right)$, and $\left(\left[s_{0}, s_{0}\right],\{0\},\{1\}\right)$ for [39]. Then we obtain our order-inducing vectors listed in Table 9 for the problem adopted in [38], and our order-inducing vectors listed in Table 10 for [39].
Now, we apply the order-inducing vectors as shown in Tables 9 and 10 to resolve the problems in [38] and [39], respectively. Regarding the problem in [38], the score values of all alternatives are calculated as $S\left(A_{1}\right)=0.2602$, $S\left(A_{2}\right)=0.2417, S\left(A_{3}\right)=0.3577, S\left(A_{4}\right)=0.221$, and $S\left(A_{5}\right)=0.185$; so the corresponding ranking result is $A_{3}>A_{1}>A_{2}>$ $A_{4}>A_{5}$. As for the problem in [39], the score values of all alternatives are computed as $S\left(A_{1}\right)=3.9954, S\left(A_{2}\right)=3.783$, $S\left(A_{3}\right)=7.0827, S\left(A_{4}\right)=7.1792$, and $S\left(A_{5}\right)=5.813$; we thus obtain the ranking result $A_{4}>A_{3}>A_{5}>A_{1}>A_{2}$. For more clarity, brief descriptions of comparative approaches and obtained ranking results also have been put together in Table 11.

According to Table 11, the approaches that employ our order-inducing vectors derive the same ranking order of the better three alternatives for both comparative problems from $[38,39]$. As seen, our proposed TOPSIS-based orderinducing method exhibits effective and more understandable. Furthermore, when decision-makers are incapable of directly put forward all desired order-inducing vectors due to complexity in ill-structured problems, our method provides a feasible directions to obtain order-inducing vectors by figuring out ideal solutions. Comparatively, our proposed TOPSIS-based order-inducing method behaves effective and more operational in practical use.

6.4. Comparison with Decision-Making Approach Based on Degraded form of IVDHFULS. Note that our approach is intrinsically adaptive to decision situations based on degraded forms of IVDHF_UUBLS, such as the dual hesitant 
fuzzy linguistic sets introduced by Yang and Ju [54]. To further inspect feasibility of our approach, we here conduct comparative study on the same case adopted by Yang and Ju [54]. We firstly convert their original decision matrix into the form of IVDHF_UUBLS: crisp membership and nonmembership degrees are equivalently changed to intervals with same upper and lower limits (e.g., 0.2 turns out to be $[0.2,0.2])$, and linguistic variables are rewritten as uncertain linguistic variables (e.g., $s_{2}$ turns out to be $\left[s_{2}, s_{2}\right]$ ).

Since the case only got three attributes, among which interdependency could be not significant under general assumption, weighting vector $\omega^{A I}$ is not calculated. And for straight comparison, order inducing vector is not included either. Then, the argument-dependent attribute weighting vector can be obtained from Eq.(29) as $\omega^{A D}=$ $(0.3192,0.3132,0.3676)^{T}$. By use of Algorithm I with operator IVDHFUUBLWA (i.e., GIVDHFUUBLWA in case of $\lambda=1$, see Appendix B), we have the scores of alternatives: $s\left(A_{1}\right)=$ $0.3412, s\left(A_{2}\right)=0.1354, s\left(A_{3}\right)=0.3932, s\left(A_{4}\right)=0.7352$. Therefore, the ordering of alternatives is $A_{4}>A_{3}>A_{1}>A_{2}$, which is mainly in alignment with the ordering of $A_{4}>A_{1}>$ $A_{3}>A_{2}$ in Yang and Ju [54]. It is worth noticing that $\omega^{A D}$ used in our approach is derived objectively from assessments, while attribute weights $\omega^{*}=(0.35,0.25,0.40)^{T}$ used by Yang and Ju [54] were assigned empirically. Differences between $\omega^{A D}$ and $\omega^{*}$ lead to different ordering of $A_{1}$ and $A_{3}$. Comparatively speaking, our approach holds more adaptability and objectivity.

In general, our proposed MADM approach in this paper exhibits an effective way to tackle complex green contractor selection problems, in which subjective attributes weighting vector (such as $\omega^{A I}$ ) and objective attributes weighting vector (such as $\omega^{A D}$ ) exist. To comprehensively utilize these two fundamental facets of attributes weighting information, our case study and computational experiments suggest that linear combination method (i.e., $\omega=\alpha \omega^{A I}+\beta \omega^{A D}$ ) is effective to reflect effects of $\omega^{A I}$ and $\omega^{A D}$ by configuring parameter $\alpha$ or $\beta$. When there is no specific expert opinions to specify or hard to obtain, we suggest that parameter $\alpha$ chooses the value set of $\{0,0.2,0.5,0.8,1\}$. Furthermore, generalized aggregation operators are suggested to inspect score trajectories of alternative contractors along increasing of the operators' amplification parameter $\lambda$. If some alternative contractors' score values get very close or crossed so that they cannot be differentiated from each other significantly, we suggest to ask the panel of experts to derive opinions of ideal solutions and utilize our TOPSIS-based order-inducing method to include those expert opinions as attitudinal decision information, thereby obtaining more rational and robust decision results.

\section{Conclusions}

Aiming at tackling complex decision-making problems of green contractor selection that often hold simultaneously the characteristics of decision hesitancy, attributes interdependency, and group complex attitudinal characters, we have developed an effective IVDHF_UUBLS-based approach.
We utilize IVDHF_UUBLS to elicit complicate decisionmakers' assessments more objectively and comprehensively, we employ DEMATEL for addressing attribute interdependency more precisely, and we determine group attitudinal characters as order-inducing vectors based on ideal attitudes of experts rather than by empirical assignment. The main advantages of our approach are (i). IVDHF_UUBLS offers an adequate way to depict decision hesitancy; (ii). the designed distance measure for IVDHF_UUBLS avoids information distortion of traditional ones, which artificially add mismatching membership or nonmembership degrees; (iii). the developed generalized operators include traditional ones as special cases so as to provide flexibility in MADM based on IVDHF_UUBLS; (iv). the devised synthesized attribute weighting scheme provides an adaptive way to consider two necessary aspects: attributes interdependency-based weights and argument-dependent weights; (v). the TOPSIS-based method can deduce reasonable order-inducing vectors rather than empirical ones and holds generality in other linguistic decision environments. Results of case studies and experiments have verified the validity of the proposed approach.

When multiple decision organizations are involved, group decision-making based on IVDHF_UUBLS is an important research direction. Besides inducing preferences, for decision-making problems of high uncertainty, interactive mechanisms should also be studied to extract preferences. In addition, empirical studies of the proposed approach can be carried out to various areas, such as investment evaluation project management and vehicle selection in fleet operations.

\section{Appendix}

\section{A. Basic Notions for IVDHFS and Unbalanced Linguistic Term Set}

Definition A.1 (see [47]). Letting $X$ be a fixed set, then an IVDHFS on $X$ is defined as

$$
\widetilde{D}=\{\langle x, \widetilde{h}(x), \widetilde{g}(x)\rangle \mid x \in X\},
$$

where $\widetilde{h}(x)=\bigcup_{\left[\mu^{L}, \mu^{U}\right] \in \tilde{h}(x)}\{\widetilde{\mu}\}=\bigcup_{\left[\mu^{L}, \mu^{U}\right] \in \widetilde{h}(x)}\left\{\left[\mu^{L}, \mu^{U}\right]\right\}$ and $\tilde{g}(x)=\bigcup_{\left[\nu^{L}, \nu^{U}\right] \in \tilde{g}(x)}\{\tilde{\nu}\}=\bigcup_{\left[\nu^{L}, \nu^{U}\right] \in \tilde{g}(x)}\left\{\left[\nu^{L}, \nu^{U}\right]\right\}$ are two sets of interval values in $[0,1]$, denoting possible membership and non-membership degrees of element $x \in X$ to the set $\widetilde{D}$, respectively; with conditions: $\widetilde{\mu}, \widetilde{\nu} \in[0,1]$ and $0 \leq\left(\mu^{U}\right)^{+}+\left(\nu^{U}\right)^{+} \leq 1$; and for all $x \in X,\left(\mu^{U}\right)^{+} \epsilon$ $\widetilde{h}^{+}(x)=\bigcup_{\left[\mu^{L}, \mu^{U}\right] \in \widetilde{h}(x)} \max \left\{\mu^{U}\right\}$ and $\left(\nu^{U}\right)^{+} \in \tilde{g}^{+}(x)=$ $\bigcup_{\left[v^{L}, v^{U}\right] \in \tilde{g}(x)} \max \left\{v^{U}\right\}$.

For convenience, normally $\widetilde{d}=\{\widetilde{h}, \widetilde{g}\}$ is called an intervalvalued dual hesitant fuzzy element (IVDHFE), and $\widetilde{D}$ is the set of all IVDHFEs.

Definition A.2 (see [107]). Suppose $S=\left\{s_{j} \mid j=0,1, \ldots, g-\right.$ $1\}$ is a finite and totally ordered discrete linguistic term set, where $s_{j}$ represent possible values for a linguistic variable and $g$ is an odd cardinality. $S$ is uniformly and symmetrically distributed if the following two conditions are satisfied: (1) 
there exists a unique constant $\lambda>0$ such that $N S\left(s_{i}\right)-$ $N S\left(s_{j}\right)=l(i-j)$ for all $i, j=0,1, \ldots ., g-1$; (2) Let $S_{R}=$ $\left\{s \mid s \in S, s>S_{C}\right\}$ and $S_{L}=\left\{s \mid s \in S, s<S_{C}\right\}$. Let $\mathbb{C}\left(S_{R}\right)$ and $\mathbb{C}\left(S_{L}\right)$ be the cardinality of $S_{R}$ and $S_{L}$, then $\mathbb{C}\left(S_{R}\right)=\mathbb{C}\left(S_{L}\right)$. If $S$ is uniformly and symmetrically distributed, then $S$ is called a balanced linguistic term set. Otherwise, $S$ is called an unbalanced linguistic term set.

The following 2-tuple fuzzy linguistic representation model extends traditional linguistic term set to a continuous case so as to facilitate computing with linguistic variables.

Definition $A .3$ (see [59]). Let $S=\left\{s_{0}, s_{1}, \ldots, s_{g-1}\right\}$ be a linguistic term set and $\beta \in[0, g]$. Then a 2-tuple fuzzy linguistic variable expresses the equivalent information to $\beta$ is defined as

$$
\begin{aligned}
& \Delta:[0, g] \longrightarrow S \times[-0.5,0.5), \\
& \Delta(\beta)=\left(s_{i}, \alpha\right), \\
& \text { with } \begin{cases}s_{i}, & i=\operatorname{round}(\beta) \\
\alpha=\beta-i, & \alpha \in[-0.5,0.5),\end{cases} \\
& \Delta^{-1}\left(s_{i}, \alpha\right)=i+\alpha=\beta,
\end{aligned}
$$

where round(.) is the usual rounding operation and $\alpha$ is called symbolic translation.

To obtain 2-tuple fuzzy linguistic representations of unbalanced linguistic terms, the concept of linguistic hierarchies, i.e., $L H=\bigcup_{t} l(t, n(t))$, is used. $l(t, n(t))$ is a linguistic hierarchy with $t$ indicating the level of hierarchy and $n(t)$ denotes the granularity of the linguistic term set of $t$. Herrera, et al. [59] defined the following transformation functions between labels from different levels in multigranular linguistic information contexts without loss of information.

Definition A.4 (see [59]). In linguistic hierarchies $L H=$ $\bigcup_{t} l(t, n(t))$ whose linguistic term sets are represented by $S^{n(t)}=\left\{s_{0}^{n(t)}, \ldots, s_{n(t)-1}^{n(t)}\right\}$, the transformation function from a linguistic label in level $t$ to a label in consecutive level $t^{\prime}$ is defined as $T F_{t^{\prime}}^{t}: l(t, n(t)) \longrightarrow l\left(t^{\prime}, n\left(t^{\prime}\right)\right)$ such that

$$
\begin{aligned}
& \operatorname{TF}_{t^{\prime}}^{t}\left(s_{i}^{n(t)}, \alpha^{n(t)}\right) \\
& \quad=\Delta_{t^{\prime}}\left(\frac{\Delta_{t}^{-1}\left(s_{i}^{n(t)}, \alpha^{n(t)}\right)\left(n\left(t^{\prime}\right)-1\right)}{n(t)-1}\right) .
\end{aligned}
$$

By use of the above transformation function, any 2-tuple linguistic representation can be transformed into a term in $L H$. Detailed transformation procedures are listed as follows.
(1) Representation in linguistic hierarchy. To transform the unbalanced terms of $S$ into the corresponding terms in the $L H$, transformation function $\psi$ is employed to associate each unbalanced linguistic 2-tuple $\left(s_{i}, \alpha\right)$ with its linguistic 2tuple in $L H(\bar{S})$, i.e.,

$$
\psi: \bar{S} \longrightarrow L H(\bar{S})
$$

so that $\psi\left(s_{i}, \alpha\right)=\left(s_{I(i)}^{G(i)}, \lambda\right)$, for $\forall\left(s_{i}, \alpha\right) \in \bar{S}$.

(2) Computational phase. Firstly, transform $\left(s_{I(i)}^{G(i)}, \lambda\right)$ into linguistic 2-tuples, denoted as $\left(s_{I^{\prime}(i)}^{n\left(t^{\prime}\right)}, \lambda^{\prime}\right)$ in $\bar{S}^{n\left(t^{\prime}\right)}$, where

$$
\begin{aligned}
\left(s_{I^{\prime}(i)}^{n\left(t^{\prime}\right)}, \lambda^{\prime}\right) & =\operatorname{TF}\left(s_{I(i)}^{G(i)}, \lambda\right) \\
& =\Delta\left(\frac{\Delta^{-1}\left(s_{I(i)}^{G(i)}, \lambda\right) \cdot\left(n\left(t^{\prime}\right)-1\right)}{G(i)-1}\right) .
\end{aligned}
$$

Then, a computational model is used over $\bar{S}^{n\left(t^{\prime}\right)}$ with a result denoted as $\left(s_{r}^{n\left(t^{\prime}\right)}, \lambda_{r}\right) \in \bar{S}^{n\left(t^{\prime}\right)}$.

(3) Retranslation process. The result $\left(s_{r}^{n\left(t^{\prime}\right)}, \lambda_{r}\right) \in \bar{S}^{n\left(t^{\prime}\right)}$ is transformed into the unbalanced term in $\bar{S}$, by using the transformation function $\psi^{-1}$, i.e.,

$$
\psi^{-1}: L H(\bar{S}) \longrightarrow \bar{S}
$$

so that $\psi^{-1}\left(s_{r}^{n\left(t^{\prime}\right)}, \lambda_{r}\right)=\left(s_{\text {result }}, \lambda_{\text {result }}\right) \in \bar{S}$.

\section{B. Details of Theorem 14}

Proof. Given a collection of IVDHF_UUBLNs $s d_{j}=$ $\left(\widetilde{s}_{\vartheta_{j}}, \widetilde{h}_{j}, \widetilde{g}_{j}\right), \omega=\left(\omega_{1}, \omega_{2}, \ldots, \omega_{n}\right)^{T}$ is the weighting vector for $s d_{j}$ and $w=\left(w_{1}, w_{2}, \ldots, w_{n}\right)^{T}$ is the aggregation-associated weighting vector, $\lambda>0$, then,

(1) If $\lambda \neq 1$

(1) If $w=(1 / n, 1 / n, \ldots, 1 / n)^{T}$, then GIVDHFUUBLHA and GIVDHFUUBLHG operators reduce to the GIVDHFUUBLWA and GIVDHFUUBLWG operators, respectively;

(2) If $\omega=(1 / n, 1 / n, \ldots, 1 / n)^{T}$, then GIVDHFUUBLHA and GIVDHFUUBLHG operators reduce to the GIVDHFUUBLOWA and GIVDHFUUBLOWG operators, respectively.

\section{(2) If $\lambda=1$}

(1) If $w=(1 / n, 1 / n, \ldots, 1 / n)^{T}$, then GIVDHFUUBLHA and GIVDHFUUBLHG operators reduce to the IVDHF_UUBL weighted average (IVDHFUUBLWA) and IVDHF_UUBL weighted geometric (IVDHFUUBLWG) operators, respectively, where 


$$
\begin{aligned}
& \operatorname{IVDHFUUBLWA} A_{\omega}\left(s d_{1}, s d_{2}, \ldots, s d_{n}\right)=\bigoplus_{j=1}^{n} \omega_{j} s d_{j} \\
& =\bigcup_{\left(\widetilde{s}_{q_{j}}, \widetilde{h}_{j}, \widetilde{g}_{j}\right) \in s d_{j}}\left(\left[s_{\sum_{j=1}^{n} \omega_{j}\left(\Delta_{t_{0}}^{-1}\left(T F_{t_{0}}^{t_{j_{1}}}\left(\psi\left(s_{\alpha_{j}}\right)\right)\right)\right.}, s_{\sum_{j=1}^{n} \omega_{j}\left(\Delta_{t_{0}}^{-1}\left(T F_{t_{0}}^{t_{j_{2}}}\left(\psi\left(s_{\beta_{j}}\right)\right)\right)\right.}\right],\right. \\
& \left.\bigcup_{\left[\mu_{j}^{L}, \mu_{j}^{U}\right] \in \widetilde{h}_{j},\left[\nu_{j}^{L}, \nu_{j}^{U}\right] \in \widetilde{g}_{j}}\left\{\left\{\left[1-\prod_{j=1}^{n}\left(1-\mu_{j}^{L}\right)^{\omega_{j}}, 1-\prod_{j=1}^{n}\left(1-\mu_{j}^{U}\right)^{\omega_{j}}\right]\right\},\left\{\left[\prod_{j=1}^{n}\left(v_{j}^{L}\right)^{\omega_{j}}, \prod_{j=1}^{n}\left(v_{j}^{U}\right)^{\omega_{j}}\right]\right\}\right\}\right) \\
& \operatorname{IVDHFUUBLWG} G_{\omega}\left(s d_{1}, s d_{2}, \ldots, s d_{n}\right)=\bigotimes_{j=1}^{n} s d_{j}{ }^{{ }}{ }_{j}
\end{aligned}
$$

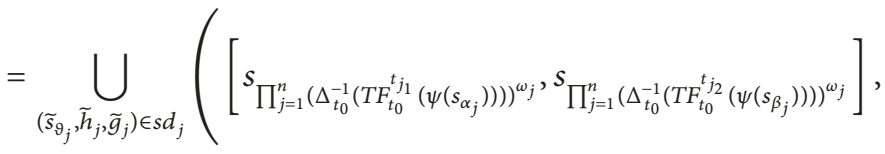

$$
\begin{aligned}
& \left.\bigcup_{\left.\left[\mu_{j}^{L}, \mu_{j}^{U}\right] \in \widetilde{h}_{j}, \nu_{j}^{L}, v_{j}^{U}\right] \in \tilde{g}_{j}}\left\{\left\{\left[\prod_{j=1}^{n}\left(\mu_{j}^{L}\right)^{\omega_{j}}, \prod_{j=1}^{n}\left(\mu_{j}^{U}\right)^{\omega_{j}}\right]\right\},\left\{\left[1-\prod_{j=1}^{n}\left(1-v_{j}^{L}\right)^{\omega_{j}}, 1-\prod_{j=1}^{n}\left(1-v_{j}^{U}\right)^{\omega_{j}}\right]\right\}\right\}\right)
\end{aligned}
$$

(2) If $\omega=(1 / n, 1 / n, \ldots, 1 / n)^{T}$, then GIVDHFUUBLHA and GIVDHFUUBLHG operators reduce to the IVDHF_UUBL ordered weighted average (IVDHFUUBLOWA) and IVDHF_ UUBL ordered weighted geometric (IVDHFUUBLOWG) operators, respectively, where

$$
\begin{aligned}
& \operatorname{IVDHFUUBLOWA}_{w}\left(s d_{1}, s d_{2}, \ldots, s d_{n}\right)=\bigoplus_{j=1}^{n} w_{j} s d_{\sigma(j)} \\
& =\bigcup_{\left.\left(\widetilde{s}_{\sigma_{\sigma(j)}} \widetilde{h}_{\sigma(j)}\right) \widetilde{g}_{\sigma(j)}\right) \in s d_{\sigma(j)}}\left(\left[s_{\sum_{j=1}^{n} w_{j} \Delta_{t_{0}}^{-1}\left(T F_{t_{0}}^{t_{j}}\left(\psi\left(s_{\alpha_{\sigma(j)}}\right)\right)\right)}, s_{\sum_{j=1}^{n} w_{j} \Delta_{t_{0}}^{-1}\left(T F_{t_{0}}^{t_{j}}\left(\psi\left(s_{\beta_{\sigma(j)}}\right)\right)\right)}\right],\right. \\
& \left.\left\{\bigcup_{\left[\mu_{\sigma(j)}^{L}, \mu_{\sigma(j)}^{U}\right] \in \tilde{h}_{\sigma(j)},\left[\nu_{\sigma(j)}^{L}, \nu_{\sigma(j)}^{U}\right] \in \tilde{g}_{\sigma(j)}}\left\{\left\{\left[1-\prod_{j=1}^{n}\left(1-\mu_{\sigma(j)}^{L}\right)\right)^{w_{j}}, 1-\prod_{j=1}^{n}\left(1-\mu_{\sigma(j)}^{U}\right)^{w_{j}}\right]\right\},\left\{\left[\prod_{j=1}^{n}\left(\nu_{\sigma(j)}^{L}\right)^{w_{j}}, \prod_{j=1}^{n}\left(v_{\sigma(j)}^{U}\right){ }^{w_{j}}\right]\right\}\right\}\right) ; \\
& \operatorname{IVDHFUUBLOWG} G_{w}\left(s d_{1}, s d_{2}, \ldots, s d_{n}\right)=\bigotimes_{j=1}^{n} s d_{\sigma(j)} w_{j}
\end{aligned}
$$

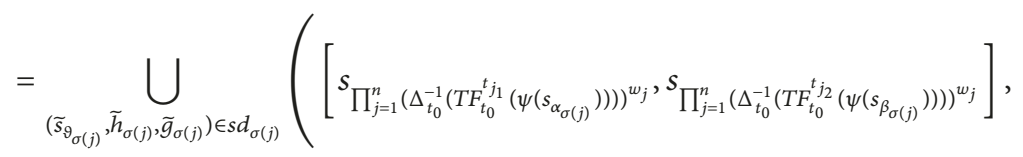

$$
\begin{aligned}
& \left\{\bigcup_{\left[\mu_{\sigma(j)}^{L}, \mu_{\sigma(j)}^{U}\right] \in \tilde{h}_{\sigma(j)},\left[\nu_{\sigma(j)}^{L}, \nu_{\sigma(j)}^{U}\right] \in \tilde{g}_{\sigma(j)}}\left\{\left\{\left[\prod_{j=1}^{n}\left(\mu_{\sigma(j)}^{L}\right)^{w_{j}}, \prod_{j=1}^{n}\left(\mu_{\sigma(j)}^{U}\right)^{w_{j}}\right]\right\},\left\{\left[1-\prod_{j=1}^{n}\left(1-\nu_{\sigma(j)}^{L}\right)^{w_{j}}, 1-\prod_{j=1}^{n}\left(1-v_{\sigma(j)}^{U}\right)^{w_{j}}\right]\right\}\right\} .\right.
\end{aligned}
$$


Overall, we can conclude the relationship between all these proposed aggregation operators as shown in Theorem 14.
Utilize the programming model (M-1) in Section 4.1; by calculating (29), we get

\section{Computation Steps of Algorithm I}

Step 1. Determine argument-dependent weighting vector $\omega^{A D}$.

$$
\omega^{A D}=(0.1383,0.171,0.1597,0.1059,0.102,0.0902,0.1202,0.1127)^{T} .
$$

Step 2. Determine attributes interdependency-based weighting vector $\omega^{A I}$.

After processing Step 4 in Algorithm II, the influencing and influenced degrees of each attribute can be obtained as shown in Table 2. Then according to (33) and (34), weighting vector based on attributes interdependency is determined as

$$
\omega^{A I}=(0.1248,0.1149,0.1158,0.121,0.1379,0.1255,0.135,0.1251)^{T} .
$$

Step 3. Obtain synthesized attributes weighting vector.

To comprehensively consider attribute weighting information, synthesized weighting vector is computed by (19) in which $\alpha$ and $\beta$ are set as 0.5 ,

$$
\begin{aligned}
& \omega \\
& =(0.1316,0.143,0.1377,0.1135,0.12,0.1078,0.1276,0.1188)^{T} .
\end{aligned}
$$

Step 4. Check order inducing requirements.

After consulting the expert team, if there is no group attitudinal characters (i.e., order inducing vectors), then go to Step 5; otherwise go to Step 6.

Step 5. Derive overall IVDHF_UUBLNs $\tilde{r}_{j}$ of each alternative $x_{j}(j=1,2,3)$.

Here, we choose GIVDHFUUBLHA operator in Definition 12 and assign $\lambda=2$. The associated position weighting vector is assigned as $w=(0,0,0.15,0.25,0.25,0.25,0.1,0)^{T}$, which is acquired by commonly used fuzzy semantic quantitative operator with parameters $(a, b)$ set as $(0.3,0.8)$ [99]. Then we get the values of $\widetilde{r}_{1}, \widetilde{r}_{2}$, and $\widetilde{r}_{3}$. Taking $\widetilde{r}_{3}$, for example, we have

$$
\begin{gathered}
\widetilde{r}_{3}=\left(\left[s_{3.5754}, s_{4.8345}\right],\{\{[0.36594,0.48336],\right. \\
{[0.36594,0.48336],[0.39856,0.51409],} \\
{[0.39856,0.51409],[0.36594,0.48336],} \\
{[0.36594,0.48336],[0.39856,0.51409],} \\
[0.39856,0.51409]\},\{[0.29622,0.39832], \\
{[0.30123,0.4036],[0.29622,0.39832],} \\
[0.30123,0.4036]\}\}) .
\end{gathered}
$$

Later on, $\widetilde{r}_{1}, \widetilde{r}_{2}$, and $\widetilde{r}_{3}$ will be fed into Step 8 to calculate scores and accuracy degrees of each contractor.

Step 6. Derive order-inducing vectors $\varepsilon$.

According to the TOPSIS-based method described in Algorithm III, decision-makers are required to put forward both IVDHF_UUBL ideal alternatives and IVDHF_UUBL negative ideal alternatives. As listed in Table 3, the ideal solution and negative ideal solution are collected, based on which the order-inducing vectors $\varepsilon$ are derived by sorting the coefficients generated out of (37).

Step 7. Utilize induced generalized aggregation operators to get the overall IVDHFUBBLNs $\tilde{r}_{j}(j=1,2, \ldots, m)$ of each alternative $x_{j}$.

After order-inducing vector $\varepsilon$ is obtained, IGIVDHFUUBLHA operator defined in Definition 13 is chosen for information aggregation, $\lambda$ and position weights are set same as in Step 5, and then we can obtain $\widetilde{r}_{1}, \widetilde{r}_{2}$ and $\tilde{r}_{3}$. Taking $\widetilde{r}_{3}$ as an example, we have

$$
\begin{aligned}
\widetilde{r}_{3}= & \left(\left[s_{4.0762}, s_{5.877}\right],\{\{[0.31061,0.43001],\right. \\
& {[0.31061,0.43548],[0.34999,0.46638], } \\
& {[0.34999,0.47123],[0.31061,0.43001], } \\
& {[0.31061,0.43548],[0.34999,0.46638], } \\
& {[0.34999,0.47123]\},\{[0.39075,0.49203],} \\
& {[0.39075,0.49203],[0.40918,0.51064], } \\
& {[0.40918,0.51064]\}\}) ; }
\end{aligned}
$$

Step 8. Calculate scores and accuracy degrees of each solution. 
Now we can utilize the score function and accuracy function defined in Definition 4 to calculate scores $S\left(\widetilde{r}_{j}\right)$ and accuracy degrees $P\left(\widetilde{r}_{j}\right)$ of each $\widetilde{r}_{j}$. For each $\widetilde{r}_{j}$ generated from Step 5, we have $S_{\text {GIVDHFUUBLHA }}\left(\widetilde{r}_{1}\right)=-0.146$, $S_{\text {GIVDHFUUBLHA }}\left(\widetilde{r}_{2}\right)=2.157$, and $S_{\text {GIVDHFUUBLHA }}\left(\widetilde{r}_{3}\right)=$ 0.381 . And for each $\widetilde{r}_{j}$ generated from Step 7, we have $S_{\text {I-GIVDHFUUBLHA }}\left(\widetilde{r}_{1}\right)=0.136, S_{\text {I-GIVDHFUUBLHA }}\left(\widetilde{r}_{2}\right)=1.962$, and $S_{I-G I V D H F U U B L H A}\left(\widetilde{r}_{3}\right)=-0.299$.

Step 9. Generate final ranking results.

According to the scores obtained from Step 5 and the comparative rules defined in Definition 5, the ranking order is $x_{2}>x_{3}>x_{1}$, indicating that $x_{2}$ is the most appropriate solution. Regarding the scores obtained from Step 7, the ranking order is $x_{2}>x_{1}>x_{3}$, indicating that $x_{2}$ is still the most appropriate solution and solution $x_{1}$ is better than solution $x_{3}$. Ranking results are then collected in Table 4 for clarity.

\section{Data Availability}

The data used to support the findings of this study are included within the article.

\section{Conflicts of Interest}

The authors declare that there are no conflicts of interest regarding the publication of this paper.

\section{Acknowledgments}

The authors would like to greatly thank joint-support by the National Natural Science Foundation of China (nos. 71701181, 71771075, and 71331002), the Social Science Foundation of Ministry of Education of China (no. 16YJC630094), and the Natural Science Foundation of Zhejiang Province of China (LQ17G010002 and LY18G010010).

\section{References}

[1] V. W. Y. Tam and C. M. Tam, "Waste reduction through incentives: A case study," Building Research and Information, vol. 36, no. 1, pp. 37-43, 2008.

[2] Z. Majdalani, M. Ajam, and T. Mezher, "Sustainability in the construction industry: A Lebanese case study," Construction Innovation, vol. 6, no. 1, pp. 33-46, 2006.

[3] V. W. Y. Tam, L. Y. Shen, I. W. H. Fung, and J. Y. Wang, "Controlling construction waste by implementing governmental ordinances in Hong Kong," Construction Innovation, vol. 7, no. 2, pp. 149-166, 2007.

[4] P. O. Akadiri, P. O. Olomolaiye, and E. A. Chinyio, "Multicriteria evaluation model for the selection of sustainable materials for building projects," Automation in Construction, vol. 30, pp. 113-125, 2013.

[5] M. Safa, A. Shahi, C. T. Haas et al., "Competitive intelligence (CI) for evaluation of construction contractors," Automation in Construction, vol. 59, article no. 1862, pp. 149-157, 2015.
[6] G. Y. Qi, L. Y. Shen, S. X. Zeng, and O. J. Jorge, “The drivers for contractors' green innovation: An industry perspective," Journal of Cleaner Production, vol. 18, no. 14, pp. 1358-1365, 2010.

[7] A. Rostami and C. F. Oduoza, "Key risks in construction projects in Italy: Contractors' perspective," Engineering, Construction and Architectural Management, vol. 24, no. 3, pp. 451462, 2017.

[8] R. Liang, Z. Sheng, and X. Wang, "Methods Dealing with Complexity in Selecting Joint Venture Contractors for LargeScale Infrastructure Projects," Complexity, vol. 2018, Article ID 8705134, 14 pages, 2018.

[9] M. Safa, C. T. Haas, K. W. Hipel, and J. Gray, "Front End Planning Tool (FEPT) Based on an Electronic Process Management," Journal of Construction Engineering and Project Management, vol. 3, no. 2, pp. 1-12, 2013.

[10] A. Nieto-Morote and F. Ruz-Vila, "A fuzzy multi-criteria decision-making model for construction contractor prequalification," Automation in Construction, vol. 25, pp. 8-19, 2012.

[11] X. Qi, C. Liang, and J. Zhang, "Generalized cross-entropy based group decision making with unknown expert and attribute weights under interval-valued intuitionistic fuzzy environment," Computers \& Industrial Engineering, vol. 79, pp. 52-64, 2015.

[12] H. Zimmermann, Fuzzy Set Theory-and Its Applications, Kluwer Academic, Boston, Mass, USA, 2001.

[13] L. A. Zadeh, "The concept of a linguistic variable and its application to approximate reasoning I," Information Sciences, vol. 8, pp. 199-249, 1975.

[14] Z. Xu, "A method for multiple attribute decision making with incomplete weight information in linguistic setting," Knowledge-Based Systems, vol. 20, no. 8, pp. 719-725, 2007.

[15] Y. Ju, A. Wang, and X. Liu, "Evaluating emergency response capacity by fuzzy AHP and 2-tuple fuzzy linguistic approach," Expert Systems with Applications, vol. 39, no. 8, pp. 6972-6981, 2012.

[16] B. T. Nuong, K.-W. Kim, L. Prathumratana et al., "Sustainable development in the mining sector and its evaluation using fuzzy AHP (Analytic Hierarchy Process) approach," Geosystem Engineering, vol. 14, no. 1, pp. 43-50, 2011.

[17] S. Kusi-Sarpong, C. Bai, J. Sarkis, and X. Wang, "Green supply chain practices evaluation in the mining industry using a joint rough sets and fuzzy TOPSIS methodology," Resources Policy, vol. 46, pp. 86-100, 2015.

[18] E. K. Zavadskas, Z. Turskis, and J. Antucheviciene, "Selecting a contractor by using a novel method for multiple attribute analysis: Weighted aggregated sum product assessment with grey values (WASPAS-G)," Studies in Informatics and Control, vol. 24, no. 2, pp. 141-150, 2015.

[19] B. Vahdani, S. M. Mousavi, H. Hashemi, M. Mousakhani, and R. Tavakkoli-Moghaddam, "A new compromise solution method for fuzzy group decision-making problems with an application to the contractor selection," Engineering Applications of Artificial Intelligence, vol. 26, no. 2, pp. 779-788, 2013.

[20] H. M. Alhumaidi, "Construction contractors ranking method using multiple decision-makers and multiattribute fuzzy weighted average," Journal of Construction Engineering and Management, vol. 141, no. 4, 2015.

[21] V. Torra, "Hesitant fuzzy sets," International Journal of Intelligent Systems, vol. 25, no. 6, pp. 529-539, 2010.

[22] Z. Xu, Hesitant Fuzzy Sets Theory, vol. 314 of Studies in Fuzziness and Soft Computing, Springer, Berlin, Germany, 2014. 
[23] M. P. Borujeni and H. Gitinavard, "Evaluating the sustainable mining contractor selection problems: An imprecise last aggregation preference selection index method," Journal of Sustainable Mining, vol. 16, no. 4, pp. 207-218, 2017.

[24] P. S.-W. Fong and S. K.-Y. Choi, "Final contractor selection using the analytical hierarchy process," Construction Management and Economics, vol. 18, no. 5, pp. 547-557, 2000.

[25] M. Darvish, M. Yasaei, and A. Saeedi, "Application of the graph theory and matrix methods to contractor ranking," International Journal of Project Management, vol. 27, no. 6, pp. 610-619, 2009.

[26] Z. Hatush and M. Skitmore, "Contractor Selection Using Multicriteria Utility Theory: An Additive Model," Building and Environment, vol. 33, no. 2-3, pp. 105-115, 1998.

[27] B.-G. Hwang, X. Zhao, and G. S. Yu, "Risk identification and allocation in underground rail construction joint ventures: contractors' perspective," Journal of Civil Engineering and Management, vol. 22, no. 6, pp. 758-767, 2016.

[28] L. Y. Shen, W. Lu, Q. Shen, and H. Li, "A computer-aided decision support system for assessing a contractor's competitiveness," Automation in Construction, vol. 12, no. 5, pp. 577-587, 2003.

[29] B. G. Hwang and H. B. Ng, "Project network management: risks and contributors from the viewpoint of contractors and sub-contractors," Technological and Economic Development of Economy, vol. 22, no. 4, pp. 631-648, 2016.

[30] A. M. Anvuur and M. M. Kumaraswamy, "Conceptual model of partnering and alliancing," Journal of Construction Engineering and Management, vol. 133, no. 3, pp. 225-234, 2007.

[31] A. H. I. Lee, H. Kang, C. F. Hsu, and H. Hung, "A green supplier selection model for high-tech industry," Expert Systems with Applications, vol. 36, no. 4, pp. 7917-7927, 2009.

[32] C. Y. Chiou, C. W. Hsu, and W. Y. Hwang, "Comparative investigation on green supplier selection of the American, Japanese and Taiwanese Electronics Industry in China," in Proceedings of the 2008 IEEE International Conference on Industrial Engineering and Engineering Management, IEEM 2008, pp. 1909-1914, Singapore, December 2008.

[33] P. Humphreys, R. McIvor, and F. Chan, "Using case-based reasoning to evaluate supplier environmental management performance," Expert Systems with Applications, vol. 25, no. 2, pp. 141-153, 2003.

[34] P. Humphreys, A. McCloskey, R. McIvor, L. Maguire, and C. Glackin, "Employing dynamic fuzzy membership functions to assess environmental performance in the supplier selection process," International Journal of Production Research, vol. 44, no. 12, pp. 2379-2419, 2006.

[35] R. J. Lin, "Using fuzzy DEMATEL to evaluate the green supply chain management practices," Journal of Cleaner Production, vol. 40, pp. 32-39, 2013.

[36] Y. Yang and L. Wu, "Extension Method for Green Supplier Selection," in Proceedings of the 2008 4th International Conference on Wireless Communications, Networking and Mobile Computing (WiCOM), pp. 1-4, Dalian, China, October 2008.

[37] B. Liu and H.-J. Liu, "A research on supplier assessment indices system of green purchasing," in Proceedings of the International Conference on Measuring Technology and Mechatronics Automation, ICMTMA 2010, pp. 314-317, China, March 2010.

[38] R. Lin, X. Zhao, H. Wang, and G. Wei, "Hesitant fuzzy linguistic aggregation operators and their application to multiple attribute decision making," Journal of Intelligent \& Fuzzy Systems: Applications in Engineering and Technology, vol. 27, no. 1, pp. 49-63, 2014.

[39] G. Wei, "Interval-valued dual hesitant fuzzy uncertain linguistic aggregation operators in multiple attribute decision making," Journal of Intelligent \& Fuzzy Systems: Applications in Engineering and Technology, vol. 33, no. 3, pp. 1881-1893, 2017.

[40] A. Mardani, A. Jusoh, and E. K. Zavadskas, "Fuzzy multiple criteria decision-making techniques and applications-two decades review from 1994 to 2014," Expert Systems with Applications, vol. 42, no. 8, pp. 4126-4148, 2015.

[41] F. Meng, X. Chen, and Q. Zhang, "Generalized hesitant fuzzy generalized Shapley-Choquet integral operators and their application in decision making," International Journal of Fuzzy Systems, vol. 16, no. 3, pp. 400-410, 2014.

[42] S. Cevik Onar, B. Oztaysi, and C. Kahraman, "Strategic Decision Selection Using Hesitant fuzzy TOPSIS and Interval Type-2 Fuzzy AHP: A case study," International Journal of Computational Intelligence Systems, vol. 7, no. 5, pp. 1002-1021, 2014.

[43] C. Tan, W. Yi, and X. Chen, "Hesitant fuzzy Hamacher aggregation operators for multicriteria decision making," Applied Soft Computing, vol. 26, pp. 325-349, 2015.

[44] X. Zhang and Z. Xu, "Hesitant fuzzy QUALIFLEX approach with a signed distance-based comparison method for multiple criteria decision analysis," Expert Systems with Applications, vol. 42, no. 2, pp. 873-884, 2015.

[45] Bin Zhu, Zeshui Xu, and Meimei Xia, "Dual Hesitant Fuzzy Sets," Journal of Applied Mathematics, vol. 2012, Article ID 879629, 13 pages, 2012.

[46] B. Zhu and $\mathrm{Z}$. Xu, "Some results for dual hesitant fuzzy sets," Journal of Intelligent \& Fuzzy Systems. Applications in Engineering and Technology, vol. 26, no. 4, pp. 1657-1668, 2014.

[47] Y. Ju, X. Liu, and S. Yang, "Interval-valued dual hesitant fuzzy aggregation operators and their applications to multiple attribute decision making," Journal of Intelligent \& Fuzzy Systems: Applications in Engineering and Technology, vol. 27, no. 3, pp. 1203-1218, 2014.

[48] W. Zhang, X. Li, and Y. Ju, "Some Aggregation Operators Based on Einstein Operations under Interval-Valued Dual Hesitant Fuzzy Setting and Their Application," Mathematical Problems in Engineering, vol. 2014, Article ID 958927, 21 pages, 2014.

[49] B. Farhadinia, "Study on division and subtraction operations for hesitant fuzzy sets, interval-valued hesitant fuzzy sets and typical dual hesitant fuzzy sets," Journal of Intelligent \& Fuzzy Systems: Applications in Engineering and Technology, vol. 28, no. 3, pp. 1393-1402, 2015.

[50] S. Yang and Y. Ju, "A GRA method for investment alternative selection under dual hesitant fuzzy environment with incomplete weight information," Journal of Intelligent \& Fuzzy Systems: Applications in Engineering and Technology, vol. 28, no. 4, pp. 1533-1543, 2015.

[51] R. M. Rodriguez, L. Martinez, and F. Herrera, "Hesitant fuzzy linguistic term sets for decision making," IEEE Transactions on Fuzzy Systems, vol. 20, no. 1, pp. 109-119, 2012.

[52] Z. S. Xu, "Uncertain linguistic aggregation operators based approach to multiple attribute group decision making under uncertain linguistic environment," Information Sciences, vol. 168, no. 1-4, pp. 171-184, 2004.

[53] J.-Q. Wang, J.-T. Wu, H.-Y. Zhang, and X.-H. Chen, "Intervalvalued hesitant fuzzy linguistic sets and their applications in multi-criteria decision-making problems," Information Sciences, vol. 288, pp. 55-72, 2014. 
[54] S. Yang and Y. Ju, "Dual hesitant fuzzy linguistic aggregation operators and their applications to multi-attribute decision making," Journal of Intelligent \& Fuzzy Systems: Applications in Engineering and Technology, vol. 27, no. 4, pp. 1935-1947, 2014.

[55] Z. Tao, X. Liu, H. Chen, and L. Zhou, "Using new version of extended t-norms and s-norms for aggregating interval linguistic labels," IEEE Transactions on Systems Man Cybernetics Systems, vol. vol, p. 15, 2016.

[56] L. Martínez and F. Herrera, "An overview on the 2-tuple linguistic model for computing with words in decision making: extensions, applications and challenges," Information Sciences, vol. 207, pp. 1-18, 2012.

[57] E. Herrera-Viedma and A. G. López-Herrera, "A model of an information retrieval system with unbalanced fuzzy linguistic information," International Journal of Intelligent Systems, vol. 22, no. 11, pp. 1197-1214, 2007.

[58] L. Martínez, M. Espinilla, J. Liu, L. G. Pérez, and P. J. Sánchez, "An evaluation model with unbalanced linguistic information applied to olive oil sensory evaluation," Journal of MultipleValued Logic and Soft Computing, vol. 15, no. 2-3, pp. 229-251, 2009.

[59] F. Herrera, E. Herrera-Viedma, and L. Martínez, "A fuzzy linguistic methodology to deal with unbalanced linguistic term sets," IEEE Transactions on Fuzzy Systems, vol. 16, no. 2, pp. 354$370,2008$.

[60] D. Joshi and S. Kumar, "Interval-valued intuitionistic hesitant fuzzy Choquet integral based TOPSIS method for multi-criteria group decision making," European Journal of Operational Research, vol. 248, no. 1, pp. 183-191, 2016.

[61] J. M. Merigó and M. Casanovas, "Decision-making with distance measures and induced aggregation operators," Computers \& Industrial Engineering, vol. 60, no. 1, pp. 66-76, 2011.

[62] L. Marin, A. Valls, D. Isern, A. Moreno, and J. M. Merigó, "Induced unbalanced linguistic ordered weighted average and its application in multiperson decision making," The Scientific World Journal, vol. 2014, 2014.

[63] A. Baykasoğlu, V. Kaplanoglu, Z. D. U. Durmuşoglu, and C. Şahin, "Integrating fuzzy DEMATEL and fuzzy hierarchical TOPSIS methods for truck selection," Expert Systems with Applications, vol. 40, no. 3, pp. 899-907, 2013.

[64] S. K. Patil and R. Kant, "A hybrid approach based on fuzzy DEMATEL and FMCDM to predict success of knowledge management adoption in supply chain," Applied Soft Computing, vol. 18, pp. 126-135, 2014.

[65] S. Liyin and Y. Hong, "Improving environmental performance by means of empowerment of contractors," Management of Environmental Quality: An International Journal, vol. 17, no. 3, pp. 242-257, 2006.

[66] Z. Wu, A. T. W. Yu, and L. Shen, "Investigating the determinants of contractor's construction and demolition waste management behavior in Mainland China," Waste Management, vol. 60, pp. 290-300, 2017.

[67] E. K. Zavadskas, T. Vilutiene, Z. Turskis, and J. Tamosaitiene, "Contractor selection for construction works by applying saw-g and topsis grey techniques," Journal of Business Economics and Management, vol. 11, no. 1, pp. 34-55, 2010.

[68] Y. Tan, L. Shen, and H. Yao, "Sustainable construction practice and contractors' competitiveness: A preliminary study," Habitat International, vol. 35, no. 2, pp. 225-230, 2011.

[69] Y. Kazancoglu, I. Kazancoglu, and M. Sagnak, "A new holistic conceptual framework for green supply chain management performance assessment based on circular economy," Journal of Cleaner Production, vol. 195, pp. 1282-1299, 2018.

[70] A. Nureize and J. Watada, "Multi-attribute decision making in contractor selection under hybrid uncertainty," Journal of Advanced Computational Intelligence and Intelligent Informatics, vol. 15, no. 4, pp. 465-472, 2011.

[71] J. Sarkis, L. M. Meade, and A. R. Presley, "Incorporating sustainability into contractor evaluation and team formation in the built environment," Journal of Cleaner Production, vol. 31, pp. 40-53, 2012.

[72] N. Romeli, F. M. Halil, F. Ismail, and A. S. Shukor, "Economic Challenges in Joint Venture Infrastructure Projects: Towards Contractor's Quality of Life," Procedia - Social and Behavioral Sciences, vol. 234, pp. 19-27, 2016.

[73] R. K. Mavi, M. Goh, and N. Zarbakhshnia, "Sustainable thirdparty reverse logistic provider selection with fuzzy SWARA and fuzzy MOORA in plastic industry," The International Journal of Advanced Manufacturing Technology, vol. 91, no. 5-8, pp. 24012418, 2017.

[74] Z.-Y. Zhao, C. Tang, X. Zhang, and M. Skitmore, "Agglomeration and Competitive Position of Contractors in the International Construction Sector," Journal of Construction Engineering and Management, vol. 143, no. 6, 2017.

[75] E. U. Olugu, K. Y. Wong, and A. M. Shaharoun, "Development of key performance measures for the automobile green supply chain," Resources, Conservation \& Recycling, vol. 55, no. 6, pp. 567-579, 2011.

[76] Q. Zhu and J. Sarkis, "An inter-sectoral comparison of green supply chain management in China: drivers and practices," Journal of Cleaner Production, vol. 14, no. 5, pp. 472-486, 2006.

[77] K. Govindan, S. Rajendran, J. Sarkis, and P. Murugesan, "Multi criteria decision making approaches for green supplier evaluation and selection: a literature review," Journal of Cleaner Production, vol. 98, pp. 66-83, 2015.

[78] O. Feyziog̃lu and G. Büyüközkan, "Evaluation of Green Suppliers Considering Decision Criteria Dependencies," in Multiple Criteria Decision Making for Sustainable Energy and Transportation Systems, vol. 634 of Lecture Notes in Economics and Mathematical Systems, pp. 145-154, Springer Berlin Heidelberg, Berlin, Heidelberg, 2010.

[79] P. D. Rwelamila, A. A. Talukhaba, and A. B. Ngowi, "Project procurement systems in the attainment of sustainable construction," Sustainable Development, vol. 8, no. 1, pp. 39-50, 2000.

[80] H. Fergusson and D. A. Langford, "Strategies for managing environmental issues in construction organizations," Engineering, Construction and Architectural Management, vol. 13, no. 2, pp. 171-185, 2006.

[81] S. Sharma and H. Vredenburg, "Proactive corporate environmental strategy and the development of competitively valuable organizational capabilities," Strategic Management Journal, vol. 19, no. 8, pp. 729-753, 1998.

[82] C. M. Tam, V. W. Y. Tam, L. Y. Tam, and D. Drew, "Mapping approach for examining waste management on construction sites," Journal of Construction Engineering and Management, vol. 130, no. 4, pp. 472-481, 2004.

[83] P. Burillo and H. Bustince, "Entropy on intuitionistic fuzzy sets and on interval-valued fuzzy sets," Fuzzy Sets and Systems, vol. 78, no. 3, pp. 305-316, 1996.

[84] C. Q. Tan and X. H. Chen, "Intuitionistic fuzzy Choquet integral operator for multi-criteria decision making," Expert Systems with Applications, vol. 37, no. 1, pp. 149-157, 2010. 
[85] P. P. Wakker, Additive representations of preferences, Theory and Decision Library. Series C: Game Theory, Mathematical Programming and Operations Research, Kluwer Academic Publishers Group, Dordrecht, 1989.

[86] Z. S. Xu and M. Xia, "Distance and similarity measures for hesitant fuzzy sets," Information Sciences, vol. 181, no. 11, pp. 2128-2138, 2011.

[87] R. R. Yager, “Generalized OWA aggregation operators," Fuzzy Optimization and Decision Making, vol. 3, no. 1, pp. 93-107, 2004.

[88] R. R. Yager and D. P. Filev, "Induced ordered weighted averaging operators," IEEE Transactions on Systems, Man, and Cybernetics, Part B: Cybernetics, vol. 29, no. 2, pp. 141-150, 1999.

[89] J. M. Merigó and M. Casanovas, "Induced aggregation operators in the Euclidean distance and its application in financial decision making," Expert Systems with Applications, vol. 38, no. 6, pp. 7603-7608, 2011.

[90] Z. Xu and M. Xia, "Induced generalized intuitionistic fuzzy operators," Knowledge-Based Systems, vol. 24, no. 2, pp. 197-209, 2011.

[91] Y. M. Wang, "Using the method of maximizing deviations to make decision for multi-indices," Journal of Systems Engineering and Electronics, vol. 8, pp. 21-26, 1997.

[92] C. T. Chen, P. F. Pai, and W. Z. Hung, "An integrated methodology using linguistic PROMETHEE and maximum deviation method for third-party logistics supplier selection," International Journal of Computational Intelligence Systems, vol. 3, no. 4, pp. 438-451, 2010.

[93] Z. S. Xu, "Approaches to multiple attribute group decision making based on intuitionistic fuzzy power aggregation operators," Knowledge-Based Systems, vol. 24, no. 6, pp. 749-760, 2011.

[94] Z. S. Xu, "Choquet integrals of weighted intuitionistic fuzzy information," Information Sciences, vol. 180, no. 5, pp. 726-736, 2010.

[95] Y. Ju, S. Yang, and X. Liu, "Some new dual hesitant fuzzy aggregation operators based on Choquet integral and their applications to multiple attribute decision making," Journal of Intelligent \& Fuzzy Systems: Applications in Engineering and Technology, vol. 27, no. 6, pp. 2857-2868, 2014.

[96] D. Dalalah, M. Hayajneh, and F. Batieha, "A fuzzy multi-criteria decision making model for supplier selection," Expert Systems with Applications, vol. 38, no. 7, pp. 8384-8391, 2011.

[97] H. Liu, J. X. You, Ch. Lu, and Y. Z. Chen, "Evaluating health-care waste treatment technologies using a hybrid multi-criteria decision making model," Renewable \& Sustainable Energy Reviews, vol. 41, pp. 932-942, 2015.

[98] F. Chiclana, E. Herrera-Viedma, F. Herrera, and S. Alonso, "Some induced ordered weighted averaging operators and their use for solving group decision-making problems based on fuzzy preference relations," European Journal of Operational Research, vol. 182, no. 1, pp. 383-399, 2007.

[99] L. A. Zadeh, "A computational approach to fuzzy quantifiers in natural languages," Computers \& Mathematics with Applications, vol. 9, no. 1, pp. 149-184, 1983.

[100] Z. Zhang, "Generalized Atanassov's intuitionistic fuzzy power geometric operators and their application to multiple attribute group decision making," Information Fusion, vol. 14, no. 4, pp. 460-486, 2013.

[101] Z. Zhang, C. Wang, D. Tian, and K. Li, "Induced generalized hesitant fuzzy operators and their application to multiple attribute group decision making," Computers \& Industrial Engineering, vol. 67, no. 1, pp. 116-138, 2014.
[102] R. R. Yager, "Induced aggregation operators," Fuzzy Sets and Systems, vol. 137, no. 1, pp. 59-69, 2003.

[103] J. M. Merigó and M. Casanovas, "Induced and heavy aggregation operators with distance measures," Journal of Systems Engineering and Electronics, vol. 21, no. 3, pp. 431-439, 2010.

[104] G. Wei, "Some induced geometric aggregation operators with intuitionistic fuzzy information and their application to group decision making," Applied Soft Computing, vol. 10, no. 2, pp. 423-431, 2010.

[105] Jun-Ling Zhang and Xiao-Wen Qi, “Induced Interval-Valued Intuitionistic Fuzzy Hybrid Aggregation Operators with TOPSIS Order-Inducing Variables," Journal of Applied Mathematics, vol. 2012, Article ID 245732, 24 pages, 2012.

[106] J. M. Merigó, A. M. Gil-Lafuente, L.-G. Zhou, and H.-Y. Chen, "Induced and linguistic generalized aggregation operators and their application in linguistic group decision making," Group Decision and Negotiation, vol. 21, no. 4, pp. 531-549, 2012.

[107] Y. Dong, C.-C. Li, and F. Herrera, "Connecting the numerical scale model to the unbalanced linguistic term sets," in Proceedings of the 2014 IEEE International Conference on Fuzzy Systems, FUZZ-IEEE 2014, pp. 455-462, China, July 2014. 


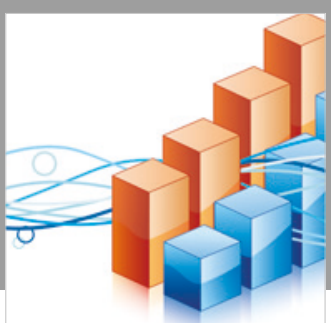

Advances in

Operations Research

\section{-n-m}
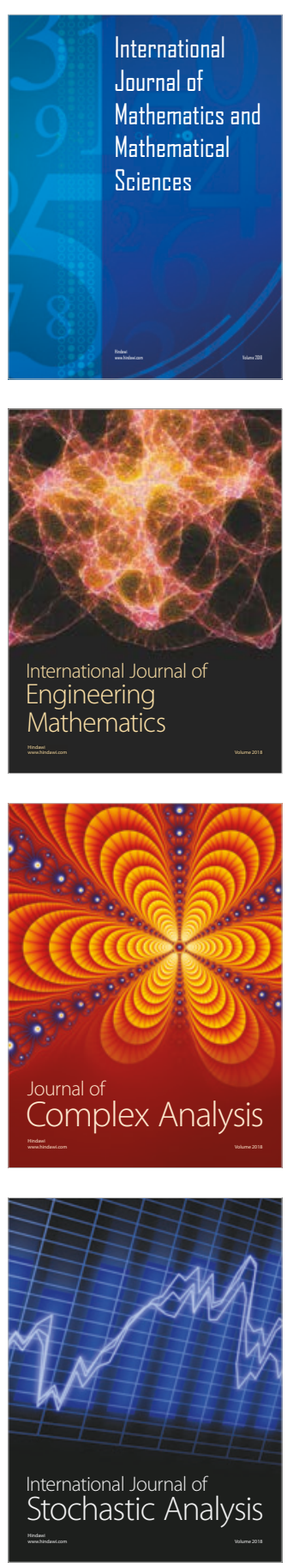
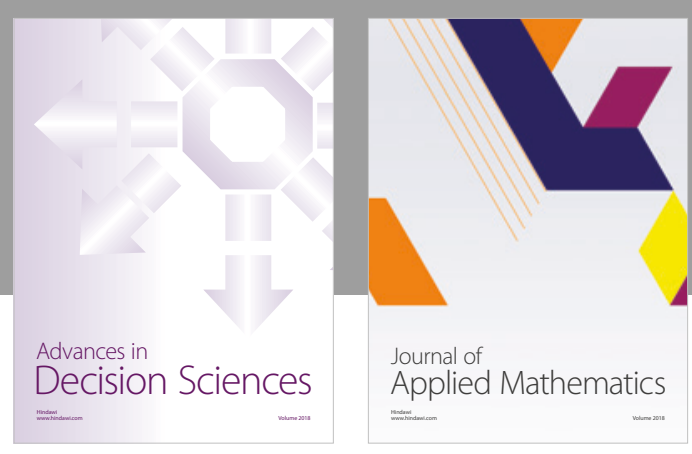

Journal of

Applied Mathematics
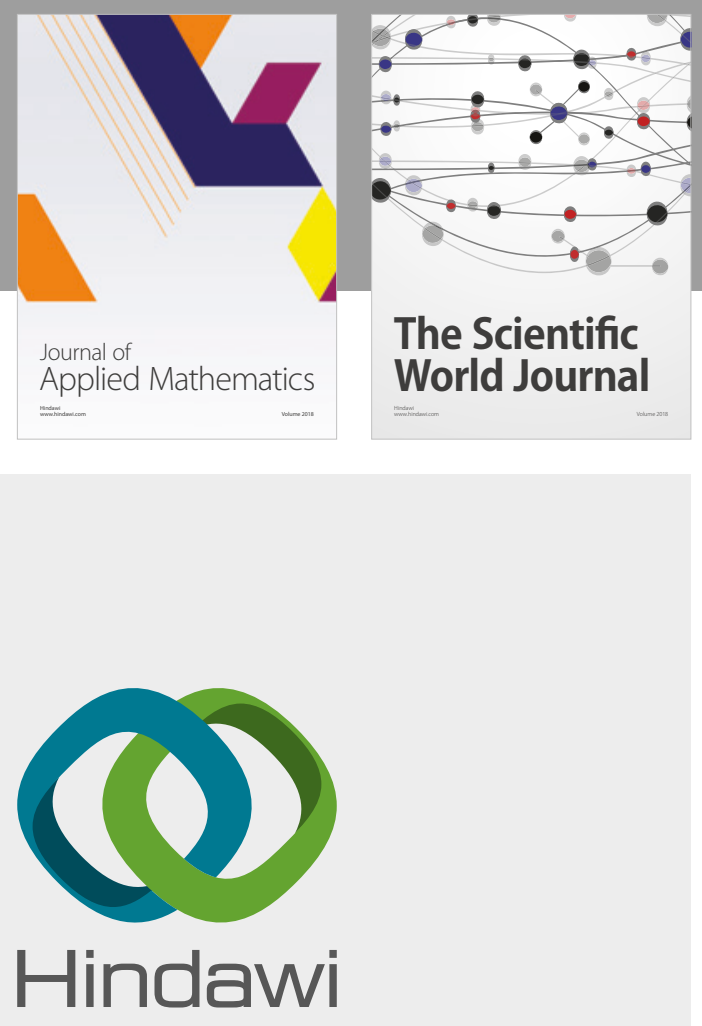

Submit your manuscripts at

www.hindawi.com

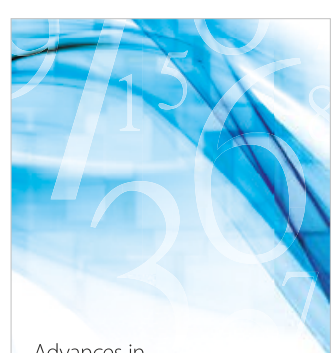

Advances in
Numerical Analysis
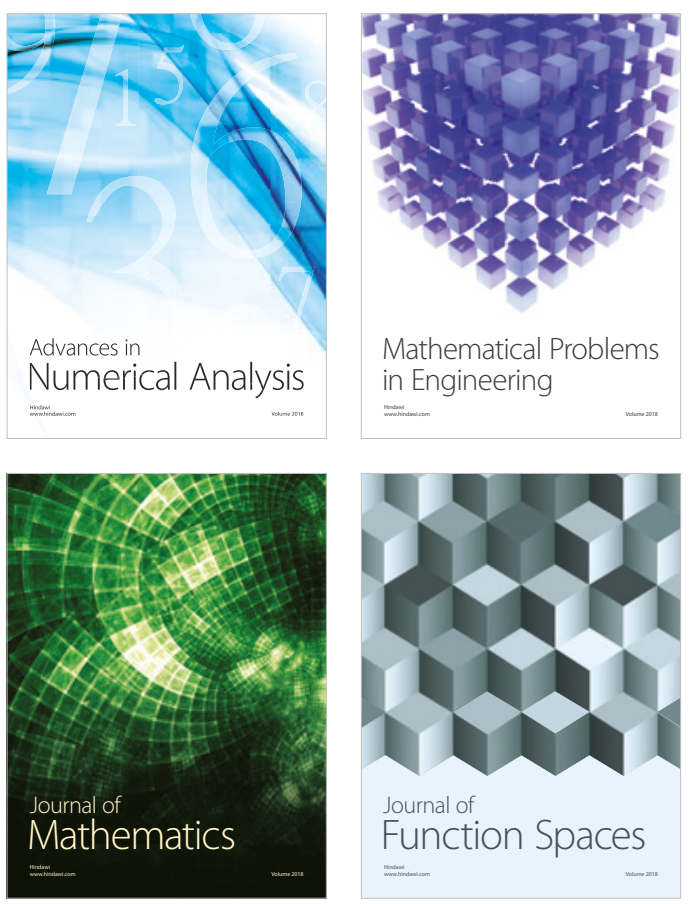

Mathematical Problems in Engineering

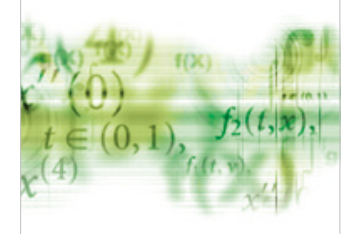

International Journal of

Differential Equations

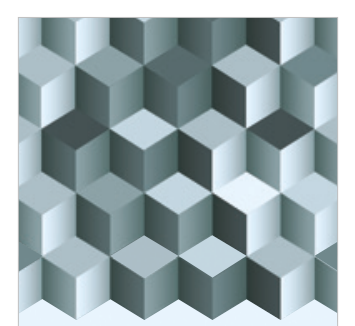

Journal of

Function Spaces
The Scientific

World Journal

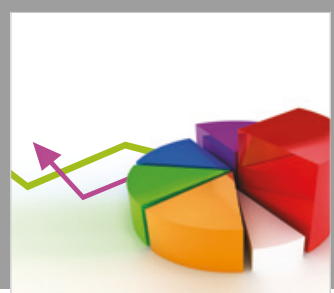

Journal of

Probability and Statistics
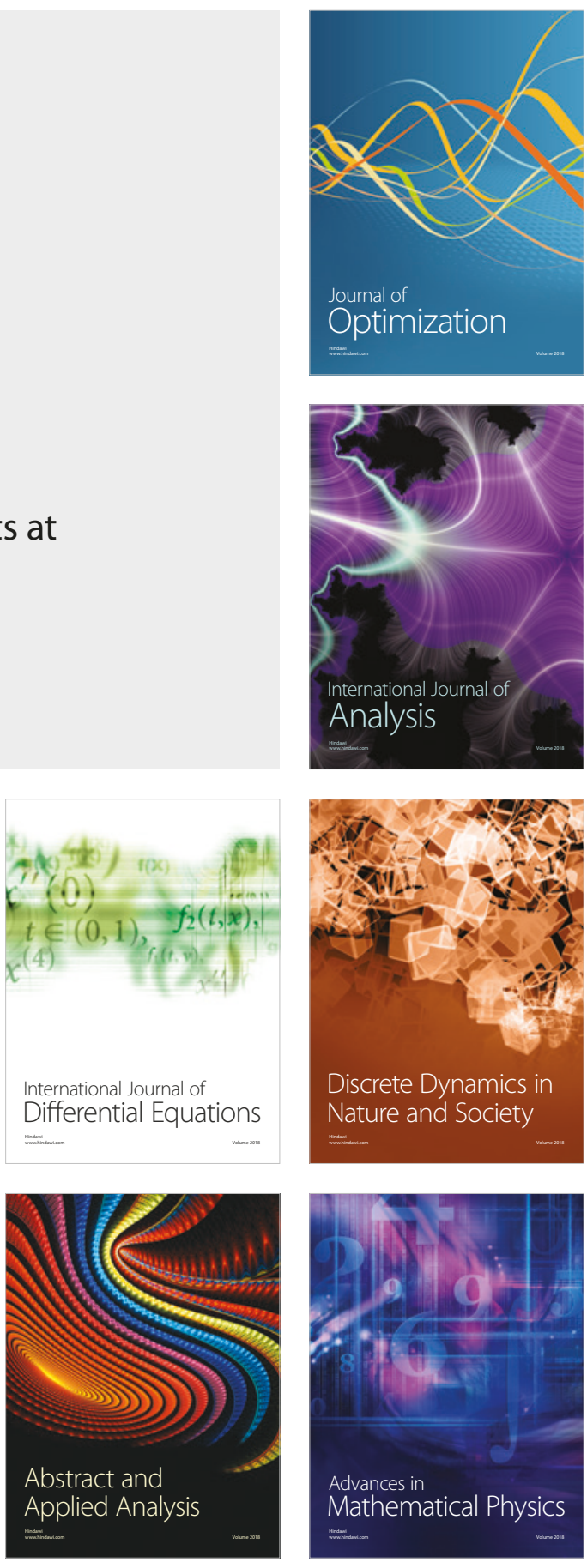